\title{
Comparison of Sunyaev-Zel'dovich measurements from Planck and from the Arcminute Microkelvin Imager for 99 galaxy clusters ${ }^{\star}$
}

\author{
Y. C. Perrott ${ }^{2}$, M. Olamaie ${ }^{2}$, C. Rumsey ${ }^{2}$, M. L. Brown ${ }^{15}$, F. Feroz ${ }^{2}$, K. J. B. Grainge ${ }^{2,16,15}$, M. P. Hobson ${ }^{2}$, \\ A. N. Lasenby 2,16 , C. J. MacTavish ${ }^{16}$, G. G. Pooley ${ }^{2}$, R. D. E. Saunders ${ }^{2,16}$, M. P. Schammel ${ }^{2,10}$, P. F. Scott ${ }^{2}$,
} T. W. Shimwell ${ }^{2,5}$, D. J. Titterington ${ }^{2}$, E. M. Waldram ${ }^{2}$, N. Aghanim ${ }^{11}$, M. Arnaud ${ }^{17}$, M. Ashdown ${ }^{16,2}$, H. Aussel ${ }^{17}$, R. Barrena ${ }^{14,8}$, I. Bikmaev ${ }^{7,1}$, H. Böhringer ${ }^{19}$, R. Burenin ${ }^{21,20}$, P. Carvalho ${ }^{12,16}$, G. Chon ${ }^{19}$, B. Comis ${ }^{18}$, H. Dahle ${ }^{13}$, J. Democles ${ }^{17}$, M. Douspis ${ }^{11}$, D. Harrison ${ }^{12,16}$, A. Hempel ${ }^{14,8,23}$, G. Hurier ${ }^{11}$, I. Khamitov ${ }^{22,7}$, R. Kneissl ${ }^{9,3}$, J. F. Macías-Pérez ${ }^{18}$, J.-B. Melin ${ }^{6}$, E. Pointecouteau ${ }^{24,4}$, G. W. Pratt ${ }^{17}$, J. A. Rubiño-Martín ${ }^{14,8}$, V. Stolyarov $2,16,21$, and D. Sutton ${ }^{12,16}$

(Affiliations can be found after the references)

Received 12 May 2014 / Accepted 11 June 2015

\begin{abstract}
We present observations and analysis of a sample of 123 galaxy clusters from the 2013 Planck catalogue of Sunyaev-Zel'dovich sources with the Arcminute Microkelvin Imager (AMI), a ground-based radio interferometer. AMI provides an independent measurement with higher angular resolution, 3 arcmin compared to the Planck beams of 5-10 arcmin. The AMI observations thus provide validation of the cluster detections, improved positional estimates, and a consistency check on the fitted size $\left(\theta_{\mathrm{s}}\right)$ and flux $\left(Y_{\mathrm{tot}}\right)$ parameters in the generalised Navarro, Frenk and White (GNFW) model. We detect 99 of the clusters. We use the AMI positional estimates to check the positional estimates and error-bars produced by the Planck algorithms PowellSnakes and MMF3. We find that $Y_{\text {tot }}$ values as measured by AMI are biased downwards with respect to the Planck constraints, especially for high Planck-S/N clusters. We perform simulations to show that this can be explained by deviation from the universal pressure profile shape used to model the clusters. We show that AMI data can constrain the $\alpha$ and $\beta$ parameters describing the shape of the profile in the GNFW model for individual clusters provided careful attention is paid to the degeneracies between parameters, but one requires information on a wider range of angular scales than are present in AMI data alone to correctly constrain all parameters simultaneously.
\end{abstract}

Key words. cosmology: observations - galaxies: clusters: general - galaxies: clusters: intracluster medium - cosmic background radiation $\mathrm{X}$-rays: galaxies: clusters

\section{Introduction}

The Planck satellite data-release of 2013 included a catalogue of 1227 galaxy clusters detected via the Sunyaev-Zel'dovich (SZ, Sunyaev \& Zel'dovich 1972) effect (Planck Collaboration XXIX 2014). This is the deepest all-sky cluster catalogue in SZ to date, consisting of clusters spanning redshifts up to $\approx 1$, and masses of around $10^{14} M_{\odot}$ to $10^{15} M_{\odot}$. SZ-selected samples have the advantage of a clean, and much less redshift-dependent (above $z \approx 0.3$ ) selection function in mass than, for example, X-rayselected samples (Planck Collaboration XX 2014); in addition, simulations predict that the SZ flux correlates more tightly with mass than, for example, X-ray or optical observable quantities (e.g. da Silva et al. 2004; Motl et al. 2005; Nagai 2006; Aghanim et al. 2009; Angulo et al. 2012; Kay et al. 2012). The Planck SZ catalogue is therefore a potentially very powerful tool for investigating the growth of structure in the Universe; clusters in the catalogue are being followed up with optical, radio and X-ray telescopes in order to provide multi-wavelength information to understand fully their properties.

The Arcminute Microkelvin Imager (AMI; Zwart et al. 2008) is a dual-array interferometer designed for SZ studies, which is situated near Cambridge, UK. AMI consists of two arrays: the Small Array (SA), optimised for viewing

* Appendices are available in electronic form at http: //www . aanda.org arcminute-scale features, having an angular resolution of $\approx 3$ arcmin and sensitivity to structures up to $\approx 10$ arcmin in scale; and the Large Array (LA), with angular resolution of $\approx 30$ arcsec, which is insensitive to the arcminute-scale emission due to clusters and is used to characterise and subtract confusing radio sources. Both arrays operate at a central frequency of $\approx 15 \mathrm{GHz}$ with a bandwidth of $\approx 4.5 \mathrm{GHz}$, divided into six channels. For further details of the instrument, see Zwart et al. (2008).

In a previous paper, (Planck and AMI Collaborations 2013, from here on AP2013) a sample of 11 clusters selected from the Planck Early Release Catalogue was followed up with AMI in order to check the consistency of the cluster parameters as measured by the two telescopes, finding the SZ signals as measured by AMI to be, on average, fainter and of smaller angular size. We have used AMI to observe all of the clusters in the Planck 2013 SZ catalogue that are at declinations easily observable with AMI (excluding those at very low redshift). This serves two purposes: (a) to investigate the discrepancies found in AP2013 further; and (b) to provide validation of, improved positional estimates for, and higher-resolution SZ maps of a large number of Planck cluster detections. We here present these observations and our analysis of them.

The paper is organised as follows. In Sect. 2 we describe the selection of the cluster sample. In Sect. 3 we describe the AMI observations and data reduction, and in Sect. 4 we outline the model used to describe the SZ signal. In Sect. 4.2 we briefly 
describe the Planck data analysis and describe in more detail the analysis of the AMI data in Sect. 4.3, including our detection criteria. Section 4.4 contains some representative examples of the results, and Sects. 4.4.6 and 4.4.7 compare the cluster parameter estimates produced by AMI to those produced by Planck. In Sect. 5 we use simulations to investigate the issue of variation from the universal model described in Sect. 4, and in Sect. 5.3 we present results from reanalysing the real data allowing the shape parameters in the model to vary. Finally, we conclude in Sect. 6.

\section{Selection of the cluster sample}

An initial selection cut of $20^{\circ} \leq \delta<87^{\circ}$ was applied to satisfy AMI's easy observing limits; although AMI can observe to lower declinations, increased interference due to geostationary satellites makes observing large samples below $\delta=20^{\circ}$ currently difficult. In addition, clusters with known redshifts of $z \leq 0.100$ were excluded since these have large angular sizes and will be largely resolved out by AMI; although the brightest of these will still be detectable, it will be difficult to constrain their properties using AMI data. These initial cuts resulted in an initial sample size of 337 with Planck signal-to-noise $(\mathrm{S} / \mathrm{N})$ values ranging from 4.5-20. In this paper, we present results for the subset of the sample with $S / N \geq 5$; this reduces the sample to 195 . Results for the remaining clusters with $4.5 \leq S / N<5$ will be released at a later date.

As in the optical, where confusion due to a bright star or a crowded field can affect the detection likelihood, a benign radio point source environment is important for AMI, but the requisite benignness is difficult to quantify. In practice, the effect of the source environment on the detection potential of a cluster depends on many factors including the number, location and orientation of the sources with respect to each other and to the sidelobes of the primary and synthesised beams. Non-trivial source environments can create complex and overlapping sidelobe patterns which can create spurious sources or reduce the flux density of real sources. In turn, the synthesised beam depends on $u v$-coverage, which changes for different $\delta$ and hour-angle coverage of observations of a given cluster. The primary beam is a function of frequency so the effect of a source at a given offset from the pointing centre also depends on its spectrum. These effects are almost impossible to quantify in a systematic way. In order to apply at least consistent criteria across the whole sample, the following criteria were applied based on LA observations: clusters were discarded if there were radio sources of peak flux density $S_{\text {peak }}>5 \mathrm{mJy}$ within $3 \mathrm{arcmin}$ of the pointing centre, of $S_{\text {peak }}>20 \mathrm{mJy}$ within $10 \mathrm{arcmin}$ of the pointing centre, or extended emission with fitted (deconvolved) major-axis size $>2$ arcmin and integrated flux density $S_{\text {int }}>2$ mJy anywhere on the map; experience suggests that observation of the SZ signal in such clusters with AMI is unreliable. Clusters were discarded for source environment based either on existing observations or, for clusters that had not been previously observed with AMI, based on a short pre-screening observation carried out with the LA. It should be noted that some clusters which have been previously observed and detected by AMI are excluded by these cuts; some of the new clusters discarded by this process may also be observable.

In addition, clusters were visually inspected at various stages of the follow-up and analysis process, and some were rejected at later stages due to extra source environment problems such as extended emission not visible on the LA map, or very bright sources just outside the LA detection radius which affect the SA
Table 1. Numbers of clusters in the $20^{\circ} \leq \delta<87^{\circ}$, Planck $S / N \geq 5$ sub-sample in various categories.

\begin{tabular}{lr}
\hline \hline Category & Number of clusters \\
\hline Total & 229 \\
$z \leq 0.100$ & 34 \\
Automatic radio-source environment rejection & 52 \\
Manual radio-source environment rejection & 20 \\
Included in sample & 123 \\
\hline
\end{tabular}

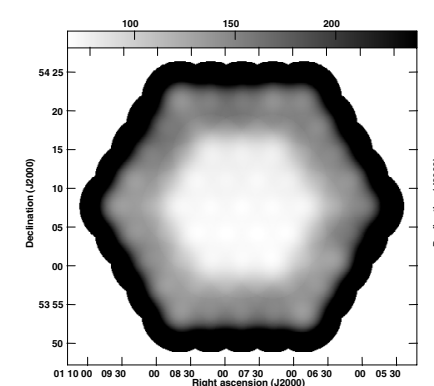

(a)

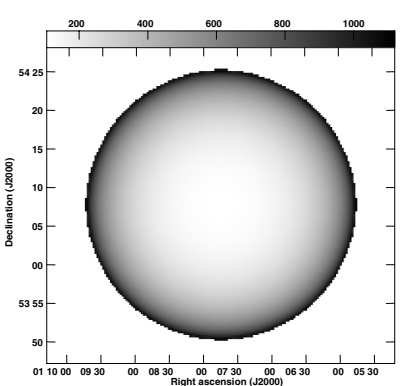

(b)
Fig. 1. Noise maps for a typical cluster observation at $\delta \approx 54^{\circ}$ on the AMI-LA a) and SA b). The grey-scales are in $\mu \mathrm{Jy}_{\text {beam }}{ }^{-1}$ and on a) the grey-scale is truncated to show the range of noise levels; $\mathbf{b}$ ) is cut off at the $10 \%$ power point of the primary beam.

map due to the larger primary beam. Here we present results for the so obtained final sub-sample, which we will refer to as the SZ sample, consisting of 123 clusters. A breakdown of the numbers of clusters rejected for various reasons is shown in Table 1.

The full list of clusters within the AMI observational bounds and their reason for rejection, if not part of the SZ sample, is given in Appendix A. In addition, as a service to the community for each cluster we provide information on the $15 \mathrm{GHz}$ radio point source environment ${ }^{1}$.

\section{Description of AMI data}

Clusters are observed using a single pointing centre on the SA, which has a primary beam of size $\approx 20$ arcmin FWHM, to noise levels of $\lesssim 120 \mu \mathrm{Jy}_{\text {beam }}{ }^{-1}$. To cover the same area with the LA, which has a primary beam of size $\approx 6$ arcmin FWHM, the cluster field is observed as a 61-point hexagonal raster. The noise level of the raster is $\lesssim 100 \mu \mathrm{Jy}_{\text {beam }}{ }^{-1}$ in the central 19 pointings, and slightly higher in the outer regions. Typical noise maps and $u v$-coverages are displayed for both arrays in Figs. 1 and 2 . The average observation time for a cluster is $\approx 30 \mathrm{~h}$ on both arrays.

Data on both arrays are flagged for interference and calibrated using the AMI in-house software package REDUCE. Flux calibration is applied using contemporaneous observations of the primary calibration sources $3 \mathrm{C} 286,3 \mathrm{C} 48$, and $3 \mathrm{C} 147$. The assumed flux densities for 3C 286 were converted from Very Large Array total-intensity measurements (Perley \& Butler 2013), and are consistent with the Rudy et al. (1987) model of Mars transferred on to absolute scale, using results from the Wilkinson Microwave Anisotropy Probe. The assumed flux densities for 3C 48 and 3C 147 are based on long-term monitoring with the SA using 3C 286 for flux calibration (see Table 2). Phase calibration is applied using interleaved observations of a nearby bright

\footnotetext{
1 Available online at http://wWw.astro.phy.cam.ac.uk/ surveys/ami-planck/
} 


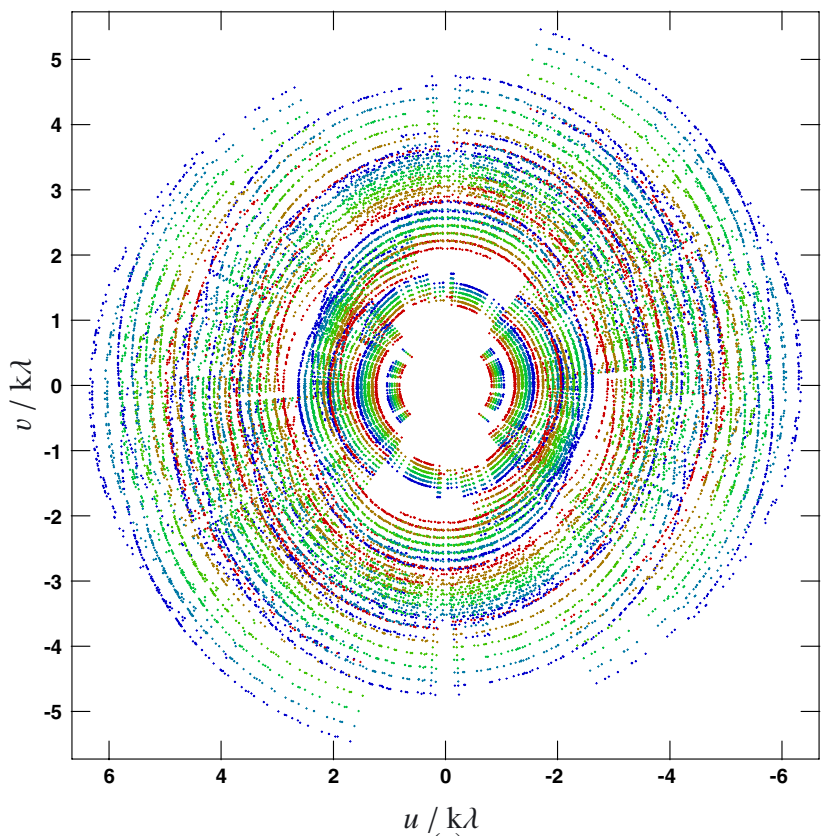

(a)

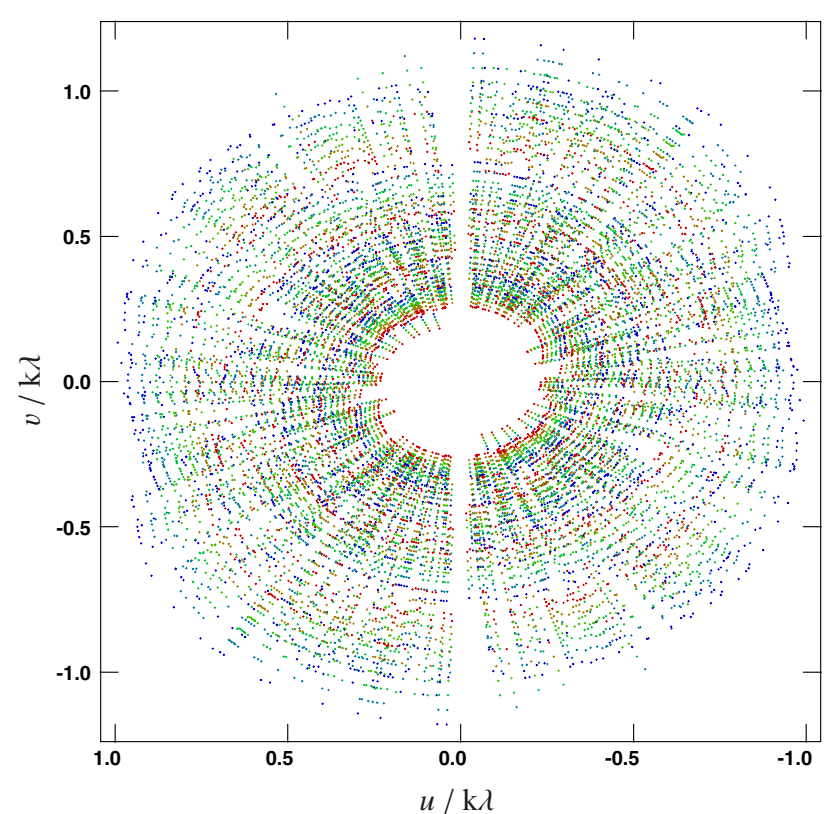

(b)

Fig. 2. $u v$-coverages for a typical cluster observation at $\delta \approx 54^{\circ}$, for the AMI-LA a) and SA b). The colours indicate different channels. Note the different axis scales; the short baselines of the SA are designed for sensitivity to arcminute-scale cluster emission, while the longer baselines of the LA are insensitive to emission on this scale and are used to characterise and subtract the foreground radio sources.

Table 2. Assumed $I+Q$ flux densities of 3C 286, 3C 48 and 3C 147.

\begin{tabular}{ccccc}
\hline \hline Channel & $\bar{v} / \mathrm{GHz}$ & $S^{3 \mathrm{C} 286} / \mathrm{Jy}$ & $S^{3 \mathrm{C} 48} / \mathrm{Jy}$ & $S^{3 \mathrm{C} 147} / \mathrm{Jy}$ \\
\hline 3 & 13.88 & 3.74 & 1.89 & 2.72 \\
4 & 14.63 & 3.60 & 1.78 & 2.58 \\
5 & 15.38 & 3.47 & 1.68 & 2.45 \\
6 & 16.13 & 3.35 & 1.60 & 2.34 \\
7 & 16.88 & 3.24 & 1.52 & 2.23 \\
8 & 17.63 & 3.14 & 1.45 & 2.13 \\
\hline
\end{tabular}

source selected from the VLBA Calibrator survey (Petrov et al. 2008); in the case of the LA, a secondary amplitude calibration is also applied using contemporaneous observations of the phasecalibration source on the SA.

Maps of the SA and LA data are made using AIPS $^{2}$, CLEANing in an automated manner. Source-finding is carried out at $4 \sigma$ on the LA continuum map, as described in Davies et al. (2011) and Franzen et al. (2011), and sources that are detected at $\geq 3 \sigma$ on at least three channel maps and are not extended have a spectral index $\alpha$ fitted across the AMI band. SA data are binned on a grid in $u v$-space in order to reduce the memory required for subsequent analysis.

\section{Analysing the SZ signal}

\subsection{Cluster model}

For consistency with the Planck catalogue, in this paper we assume the electron pressure profile $P_{\mathrm{e}}(r)$ of each cluster follows a generalised Navarro-Frenk-White (GNFW, Navarro et al. 1997) model, which is given by (assuming spherical geometry)

$P_{\mathrm{e}}(r)=P_{0}\left(\frac{r}{r_{\mathrm{s}}}\right)^{-\gamma}\left[1+\left(\frac{r}{r_{\mathrm{s}}}\right)^{\alpha}\right]^{(\gamma-\beta) / \alpha}$,

\footnotetext{
2 http://aips.nrao.edu/
}

where $P_{0}$ is a normalisation coefficient, $r$ is the physical radius, $r_{\mathrm{s}}$ is a characteristic scale radius, and the parameters $(\gamma, \alpha, \beta)$ describe the slopes of the pressure profile at radii $r \ll r_{\mathrm{s}}, r \approx r_{\mathrm{s}}$, and $r \gg r_{\mathrm{s}}$ respectively (Nagai et al. 2007). Following Arnaud et al. (2010), we fix the slope parameters to their universal values, $\gamma=0.3081, \alpha=1.0510, \beta=5.4905$ derived from the REXCESS sample (Böhringer et al. 2007). They are also fixed to these values in the Planck analysis.

Given this model, the integrated SZ surface brightness, or integrated Compton- $y$ parameter, for a cluster is given by

$Y_{\mathrm{sph}}(r)=\frac{\sigma_{\mathrm{T}}}{m_{\mathrm{e}} c^{2}} \int_{0}^{r} P_{\mathrm{e}}\left(r^{\prime}\right) 4 \pi r^{\prime 2} \mathrm{~d} r^{\prime}$,

where $\sigma_{\mathrm{T}}$ is the Thomson scattering cross-section, $m_{\mathrm{e}}$ is the electron mass, and $c$ is the speed of light. This has an analytical solution as $r \rightarrow \infty$, giving the total integrated Compton- $y$ parameter $Y_{\text {tot,phys }}$ as

$Y_{\text {tot,phys }}=\frac{4 \pi \sigma_{\mathrm{T}}}{m_{\mathrm{e}} c^{2}} P_{0} r_{\mathrm{s}}^{3} \frac{\Gamma\left(\frac{3-\gamma}{\alpha}\right) \Gamma\left(\frac{\beta-3}{\alpha}\right)}{\alpha \Gamma\left(\frac{\beta-\gamma}{\alpha}\right)}$.

With $(\gamma, \alpha, \beta)$ fixed, a cluster's appearance on the sky may be described using four (observational) parameters only: $\left(x_{0}, y_{0}, \theta_{\mathrm{s}}, Y_{\mathrm{tot}}\right)$, where $x_{0}$ and $y_{0}$ are the positional coordinates for the cluster, $\theta_{\mathrm{s}}=r_{\mathrm{s}} / D_{\mathrm{A}}$ is the characteristic angular scale of the cluster on the sky $\left(D_{\mathrm{A}}\right.$ is the angular diameter distance to the cluster), and $Y_{\text {tot }}=Y_{\text {tot,phys }} / D_{\mathrm{A}}^{2}$ is the SZ surface brightness integrated over the cluster's extent on the sky.

This model does not require any redshift information; physical quantities such as $r_{\mathrm{s}}$ and $Y_{\text {tot,phys }}$ can be recovered from $\theta_{\mathrm{s}}$ and $Y_{\text {tot }}$ given a redshift. Alternatively, $r_{X}$ and $M_{X}$ for some overdensity radius $X$ can be recovered given a redshift, a concentration parameter $c_{X} \equiv r_{X} / r_{\mathrm{s}}$ and some model or scaling relationship for translating $Y$ into mass (e.g. Planck Collaboration XX 2014; Olamaie et al. 2012). Physical modelling will not be addressed in this paper. 
Note that in the Planck analysis, in order to impose a finite integration extent, $Y_{5 R_{500}}$ (the SZ surface brightness integrated to $\left.5 \times R_{500}\right)$ is estimated rather than $Y_{\text {tot }}$. For the universal GNFW parameter values, (with $c_{500}=1.177$ ), the two quantities are equivalent to within $5 \%$.

\subsection{Analysis of Planck data}

The Planck SZ catalogue is the union of the catalogues produced by three detection algorithms: MMF1 and MMF3, which are multi-frequency matched-filter detection methods, and PowellSnakes (PwS), which is a Bayesian detection method. Full details of these algorithms are provided in Melin et al. (2006), Carvalho et al. (2009, 2012) and Melin et al. (2012). Since the PwS analysis methodology most closely matches the Bayesian analysis procedures used to analyse AMI data, we take cluster parameters produced by PwS as our preferred Planck values, followed by MMF3, and finally MMF1 values where a particular cluster is not detected by all algorithms.

\subsection{Analysis of AMI data}

The model attempting to describe the AMI data is produced by a combination of the cluster model described above, the radio source environment as measured by the LA and a generalised Gaussian noise component comprising instrumental noise, confusion noise from radio sources below the detection threshold, and contamination from primordial CMB anisotropies.

Each foreground radio source is modelled by the parameters $\left(x_{S}, y_{S}, S_{0}, \alpha\right)$. Positions $\left(x_{S}, y_{S}\right)$ and initial estimates of the flux density at a central frequency $\left(S_{0}\right)$ are produced from the LA channel-averaged maps; for sources detected at $\geq 3 \sigma$ on at least three of the individual channel maps, a spectral index $\alpha$ is also fitted to the channel flux densities. The flux density and spectral index of sources which are detected at $\geq 4 \sigma$ on the SA map are modelled simultaneously with the cluster; this accounts for possible source variability (although we attempt to observe clusters close in time on the two arrays, this is not always possible due to different demands on the observing time of the arrays) and inter-array calibration uncertainty. Flux densities are given a Gaussian prior with $\sigma=40 \%$; where $\alpha$ has been fitted from the LA data, a Gaussian prior with width corresponding to the fitting uncertainty is applied, otherwise a prior based on the $10 \mathrm{C}$ survey is applied (Davies et al. 2011). Sources detected at $<4 \sigma$ on the SA map are subtracted directly based on the LA values of $S_{0}$ and $\alpha$ (or the median of the 10C prior where $\alpha$ has not been fitted) initially. If the cluster position output from the analysis has directly-subtracted sources within 3 arcmin, the analysis is repeated with those sources also modelled. The positions of the sources are always fixed to their LA values as the LA has higher positional precision.

In the cluster model, $x_{0}$ and $y_{0}$ are the offsets in RA and $\delta$ from the pointing centre of the SA observation; for previouslyknown clusters with existing AMI data, the pointing centre is the X-ray position of the cluster, while for new clusters it is the Planck position. Gaussian priors are used on $x_{0}$ and $y_{0}$, centred on the Planck position (i.e. offset from the pointing/phase reference centre, if the pointing centre is the X-ray position) and with width given by the Planck positional uncertainty up to a maximum of 5 arcmin; larger priors allow the detection algorithm to fix on noise features toward the edges of the SA primary beam, which has a FWHM of $\approx 20$ arcmin. In practice, no PwS positional errors in the sample are greater than 5 arcmin. MMF1 does not give positional error estimates, so clusters detected only by MMF1 are given the maximum 5 arcmin error; some clusters detected by MMF3 (but not PwS) have positional errors $>5$ arcmin, but as will be shown in Sect. 4.4.6, MMF3 positional errors tend to be over-estimated.

Model parameter estimation is performed in a fully Bayesian manner using the AMI in-house software package MCADAM, in $u v$-space (see, e.g. Feroz et al. 2009b for more details). Bayes' theorem states that

$\operatorname{Pr}(\boldsymbol{\Theta} \mid \mathbf{D}, H)=\frac{\operatorname{Pr}(\mathbf{D} \mid \boldsymbol{\Theta}, H) \operatorname{Pr}(\boldsymbol{\Theta} \mid H)}{\operatorname{Pr}(\mathbf{D} \mid H)}$,

where $\boldsymbol{\Theta}$ is a set of parameters for a model, $H$, and $\mathbf{D}$ is the data. Thus, the posterior probability distribution, $\operatorname{Pr}(\Theta \mid \mathbf{D}, H)$, is proportional to the likelihood, $\operatorname{Pr}(\mathbf{D} \mid \boldsymbol{\Theta}, H)$, multiplied by the prior, $\operatorname{Pr}(\boldsymbol{\Theta} \mid H)$. The normalising factor is the evidence, $\operatorname{Pr}(\mathbf{D} \mid H) \equiv$ $\mathcal{Z}$. MCADAM uses the nested sampler MULTINEST (Feroz \& Hobson 2008; Feroz et al. 2009a) to obtain the posterior distribution for all parameters, which can be marginalised to provide two- and one-dimensional parameter constraints.

MULTINEST also calculates the evidence, which can be ignored for parameter estimation but is important for model selection, since it represents the probability of the data given a model and a prior, marginalised over the the model's parameter space:

$\mathcal{Z}=\int \operatorname{Pr}(\mathbf{D} \mid \boldsymbol{\Theta}, H) \operatorname{Pr}(\boldsymbol{\Theta} \mid H) d^{D} \boldsymbol{\Theta}$,

where $D$ is the dimensionality of the parameter space. The probability of two different models given the data can be compared using their evidence ratio:

$\frac{\operatorname{Pr}\left(H_{1} \mid \mathbf{D}\right)}{\operatorname{Pr}\left(H_{0} \mid \mathbf{D}\right)}=\frac{\operatorname{Pr}\left(\mathbf{D} \mid H_{1}\right) \operatorname{Pr}\left(H_{1}\right)}{\operatorname{Pr}\left(\mathbf{D} \mid H_{0}\right) \operatorname{Pr}\left(H_{0}\right)}=\frac{\mathcal{Z}_{1}}{\mathcal{Z}_{0}} \frac{\operatorname{Pr}\left(H_{1}\right)}{\operatorname{Pr}\left(H_{0}\right)}$,

where $\operatorname{Pr}\left(H_{1}\right) / \operatorname{Pr}\left(H_{0}\right)$ is the a priori probability ratio for the two models. To assess the detection significance of a cluster, we therefore perform two parameter estimation runs - one with the full cluster + radio source environment model $\left(H_{1}\right)$, and one with only the radio source environment model (the null run, $H_{0}$ ). We set $\operatorname{Pr}\left(H_{1}\right) / \operatorname{Pr}\left(H_{0}\right)=1$ so that $\mathcal{Z}_{1} / \mathcal{Z}_{0}$ is a measure of the detection significance for the cluster. This ratio takes into account the various sources of noise as well as the goodness of fit of the radio source and cluster models.

Figure 3 shows the distribution of $\Delta \ln (\mathcal{Z})$ values in the SZ sample. It is also useful to define discrete detection and nondetection categories based on the continuous evidence ratio values. We follow Jeffreys $(1961)$ in taking $\Delta \ln (\mathcal{Z})=0$ as the boundary between detections and non-detections. We also define an additional boundary $\Delta \ln (\mathcal{Z})=3$ between moderate and clear detections, where moderate detections are cases where the data are more consistent with the presence of a cluster than not, but there is not enough information in the data to constrain the model parameters well. For symmetry, we also define a boundary at $\Delta \ln (\mathcal{Z})=-3$ to indicate cases where the cluster model is strongly rejected by the data. These boundaries were chosen empirically, by inspecting final maps and posterior distributions. The four categories are listed in Table 3.

\subsubsection{Prior on $Y_{\text {tot }}$ and $\theta_{\mathrm{s}}$}

The priors assigned to $Y_{\text {tot }}$ and $\theta_{\mathrm{s}}$ in AP2013 and used for the Planck PWS analysis are based on marginalised distributions of $Y_{\text {tot }}$ and $\theta_{\mathrm{s}}$ in a simulated population of clusters generated according to the Jenkins mass function (Jenkins et al. 2001), as 


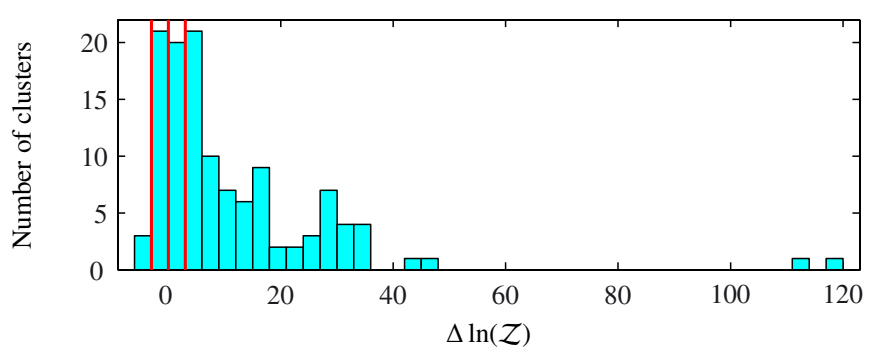

Fig. 3. Distribution of evidence ratio values in the SZ sample, with the division into detection categories given in Table 3 indicated by red vertical lines.

Table 3. Evidence difference $(\Delta \ln (\mathcal{Z}))$ boundaries used for categorising clusters as clear detections, moderate detections, non-detections and clear non-detections, and the number of clusters in each category in the SZ sample.

\begin{tabular}{lcr}
\hline \hline Category & $\Delta \ln (\mathcal{Z})$ boundaries & Number \\
\hline Clear detection $(\mathrm{Y})$ & $\Delta \ln (\mathcal{Z}) \geq 3$ & 79 \\
Moderate detection $(\mathrm{M})$ & $0 \leq \Delta \ln (\mathcal{Z})<3$ & 20 \\
Non-detection $(\mathrm{N})$ & $-3 \leq \Delta \ln (\mathcal{Z})<0$ & 21 \\
Clear non-detection $(\mathrm{NN})$ & $\Delta \ln (\mathcal{Z})<-3$ & 3 \\
\hline
\end{tabular}

described in Carvalho et al. (2012). The parameterisation functions for these priors are listed in Table 4. These priors ignore, however, the correlation between $Y_{\text {tot }}$ and $\theta_{\mathrm{s}}$; in addition, they take into account the Planck selection function only in assuming minimum and maximum cutoffs in each parameter.

To produce a better approximation to the true distribution of clusters expected to be detected by Planck, we used the results of the Planck completeness simulation (Planck Collaboration XXIX 2014, Sects. 3.1 and 3.2, Fig. 9). This simulation was produced by drawing a cluster population from the Tinker mass function (Tinker et al. 2008), and converting the redshifts and masses to $Y_{500}$ and $\theta_{500}$ observable quantities using the scaling relations in Planck Collaboration X (2011). This cluster population was injected into the real Planck data assuming GNFW pressure profiles with the shape parameters varying according to results from Planck Collaboration Int. V (2013) and a simulated union catalogue was created by running the Planck detection pipelines on the simulated dataset in the usual manner; see Planck Collaboration XXIX (2014) for more details.

We noted that the resulting two-dimensional distribution in $\theta_{\mathrm{s}}$ and $Y_{\text {tot }}$ in log-space was elliptical in shape with roughly Gaussian distribution along the principal axes and performed a two-dimensional Gaussian fit to the distribution, parameterised by width and offset in $x=\log _{10}\left(\theta_{\mathrm{s}}\right)$, width and offset in $y=$ $\log _{10}\left(Y_{\text {tot }}\right)$, and angle $\phi$ measured clockwise from the $y$-axis. The best-fit parameters are listed in Table 4, and the fit and residuals with respect to the simulated population are shown in Fig. 4. We use this fit to the simulated population as our prior on $\theta_{\mathrm{s}}$ and $Y_{\text {tot }}$.

\subsection{Results}

In the SZ sample, 79 are clear detections, 20 are moderate detections, 21 are non-detections and 3 are clear non-detections. A summary of the results for each cluster in the sample is presented in Appendix A.

Some representative examples from each category are discussed in the following. In each case, two

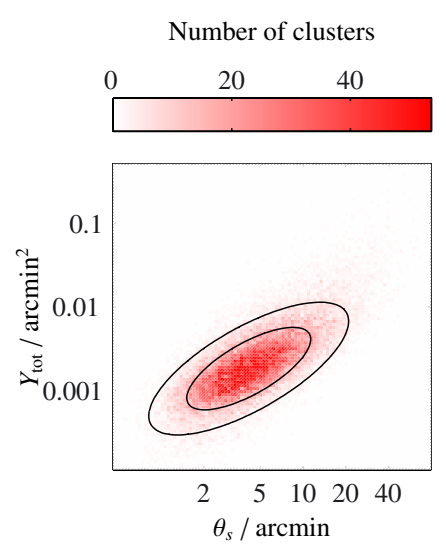

(a)

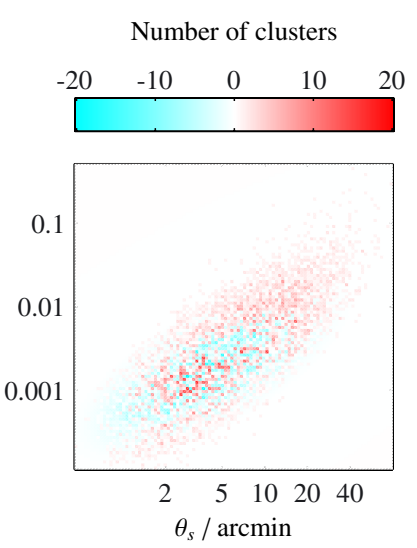

(b)
Fig. 4. a) Shows the sampled distribution (red histogram), and the two-dimensional elliptical Gaussian fit to the $Y_{\text {tot }}$ vs. $\theta_{\mathrm{s}}$ distribution in log-space (black lines, enclosing $68 \%$ and $95 \%$ of the probability). b) Shows the residuals with respect to the simulated distribution. Note that the colour-axis scales are different.

foreground-source-subtracted maps are shown; both are produced using natural weighting, and the second also has a Gaussian weighting function with the $30 \%$ point at $600 \lambda$ applied (the $u v$-tapered map). This taper downweights the longer baselines, which are only sensitive to small-angularscale features, making the extended cluster more visible. The symbols $\times$ and + show the positions of subtracted sources, respectively either modelled in MCADAM or directly subtracted based on LA values. $\square$ shows the AMI (MCADAM -determined) position of the cluster, and the $1 \times \sigma_{\text {Planck }}$ positional error radius is shown as a circle. Contours are plotted at $\pm(2,3,4, \ldots, 10) \times$ the rms noise level (measured using the AIPS task IMEAN), and dashed contours are negative. The synthesised beam is shown in the bottom left-hand corner. We emphasise that these maps are only shown for visual inspection and to assess the residual foreground contamination; all parameter estimation is done in $u v$-space.

Posterior distributions for position offset, cluster model parameters and the flux densities of the closest radio sources to the cluster centre are also shown; in these plots the units are arcsec on the sky for offset in RA $\left(x_{0}\right)$ and $\delta\left(y_{0}\right), \operatorname{arcmin}^{2}$ for $Y_{\text {tot }}$, arcmin for $\theta_{\mathrm{s}}$ and $\mathrm{mJy}$ for radio source flux densities. The blue (pink) areas correspond to regions of higher (lower) probability density. The $Y_{\text {tot }}-\theta_{\mathrm{s}}$ posterior distribution is shown separately with solid black lines for the AMI constraints overlaid with that obtained by PWS using Planck data for the cluster in red, as well as the AMI prior (black dashed lines). The joint constraint is shown in yellow where appropriate. In all cases, the contours mark the $68 \%$ and $95 \%$ confidence limits in the posterior or prior probability distributions. Similar maps and posterior distribution plots for the entire sample are available online ${ }^{3}$.

\subsubsection{Clear detections}

Abell 2218 (PSZ1 G097.72+38.13)

Abell 2218 (Abell 1958) is an extremely well-known cluster and one of the earliest SZ detections (e.g. Birkinshaw et al. 1978, 1984; Jones et al. 1993). It lies at redshift $z=0.171$ (Kristian et al. 1978). It has been observed by AMI previously as part of the LoCuSS sample (Rodríguez-Gonzálvez et al. 2012) and

3 http://www.astro.phy.cam.ac.uk/surveys/ami-planck/ 
Table 4. Priors used on profile fit parameters.

\begin{tabular}{lccc}
\hline \hline Parameter & Prior type & Parameters & Limits \\
\hline$x_{0}, y_{0}$ & Gaussian, $\mathrm{e}^{-x^{2} / 2 \sigma^{2}}$ & $\sigma=\min \left(5\right.$ arcmin, $\left.\sigma_{\text {Planck }}\right)$ & - \\
$Y_{\text {tot }}(\mathrm{old})$ & Power-law, $x^{-a}$ & $a=1.6$ & $0.0005<x<0.2$ \\
$\theta_{\mathrm{s}}$ (old) & Exponential, $\lambda \mathrm{e}^{-\lambda x}$ & $\lambda=0.2$ & $1.3<x<45$ \\
& 2D elliptical Gaussian & $x_{0}=0.6171, \sigma_{x}=0.1153$, & \\
$Y_{\text {tot }}, \theta_{\mathrm{s}}$ (new) $)$ & in $x=\log _{10}\left(\theta_{\mathrm{s}}\right)$, & $y_{0}=-2.743, \sigma_{y}=0.2856$, & $1.3<\theta_{\mathrm{s}}$ \\
& $y=\log _{10}\left(Y_{\text {tot }}\right)$ & $\phi=40.17^{\circ}$ & \\
\hline
\end{tabular}

was also in AP2013. It has the highest Planck S/N in the final subsample and is also well-detected by AMI with $\Delta \ln (\mathcal{Z})=34$. Figure 5 shows that the cluster is resolved by AMI as the depth of the decrement increases in the $u v$-tapered map, and structure can be clearly seen in the naturally-weighted map. The posterior distributions (Fig. 6) show good constraints in both position and the cluster model parameters. The two-dimensional posterior distributions for the flux densities of the three most significant nearby sources are included in the plot; it can be seen that there is some correlation between the flux densities of the sources and $Y_{\text {tot }}$, i.e. lower values of the flux densities allow lower values of $Y_{\text {tot }}$, but this does not affect the parameter constraints significantly. There is also some correlation between the flux densities of the sources and the cluster position. The remaining two sources near the cluster centre are fainter and were not modelled in the initial analysis since they appear at $<4 \sigma$ on the SA map; there is no evidence for degeneracy between the flux densities of these sources and the cluster parameters. As in AP2013 (see their Fig. 5), the PWS $Y_{\text {tot }}-\theta_{\text {s }}$ posterior overlaps with the AMI posterior, but AMI finds the cluster to be smaller and fainter than Planck (at low significance for this particular cluster).

$Y_{\text {tot }}$ is the total SZ signal of the cluster and corresponds to the zero-spacing flux, which is not measured by an interferometer; the constraints produced by AMI on $Y_{\text {tot }}$ therefore rely on extrapolating the signal on the angular scales that AMI does measure ( $\approx 200$ to $1200 \lambda$, corresponding to $\approx 15$ to $3 \mathrm{arcmin})$ to $0 \lambda$ assuming a fixed profile. Since this is a relatively nearby, large-angular-size cluster (i.e. $\theta_{500}$ inferred from the X-ray luminosity is 6.4 arcmin (Böhringer et al. 2000; Piffaretti et al. 2011) corresponding to $\theta_{\mathrm{s}}=5.4 \mathrm{arcmin}$ for the universal value of $c_{500}=1.177$, in agreement with the AMI constraint and slightly smaller than the preferred Planck value), much of the flux of the cluster exists on scales that are not measured by AMI. $Y_{\text {tot }}$ is therefore not well constrained and the $Y_{\text {tot }}-\theta_{\mathrm{s}}$ degeneracy is large compared to that produced by Planck, which measures $Y_{\text {tot }}$ directly. Nonetheless, the different degeneracy direction means that combining the two posteriors results in a tighter constraint (assuming no systematic difference between the two instruments, which will be discussed in Sect. 4.4.7).

\section{PSZ1 G060.12+11.42}

This is a new, previously unconfirmed (at the time the catalogue was published) cluster discovered by Planck at high $\mathrm{S} / \mathrm{N}$ (7.2) and clearly detected by AMI with $\Delta \ln (\mathcal{Z})=16$. The sourcesubtracted maps for the cluster are shown in Fig. 7, and the posterior distributions in Fig. 8. Again, it is clear that AMI resolves the cluster. The source flux densities of the two nearest sources are shown in the posterior distributions; there is no apparent degeneracy between the source flux densities and any of the parameters. In this case, the posterior distributions for $\theta_{\mathrm{s}}$ and $Y_{\text {tot }}$ are very consistent with the PWS posteriors. The AMI and

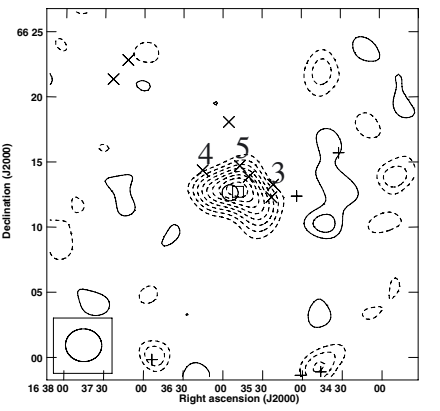

(a)

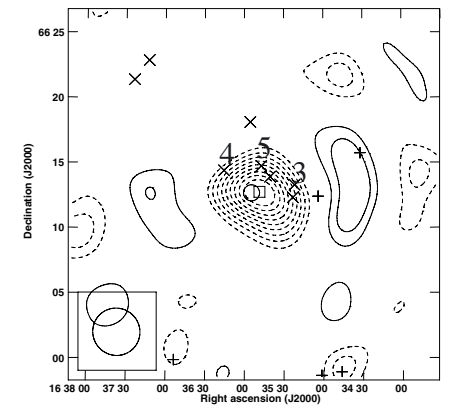

(b)
Fig. 5. SA source-subtracted map of A2218 with a) natural weighting and b) a $u v$-taper. The r.m.s. noise levels are 131 and $163 \mu \mathrm{Jy}_{\text {beam }}^{-1}$ respectively. The numbered sources have posterior distributions for their flux densities plotted in Fig. 6. See Sect. 4.4 for more details on the plots.

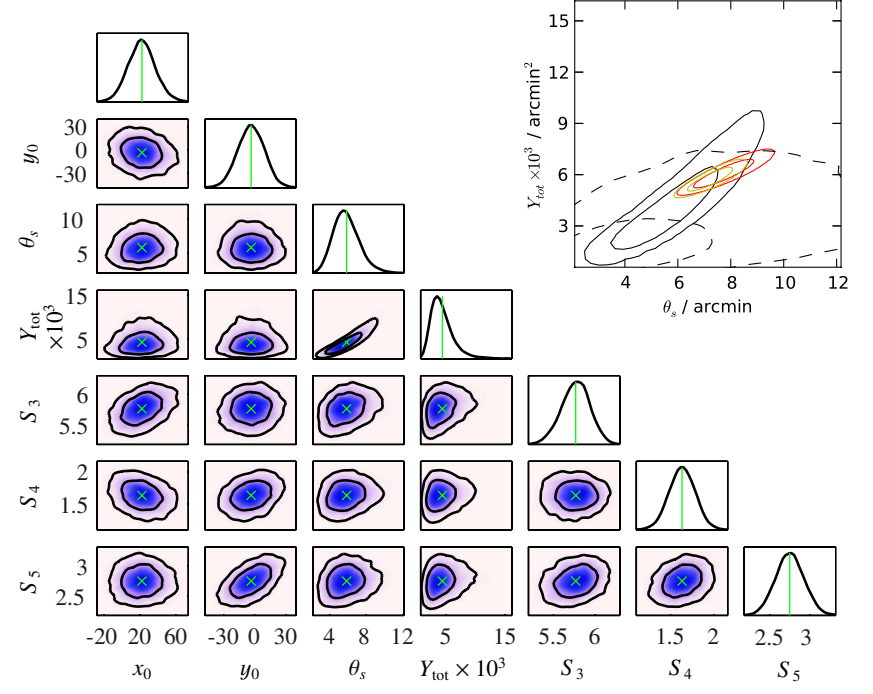

Fig. 6. AMI posterior distributions for A2218 and the $Y_{\text {tot }}-\theta_{\mathrm{s}}$ posterior overlaid with that obtained by Planck in red, and the prior as a black dotted line (upper right-hand corner). The joint constraint is shown in yellow. See Sect. 4.4 for more details on the plots.

PwS degeneracies are in different directions, meaning that the joint constraints produced by combining the two are considerably tighter.

\subsubsection{Moderate detections}

\section{ZW8503 (PSZ1 G072.78-18.70)}

ZW8503 is a well-known cluster at $z=0.143$ (Allen et al. 1992) with a large angular size $\left(\theta_{\mathrm{s}} \approx 8\right.$ arcmin as measured by Planck); 
Y. C. Perrott et al.: Planck and AMI SZ measurements for 99 galaxy clusters

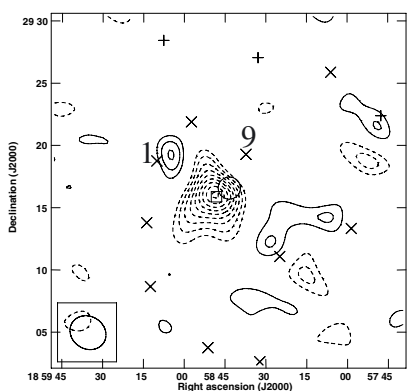

(a)

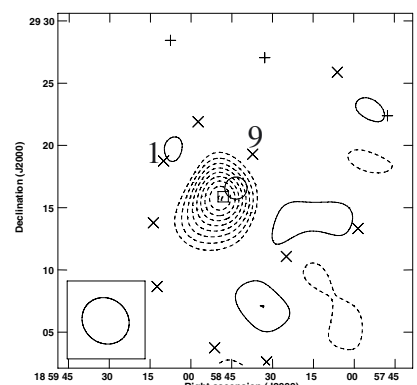

(b)
Fig. 7. SA source-subtracted map of PSZ1 G060.12+11.42 with a) natural weighting and b) a $u v$-taper. The rms noise levels are 96 and $131 \mu \mathrm{Jy}$ beam $^{-1}$ respectively. The numbered sources have posterior distributions for their flux densities plotted in Fig. 8. See Sect. 4.4 for more details on the plots.
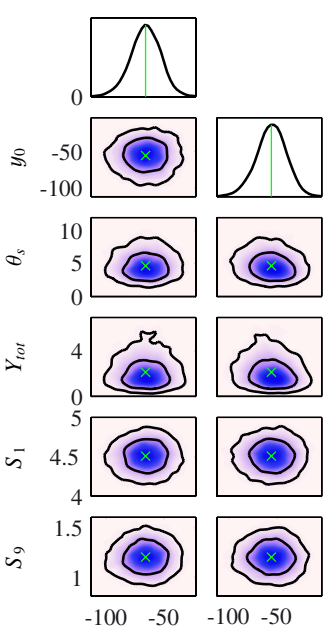

$x_{0}$
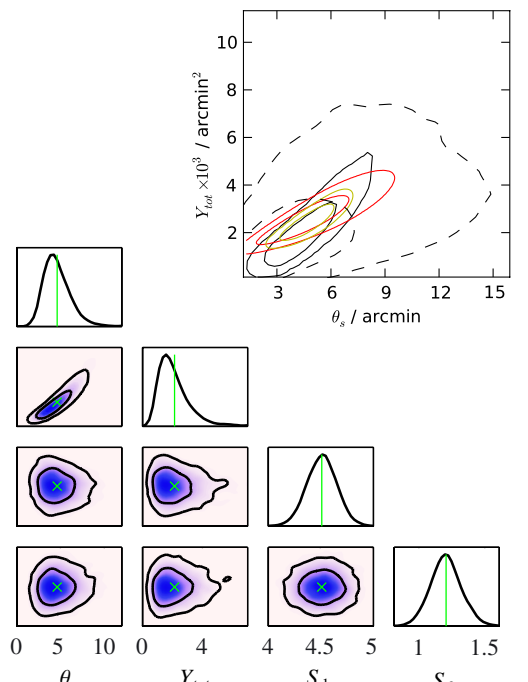

Fig. 8. AMI posterior distributions for PSZ1 G060.12+11.42 and the $Y_{\text {tot }}-\theta_{\mathrm{s}}$ posterior overlaid with that obtained by Planck (upper right-hand corner). The joint constraint is shown in yellow. See Sect. 4.4 for more details on the plots.

it is therefore not too surprising that AMI does not detect it well. A decrement at the phase centre is visible in the sourcesubtracted maps (Fig. 9), and a model with a cluster is favoured over one without by $\Delta \ln (\mathcal{Z})=1.8$, but Fig. 10 shows that there is not enough information in the AMI data to constrain the cluster parameters well, and the $Y_{\mathrm{tot}}-\theta_{\mathrm{s}}$ posterior distribution is strongly influenced by the prior (plotted as a black dotted line for comparison). There is also significant degeneracy between the cluster parameters $\left(x_{0}, y_{0}, \theta_{\mathrm{s}}, Y_{\mathrm{tot}}\right)$ and the flux densities of the closest sources. The parameter space indicated by the Planck posterior is completely ruled out by the AMI posterior distribution. The AMI map shows a good positional coincidence with the X-ray emission (Fig. 11) and also shows some substructure within the cluster; if this is real, the spherical cluster model with the universal pressure profile (derived from fits to relaxed clusters) may not provide a good fit and the extrapolated $Y_{\text {tot }}$ result may be biased.

\footnotetext{
Courtesy of the Chandra X-ray Observatory Center and the Chandra Data Archive, http://cxc.cfa.harvard.edu/cda/ (ivo://ADS/Sa.CXO\#obs/13379)
}

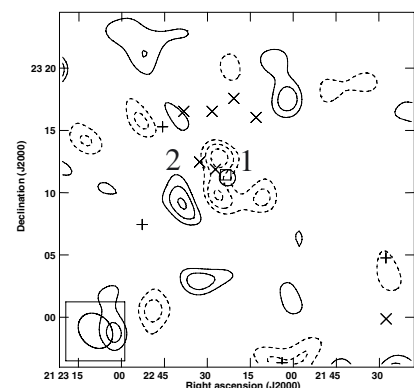

(a)

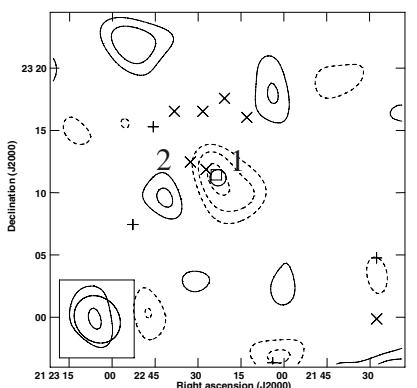

(b)
Fig. 9. SA source-subtracted map of ZW8503 with a) natural weighting and b) a $u v$-taper. The rms noise levels are 90 and $122 \mu \mathrm{Jy} \mathrm{beam}^{-1}$ respectively. The numbered sources have posterior distributions for their flux densities plotted in Fig. 10. See Sect. 4.4 for more details on the plots.

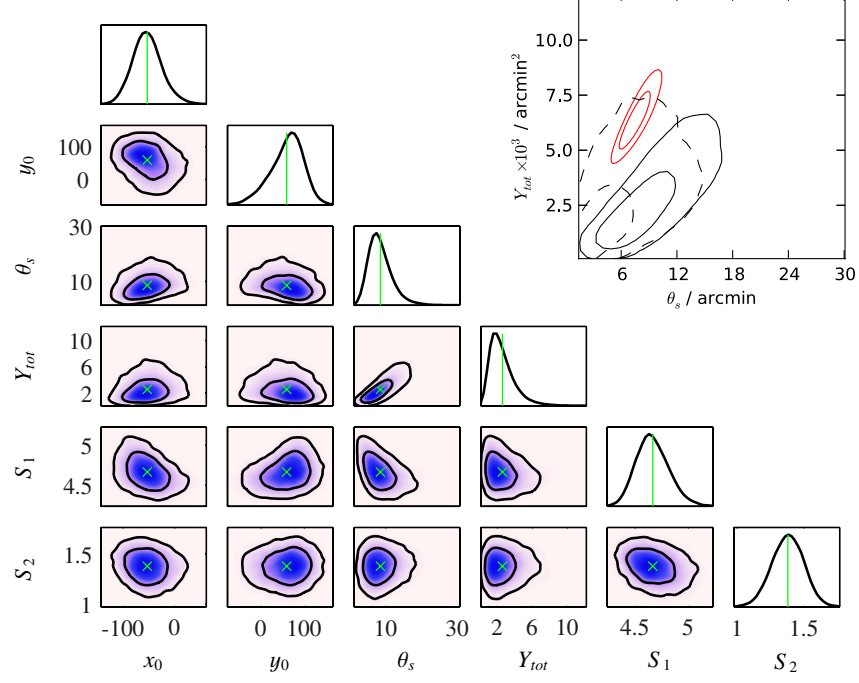

Fig. 10. AMI posterior distributions for ZW8503 and the $Y_{\text {tot }}-\theta_{\mathrm{s}}$ posterior overlaid with that obtained by Planck (upper right hand corner). See Sect. 4.4 for more details on the plots.

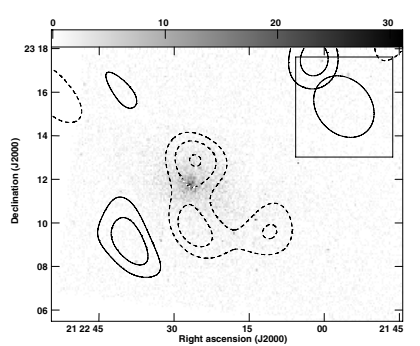

Fig. 11. A Chandra X-ray map of ZW8503 5 with AMI-SA contours at $\pm(2,3,4) \times 100 \mu \mathrm{Jy}$ overlaid to show the substructure. The grey-scale is in units of counts per pixel and is truncated at the peak value in the centre of the cluster. The AMI synthesised beam is shown in the top right-hand corner. Note that the axis scale is different to Fig. 9.

\subsubsection{Non-detections}

PSZ1 G074.75-24.59

PSZ1 G074.75-24.59 is associated in the Planck catalogue with $\mathrm{ZwCl} 2143.5+2014$. Despite having an $\mathrm{S} / \mathrm{N}$ of 6.1 and being detected by all three of the Planck detection algorithms, it is not detected by AMI, with an evidence difference of $\Delta \ln (\mathcal{Z})=-2.6$. 


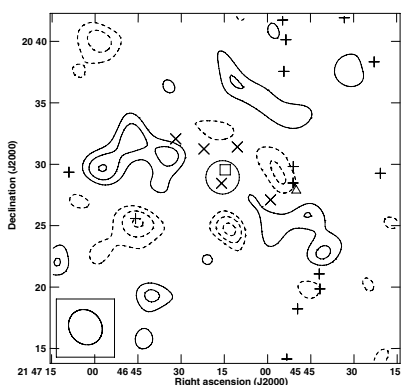

(a)

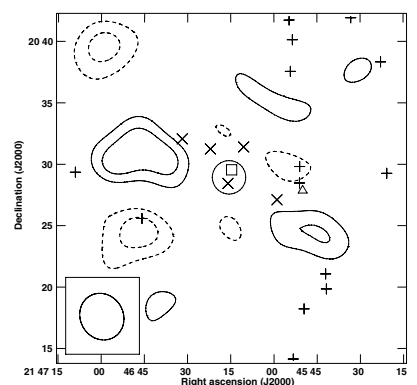

(b)

Fig. 12. SA source-subtracted map of PSZ1 G074.75-24.59 with a) natural weighting and b) a $u v$-taper. The rms noise levels are 105 and $166 \mu \mathrm{Jy}_{\text {beam }^{-1}}$ respectively. The position of $\mathrm{ZwCl} 2143.5+2014$ is shown as a triangle (Zwicky \& Kowal 1968). See Sect. 4.4 for more details on the plots.

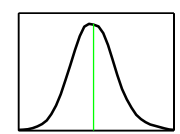

s
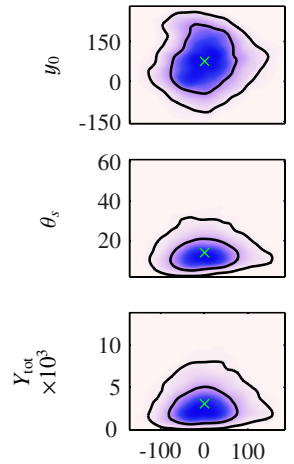

$x_{0}$
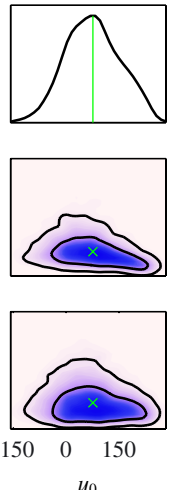
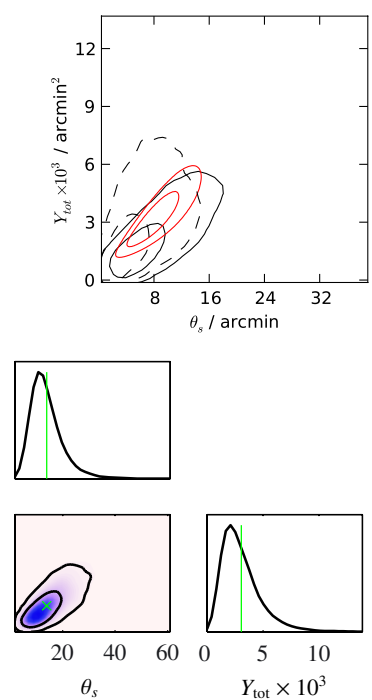

Fig. 13. AMI posterior distributions for PSZ1 G074.75-24.59 and the $Y_{\text {tot }}-\theta_{\mathrm{s}}$ posterior overlaid with that obtained by Planck (upper right hand corner). See Sect. 4.4 for more details on the plots.

Although there is some negative flux visible on the map, it is ruled out by the Planck positional prior (Fig. 12).

A simulated cluster using the PWS maximum a-posteriori values for $\theta_{\mathrm{s}}$ and $Y_{\mathrm{tot}}$, observed using the same visibilities and noise levels as those in the real AMI observation, shows that this cluster should be detected at a $\mathrm{S} / \mathrm{N}$ of $\approx 8$ in the naturallyweighted map, and $\approx 9$ in the $u v$-tapered map. However, the posterior distributions (Fig. 13) show that the $\theta_{\mathrm{s}} / Y_{\text {tot }}$ parameter space preferred by Planck cannot be ruled out by the AMI observations, so the cluster could be more extended than the Planck MAP estimate shows (although the redshift is given as 0.250 so this seems unlikely) and/or be significantly offset from its given position.

\subsubsection{Clear non-detections}

PSZ1 G137.56+53.88 is a clear non-detection with evidence ratio $\Delta \ln (\mathcal{Z})=-4.1$. There is no negative flux near the phase centre and no nearby point sources or positive extended emission to cause the non-detection of the cluster (Fig. 14). Simulations show the cluster should have a significance of $\approx 17$ in both the naturally-weighted and $u v$-tapered maps. The

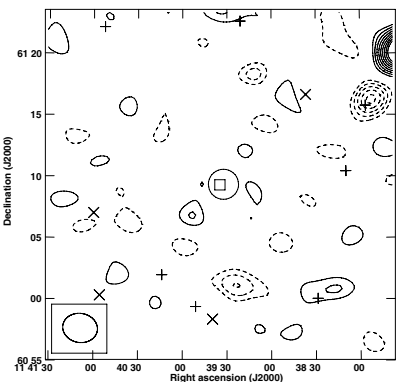

(a)

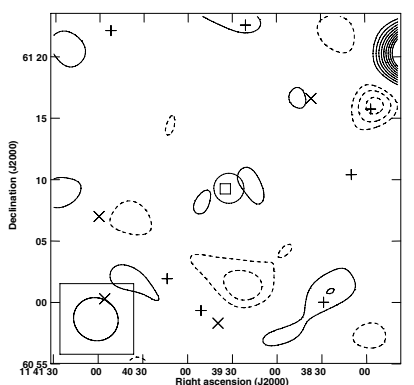

(b)
Fig. 14. SA source-subtracted map of PSZ1 G137.56+53.88 with a) natural weighting and b) a $u v$-taper. The rms noise levels are 109 and $150 \mu \mathrm{Jy}_{\text {beam }}{ }^{-1}$ respectively. See Sect. 4.4 for more details on the plots.
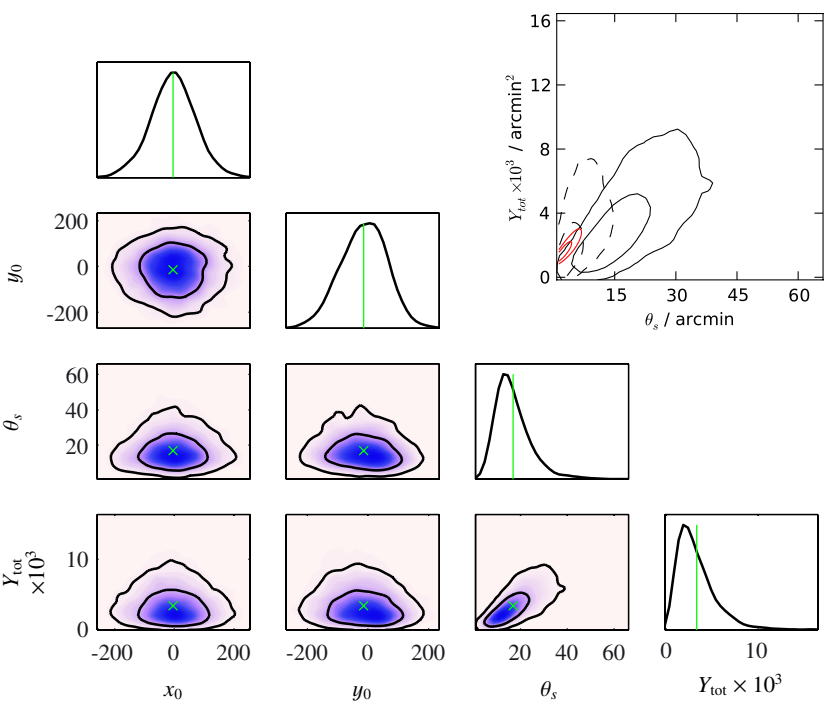

Fig. 15. AMI posterior distributions for PSZ1 G137.56+53.88 and the $Y_{\text {tot }}-\theta_{\mathrm{s}}$ posterior overlaid with that obtained by Planck (upper right hand corner). See Sect. 4.4 for more details on the plots.

posterior distribution (Fig. 15) shows that very large values of $\theta_{\mathrm{s}}$ are required to provide any kind of consistency with the data, so that nearly all of the cluster flux would be resolved out, in disagreement with the small value for $\theta_{\mathrm{s}}$ indicated by PwS. Noting also that although the cluster has an $\mathrm{S} / \mathrm{N}$ of 5.7 , it was detected by PwS only and not the other algorithms, we consider it likely to be a spurious detection.

\subsubsection{Validation}

\section{Detection of new clusters}

Of our SZ sample, 82 clusters are previously known (the validation flag in the Planck catalogue is 20). 16 of the new clusters are already confirmed by other followup (validation $=10$ ); of these, we re-confirm 14.

We detect 14 of the remaining 25 new clusters that have not been previously confirmed by other methods, at the time of publishing of the catalogue. All of these are detected by at least two Planck pipelines, and 8 are detected by all three. For these clusters, the Planck catalogue provides a quality assessment flag between 1 and 3 ( 1 being the most reliable); there are 6, 4 and 4 AMI detections in the 1,2 and 3 categories respectively. 

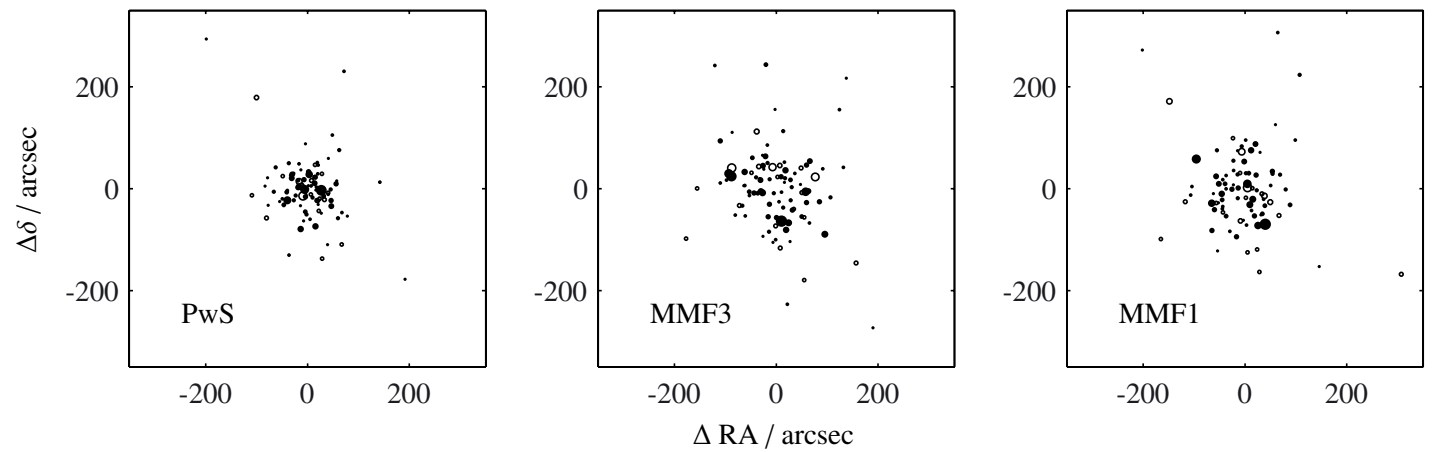

Fig. 16. Positional offset from AMI for the three Planck detection algorithms. The size of the points plotted increases with increasing Planck S/N; clear detections are plotted as filled circles, and moderate detections as empty circles.

\section{Discussion of AMI non-detections}

Across the whole sample, $75 \%$ of the AMI non- and clear nondetections have less than three Planck pipeline detections, compared to $18 \%$ for the AMI clear and moderate detections; of the previously unconfirmed clusters, none of the AMI non- and clear non-detections has a quality flag value of 1 . Although it is difficult to rule out the presence of a cluster entirely using AMI data alone, these correlations indicate that an AMI nondetection is a useful indicator for a possible spurious Planck detection. Figure B.2 shows $\theta_{\mathrm{s}}-Y_{\text {tot }}$ posteriors for all of the nondetections; the Planck parameter space is often ruled out by the AMI posterior.

All of the three clear non-detections have $<3$ Planck pipeline detections. Two of these (PSZ1 G053.50+09.56 and PSZ1 G142.17+37.28) are within 5 arcmin of thermal, compact sources at 545 and/or $857 \mathrm{GHz}$, which are another indicator of a potentially spurious Planck detection caused by contamination by dust emission. The third has been addressed in Sect. 4.4.4; we consider these three likely to be spurious.

The Planck catalogue produced by the intersection of detections by the three algorithms is expected to be $\approx 99 \%$ pure at $S / N \geq 5$ (Planck Collaboration XXIX 2014). Our SZ sample of 123 clusters contains 87 in the intersection catalogue, of which 81 are detected by AMI. This leaves six non-detections. Of these, three (PSZ1 G099.48+55.62, PSZ1 G107.32-31.51, and PSZ1 G084.84+35.04) are at known, low redshift and the posteriors in Fig. B.2 show that the region of $\theta_{\mathrm{s}}-Y_{\text {tot }}$ parameter space preferred by Planck cannot be ruled out by the AMI observations; i.e. these clusters are likely to be too large in angular size (and not bright enough) to be seen by AMI. Of the remaining four, PSZ1 G094.69+26.34 is predicted to have a low $\mathrm{S} / \mathrm{N}$ of $\approx 4$ in the AMI data based on the Planck maximum aposteriori values of $\theta_{\mathrm{s}}$ and $Y_{\mathrm{tot}}$, and could also be resolved out if the true values are toward the upper edge of the constraint. Also, although PSZ1 G050.46+67.54 should be well-detected according to its Planck size estimate of $\theta_{\mathrm{s}} \approx 3 \mathrm{arcmin}$, it is within 220 arcsec of an MCXC cluster with size $\theta_{500}=6.89$ arcmin (Piffaretti et al. 2011), corresponding to $\theta_{\mathrm{s}}=5.85$ arcmin for $c_{500}=1.177$ and may therefore also be resolved out if the Planck size is an under-estimate.

This leaves one cluster only in the intersection catalogue, PSZ1 G074.75-24.59, which simulations based on the Planck maximum a-posteriori parameter estimates predict should be well-detected by AMI; the AMI maps (Fig. 12) show no source environment problems which could explain its non-detection. More follow-up data will be required to definitively determine if this is a spurious detection, as the pressure profile of the cluster gas could deviate significantly from the universal pressure profile and/or the Planck position estimates could be offset significantly from the true position, so that the simulations do not accurately predict the AMI detection significances.

\subsubsection{Positional comparison}

The higher angular resolution of AMI enables a more accurate positional estimate to be produced for the clusters (although in practice this depends on a variety of factors such as signalto-noise over the angular scales observed by both telescopes, and how successful the decoupling of the signal from the foregrounds is). This allows the accuracy of the Planck positions and error estimates to be checked. Figure 16 compares positional offsets between AMI and the three Planck detection algorithms. The offsets for MMF1 and MMF3 are very similar. The PWS offsets are slightly more clustered toward zero, and also show a greater correlation with the $\mathrm{S} / \mathrm{N}$ (i.e. the highest $\mathrm{S} / \mathrm{N}$ points are closer to zero than the low-S/N points).

The MMF1 algorithm does not currently output positional errors, so Fig. 17 shows the distribution of positional offsets normalised by the total error $\left(\sqrt{\sigma_{\text {AMI }}^{2}+\sigma_{\text {Planck }}^{2}}\right)$ for PWS and MMF3 only. A Rayleigh distribution, $\left(x / \sigma^{2}\right) \exp \left(-x^{2} / 2 \sigma^{2}\right)$ with $\sigma=1$, is plotted for comparison - this is the expected distribution assuming the errors in RA and $\delta$ are uncorrelated and normally distributed. The PWS distribution is a reasonable match, showing that the error estimates are a good representation of the true uncertainty in the positions. In contrast, the MMF3 errors are generally overestimated in this version of the Planck catalogue.

We estimate a rescaling factor of 0.28 for the MMF3 errors, by minimising the Kolmogorov-Smirnov test statistic between the distribution and the Rayleigh distribution. Figure 18 shows the rescaled histogram, which agrees much more closely with the Rayleigh distribution. In contrast, the same procedure gives a rescaling factor of 0.51 for the PwS errors. Figure 18 also shows a comparison between the absolute offsets between AMI and PwS and AMI and MMF3; confirming what is seen in Fig. 16, the PwS offsets are generally smaller, especially at high $\mathrm{S} / \mathrm{N}$.

The MMF3 rescaling factor is in agreement with that estimated via internal Planck quality assessment, and later versions of the catalogue have been corrected for this ${ }^{6}$.

\footnotetext{
6 See http://wiki.cosmos.esa.int/planckpla/index.php/ Catalogues\#The_SZ_catalogues under Caveats.
} 

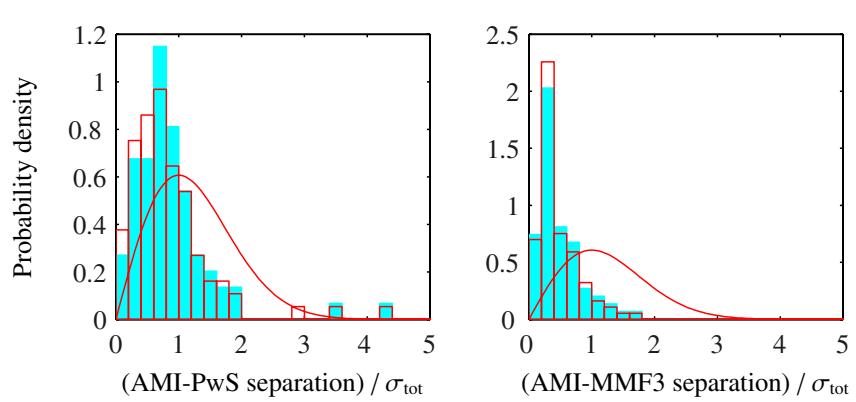

Fig. 17. Positional offset from AMI, normalised by total error $\sigma_{\text {tot }}=$ $\sqrt{\sigma_{\mathrm{AMI}}^{2}+\sigma_{\text {Planck }}^{2}}$, for PwS and MMF3. The solid histogram shows the clear detections only, and the red outline shows clear and moderate detections together. A Rayleigh distribution is plotted in red for comparison.

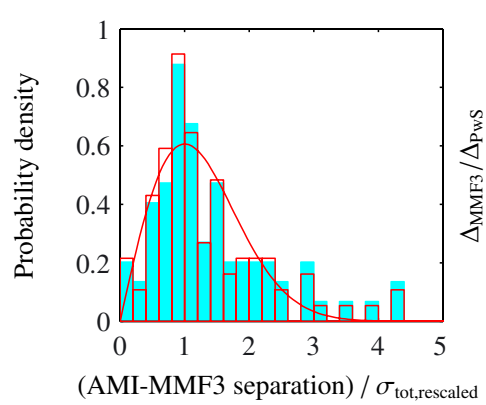

(a)

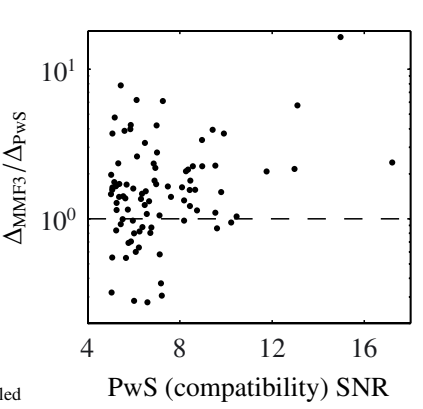

(b)
Fig. 18. a) shows the MMF3 positional offset from AMI, normalised by rescaled total error $\sigma_{\text {tot }}=\sqrt{\sigma_{\mathrm{AMI}}^{2}+\left(0.28 \times \sigma_{\mathrm{MMF} 3}\right)^{2}}$. The solid histogram shows the clear detections only, and the red outline shows clear and moderate detections together. A Rayleigh distribution is plotted in red for comparison. b) shows the ratio between the absolute offsets $(\Delta)$ between AMI and MMF3 and AMI and PwS as a function of $\mathrm{S} / \mathrm{N}$; as shown in Fig. 16, PwS does better at high $\mathrm{S} / \mathrm{N}$.

\subsection{7. $Y_{\text {tot }}-\theta_{\mathrm{s}}$ comparison}

A major conclusion of AP2013 was that the clusters were found overall to be smaller in angular size and fainter (lower $Y_{\text {tot }}$ ) by AMI than by Planck. The comparison for the larger sample shows a similar trend.

To properly compare the quantities, it is necessary to look at the full, two-dimensional posteriors for $Y_{\text {tot }}$ and $\theta_{\mathrm{s}}$ since the quantities are correlated. Figure B.1 shows the two-dimensional posteriors for $\theta_{\mathrm{s}}$ and $Y_{\mathrm{tot}}$ as measured by both AMI and Planck, and the joint constraints where appropriate, in descending Planck S/N order. It is clear that, especially at the high-S/N end, there are many cases where the constraints are inconsistent and in these cases the Planck posteriors usually prefer higher values of $\theta_{\mathrm{s}}$ and $Y_{\mathrm{tot}}$.

Figure 19 shows the comparison between the AMI and PwS mean values for the entire sample of clear and moderate detections. Aside from some outliers, the $\theta_{\mathrm{s}}$ values do not seem to be biased, but only correlate weakly, with a Pearson correlation coefficient of 0.25 (0.18) for all common AMI and PwS detections (clear AMI detections only). However, the $Y_{\text {tot }}$ values for the high-S/N clusters as measured by AMI are still lower overall than the Planck values; for lower S/N clusters, the bias may be obscured by the noise. Following Planck Collaboration XX (2014) for the definition of high-S/N, we make a cut at Planck $\mathrm{S} / \mathrm{N}$ of 7 and fit a linear model to the Planck and AMI results for
$Y_{\text {tot }}$, using the ScIPY orthogonal distance regression function ${ }^{7}$ to take into account errors in both the $x$ and $y$ direction. The best fit slope for all clusters (clear AMI detections only) above S/N of 7 is $4.2 \pm 1.5(2.45 \pm 0.72)$; note that the slope for all clusters is driven by one very discrepant moderate detection. The slope for clear AMI detections only is consistent with the slope found in AP2013 $(1.05 \pm 0.05)$ at $<2 \sigma$ significance; note however that this relationship was obtained by fixing the cluster size to the $\theta_{500}$ inferred from the X-ray luminosity for improved consistency.

The comparison between AMI values and the values produced by the MMF algorithms is very similar.

This inconsistency could be due to the fact that AMI does not measure $Y_{\text {tot }}$ directly, since it is an interferometer and therefore resolves out the larger scales; as long as the cluster is resolved, the zero-spacing flux, and therefore $Y_{\text {tot }}$, is never measured directly. In this case the discrepancy should be worse for larger angular-size clusters since more of an extrapolation is required to infer the zero-spacing flux. In Fig. 20a, the ratio of the $Y_{\text {tot }}$ values is plotted as a function of $\theta_{\mathrm{s}}$ as measured by AMI and Planck; the discrepancy does appear worse for larger values of $\theta_{\mathrm{s}, \text { Planck }}$, but occurs across all values of $\theta_{\mathrm{s}, \mathrm{AMI}}$. In Fig. $20 \mathrm{~b}$ the correlation between $\theta_{\mathrm{s}}$ and $Y_{\text {tot }}$ is plotted as measured by AMI and Planck, which also shows that the discrepancy occurs over the entire sample.

\section{Potential origins of the discrepancy}

To first eliminate the possibility that the discrepancy is caused by absolute calibration problems, we obtained flux densities for two of our primary calibration sources, 3C 286 and 3C 147, at 30 and $44 \mathrm{GHz}$ from the Planck Compact Source Catalogue (Planck Collaboration XXVIII 2014). These are shown in Fig. 21 with the power-law used to calculate the AMI primary calibration flux densities for comparison. All flux densities are within $3 \sigma$ of the power-law, and there does not appear to be a systematic bias. We therefore discard absolute calibration as a potential cause of the discrepancy.

Several potential origins of the discrepancy were investigated in AP2013, as follows.

1. The possibility that a population of faint sources existed below the LA detection threshold and acted to fill in the decrement was investigated by obtaining very deep LA observations toward the central pointing of the raster for each cluster, obtaining rms noise levels $\lesssim 30 \mu \mathrm{Jy} \mathrm{beam}^{-1}$, and re-extracting the cluster parameters, subtracting any extra sources detected. In one case this shifted the $Y_{\text {tot }}$ estimate upward by $\approx 1 \sigma$, but the parameters for the remaining 10 cases were not significantly changed. This is clearly not the source of the discrepancy.

2. To eliminate any effects from differing centroid positions, the AMI and Planck data were both analysed with the position of the cluster fixed to the best-fit position obtained from an initial AMI analysis where the central position was allowed to vary. Fixing the position also had a negligible effect on the derived $\theta_{\mathrm{s}}$ and $Y_{\text {tot }}$ posterior distributions.

3. For five clusters with measured $X$-ray profiles, the cluster parameters were re-extracted using the appropriate X-raydetermined $\gamma$ and $\alpha$ parameters rather than the universal parameters. This did not significantly improve the agreement. Note that the parameter affecting the cluster outskirts, $\beta$, was

http://docs.scipy.org/doc/scipy/reference/odr.html 

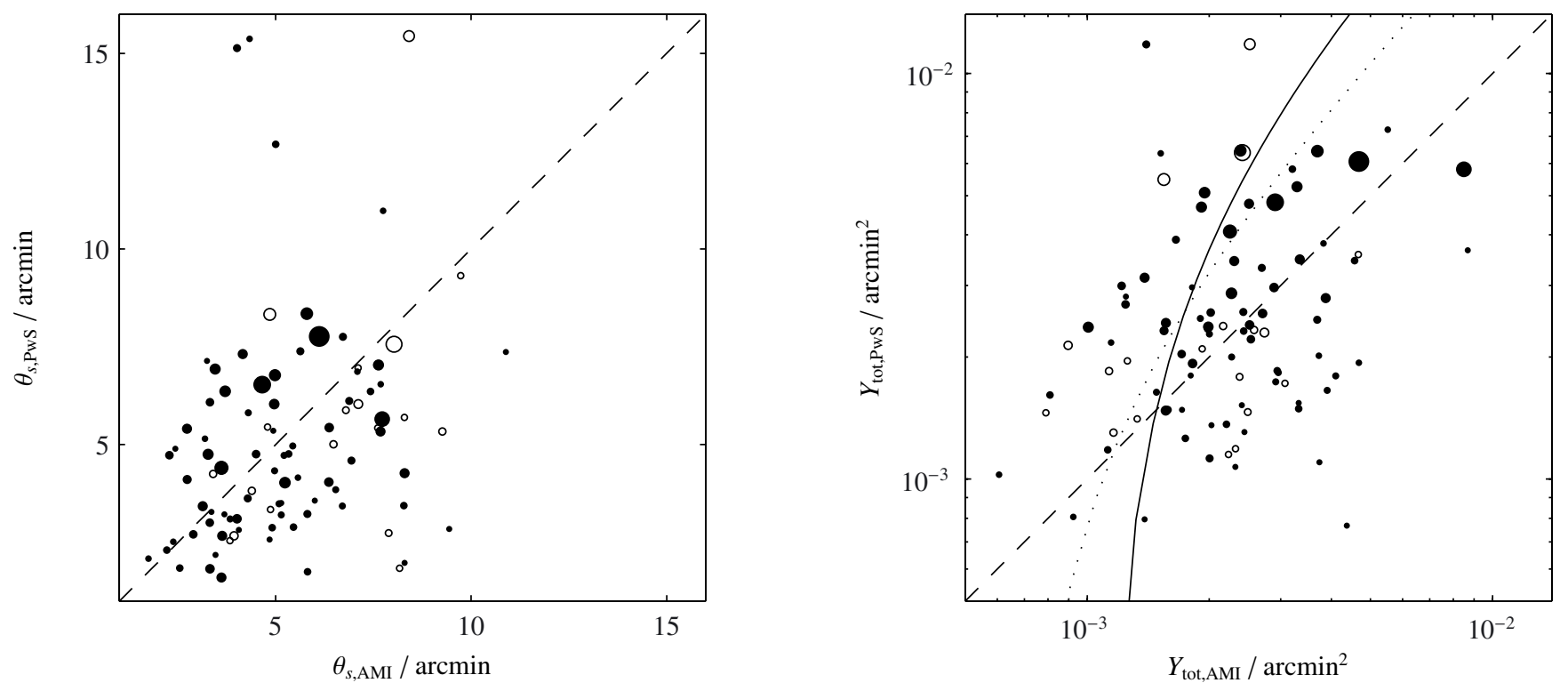

Fig. 19. Comparison between PwS and AMI mean $Y_{\text {tot }}$ and $\theta_{\mathrm{s}}$ values. The size of the points plotted increases with increasing Planck S/N; clear detections are plotted as filled circles, and moderate detections as empty circles. Error bars are omitted for clarity and since the errors in $Y_{\text {tot }}$ and $\theta_{\mathrm{s}}$ are correlated. The one-to-one relationship is plotted as a black dashed line. The fitted linear relationship for all clusters (clear AMI detections only) with $\mathrm{S} / \mathrm{N}$ greater than 7 is plotted as a black solid (black dotted) line.

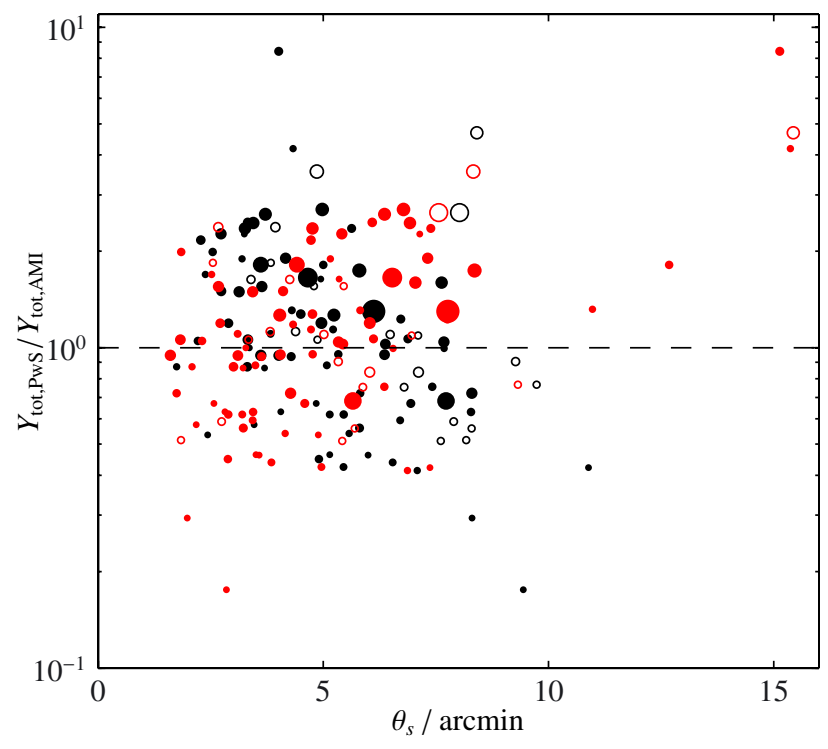

(a)

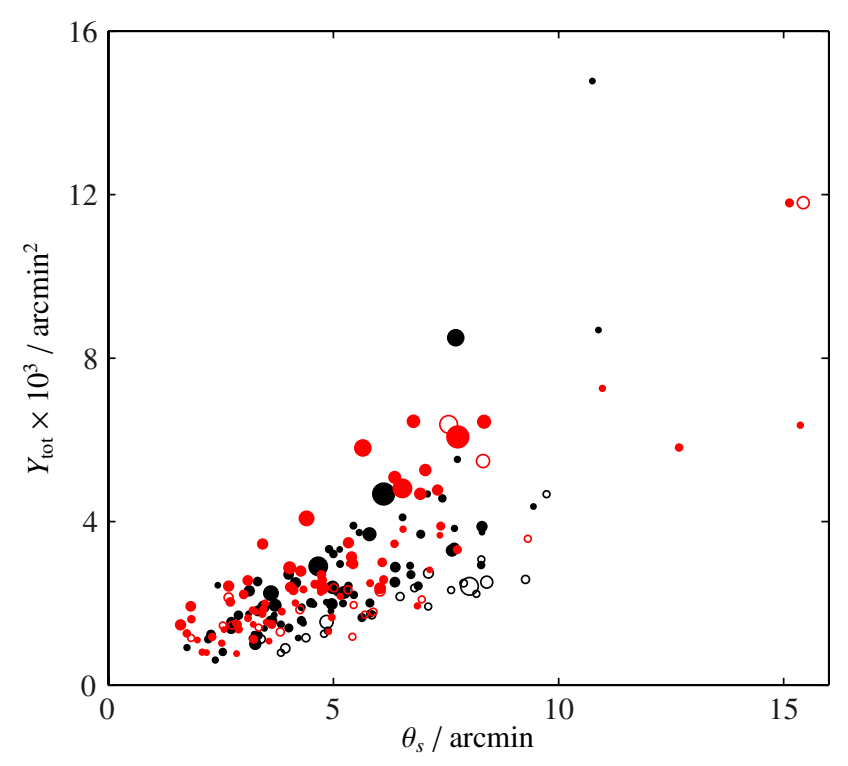

(b)

Fig. 20. a) Shows a comparison between PwS and AMI MAP $Y_{\text {tot }}$ values as a function of AMI (PwS) $\theta_{\mathrm{s}}$ values in black (red). The one-to-one relationship is plotted as a black dashed line. b) Shows $Y_{\text {tot }}$ as a function of $\theta_{\mathrm{s}}$ as measured by AMI (black) and PwS (red) for all of the moderate and clear detections. In both plots, the size of the points plotted increases with increasing Planck S/N, clear detections are plotted as filled circles, and moderate detections as empty circles. Error bars are omitted for clarity and since the errors in $Y_{\mathrm{tot}}$ and $\theta_{\mathrm{s}}$ are correlated.

not varied since the X-ray data do not extend to this region. See AP2013 for more details.

When a point source very near the cluster centre is fitted simultaneously with the cluster model, there is often a correlation between the point source flux and the $Y_{\text {tot }}$ value, i.e. the data can constrain the sum of the point source flux and the cluster flux well, but not separate the two components. If this effect led to biases in the fitted $Y_{\text {tot }}$ values, it would worsen for smaller angular-size clusters since it becomes more difficult to distinguish between the profiles in $u v$-space of a marginally-resolved cluster and an unresolved point source. To test whether this could cause the discrepancy, we replotted Fig. 20 using only clusters with no fitted sources within 3 arcmin of the cluster position. This is shown in Fig. 22; although the number of clusters in the plot is much smaller, the discrepancy is clearly not resolved. In addition, we conducted tests on simulations of clusters with point sources of varying flux densities and at varying distances from the cluster centres, and found that we were able to recover $Y_{\text {tot }}$ values correctly.

Another potential problem is the mismatch between the spherical model and the real data; the higher resolution AMI data will be much more sensitive to this issue than the Planck data 


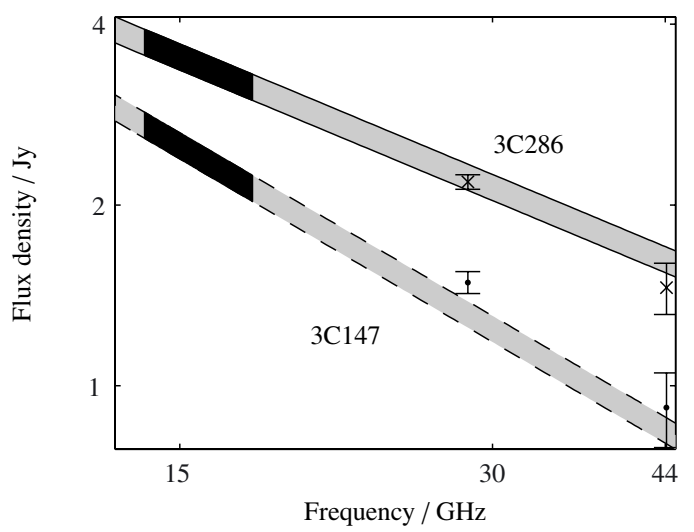

Fig. 21. Power-law relationships used to calculate primary calibration flux densities for AMI for two calibrators, 3C 286 and 3C 147, are shown with $\pm 5 \%$ uncertainty limits as the grey filled bands. The AMI frequency band is shown in black. Flux densities for both sources at 30 and $44 \mathrm{GHz}$ taken from the Planck Compact Source Catalogue (Planck Collaboration XXVIII 2014) are shown as points with errorbars.

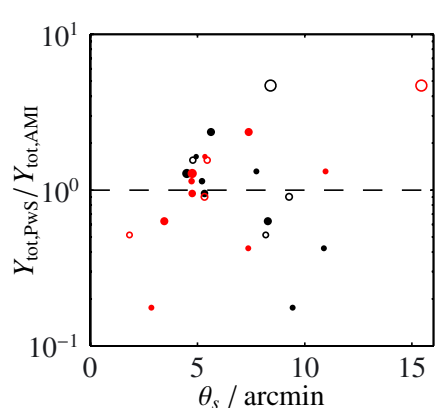

(a)

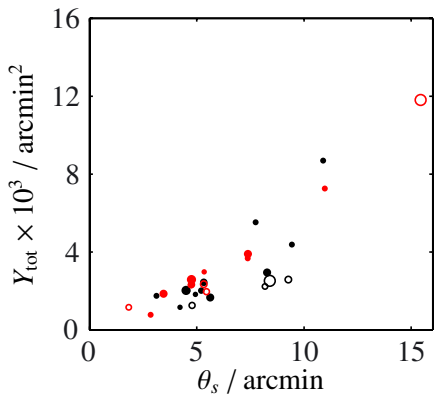

(b)
Fig. 22. Comparison between PWS and AMI $\theta_{\mathrm{s}}$ and $Y_{\text {tot }}$ MAP values, for clusters selected to have no radio point sources within 3 arcmin of the cluster position. In both plots, the black (red) points show the AMI (PwS) values, larger points have higher Planck S/N values and filled (empty) circles represent AMI clear (moderate) detections.

(in some cases, also dependent on other factors as discussed in Sect. 4.4.6). Some of the clusters have clearly non-spherical shapes in the AMI maps, but modelling with an ellipsoidal GNFW profile does not change the constraints on $Y_{\text {tot }}$ and $\theta_{\mathrm{s}}$ significantly.

\section{Profile investigation}

The outstanding issue to be considered is the use of the universal profile shape for all clusters. AMI-SA data are not of high enough resolution to measure $\gamma$; the range of scales measured by the SA corresponds to $0.3 \lesssim \theta / \theta_{\mathrm{s}} \lesssim 9$ for clusters with angular sizes $\theta_{\mathrm{s}}$ in the range 2 to $10 \mathrm{arcmin}$. For the smallest (largest) clusters in the sample, $\alpha(\beta)$ will be the parameter most affecting AMI data; for most clusters, both will be important.

\subsection{Analysis of simulations}

As a first step to understanding how variation in the shape parameters affects constraints derived from AMI data, we generated a set of simulations with realistic thermal, CMB and source confusion noise levels. We chose three representative values of $\theta_{\mathrm{s}}$ based on the follow-up sample, and assigned realistic $Y_{\text {tot }}$ values to each based on clusters in the sample with a similar angular size and that were well-detected by AMI, giving $\left(\theta_{\mathrm{s}}, Y_{\text {tot }}\right)=(1.8,0.0009),(4.5,0.001)$ and $(7.4,0.007)$. For each $\left(\theta_{\mathrm{s}}, Y_{\mathrm{tot}}\right)$, we generated simulations with $\alpha$ and $\gamma$ set to the 31 individual fitted values from the REXCESS sample (Böhringer et al. 2007; Arnaud et al. 2010), and with $\beta$ drawn from a uniform distribution between 4.5 and 6.5. Figure 23a shows the result of analysing these simulations with the standard AMI analysis pipeline, assuming the universal profile parameters, whereas Fig. $23 \mathrm{~b}$ shows the results when the simulation is both generated and analysed with the universal profile. In the former case, for the two smaller clusters, the true value is within the $68 \%$ confidence limit 29 times out of 31 , but it is clear that the size and degeneracy direction of the contours varies wildly for different sets of $(\gamma, \alpha, \beta)$; on the whole, the mean and MAP values of $\theta_{\mathrm{s}}$ and $Y_{\text {tot }}$ are biased upward slightly. For the largest cluster, the true value is within the $68 \%$ confidence limit only 2 times out of 31, and within the $95 \%$ confidence limit only 14 out of 31 times. Again, the size and degeneracy directions of the contours vary wildly; note that the very tight contours which are significantly discrepant from the rest correspond to the profile in the REXCESS sample that is most discrepant from the universal profile, with shape parameters $\gamma=0.065, \alpha=0.33$. On the whole, the mean and MAP values of $\theta_{\mathrm{s}}$ and $Y_{\text {tot }}$ are biased downward significantly for this cluster.

To assess the potential for constraining $\alpha$ and $\beta$ using AMI data, we next analysed the simulations, allowing the shape parameters to vary one at a time and using wide, uniform priors on all parameters. We found that, due to the lack of information on $Y_{\text {tot }}$ in the data, there are very strong degeneracies between $\theta_{\mathrm{s}}$ and $\alpha$ and $\beta$, even when data with very small amounts of noise are analysed. For example, Fig. 24 shows that a profile generated with the universal value of $\beta$ and a small angular size can be mimicked almost identically across a given range of angular scales using a much larger $\beta$ and $\theta_{\mathrm{s}}$ value.

In practice, these strong degeneracies were found to lead to spurious constraints in $\alpha$ and $\beta$ in the one-dimensional marginalised posterior distribution. This is simply due to the shape of the three-dimensional posterior; more $Y_{\text {tot }}-\theta_{\mathrm{s}}$ space becomes available for lower values of $\alpha$ and $\beta$. Applying the twodimensional prior on $Y_{\text {tot }}$ and $\theta_{\mathrm{s}}$ to ensure that physically motivated parts of the $Y_{\mathrm{tot}}-\theta_{\mathrm{s}}$ space are selected reduces, but does not eliminate, the problem. This is illustrated in Figs. 25 and 26 where the two- and one-dimensional posterior distributions are shown for $\theta_{\mathrm{s}}, Y_{\text {tot }}$ and $\alpha$, with the standard two-dimensional prior on $\theta_{\mathrm{s}}$ and $Y_{\text {tot }}$ and a uniform prior between 0.1 and 3.0 on $\alpha$ (with $\beta$ fixed to the correct, input value of 5.4905). When there is little information on $\alpha$ in the data (particularly for the smallest cluster), the shape of the two-dimensional posteriors produces an apparent (and incorrect) constraint on $\alpha$ in the one-dimensional posteriors. Similar effects occur in the constraints on $\beta$, shown in Figs. 27 and 28 (in which $\alpha$ is fixed to the correct, input value of 1.0510).

To attempt to control these biases, we reanalysed the simulations using a Gaussian prior based on the REXCESS sample on $\alpha$, namely $\mathcal{N}(1.0510,0.47)$ truncated at 0.3 , and a tighter uniform prior on $\beta, \mathcal{U}[4.5,6.5]$. Figure 29 shows the resulting posterior distributions, varying both $\alpha$ and $\beta$ (but with $\gamma$ fixed to the universal value). For the two smaller angular-size clusters, this results in correct recovery of $\theta_{\mathrm{s}}$ and $Y_{\text {tot }}$, and reduces the biassing considerably in $\alpha$ and $\beta$. For the largest angularsize cluster, the input values of $\theta_{\mathrm{s}}$ and $Y_{\text {tot }}$ are not recovered 

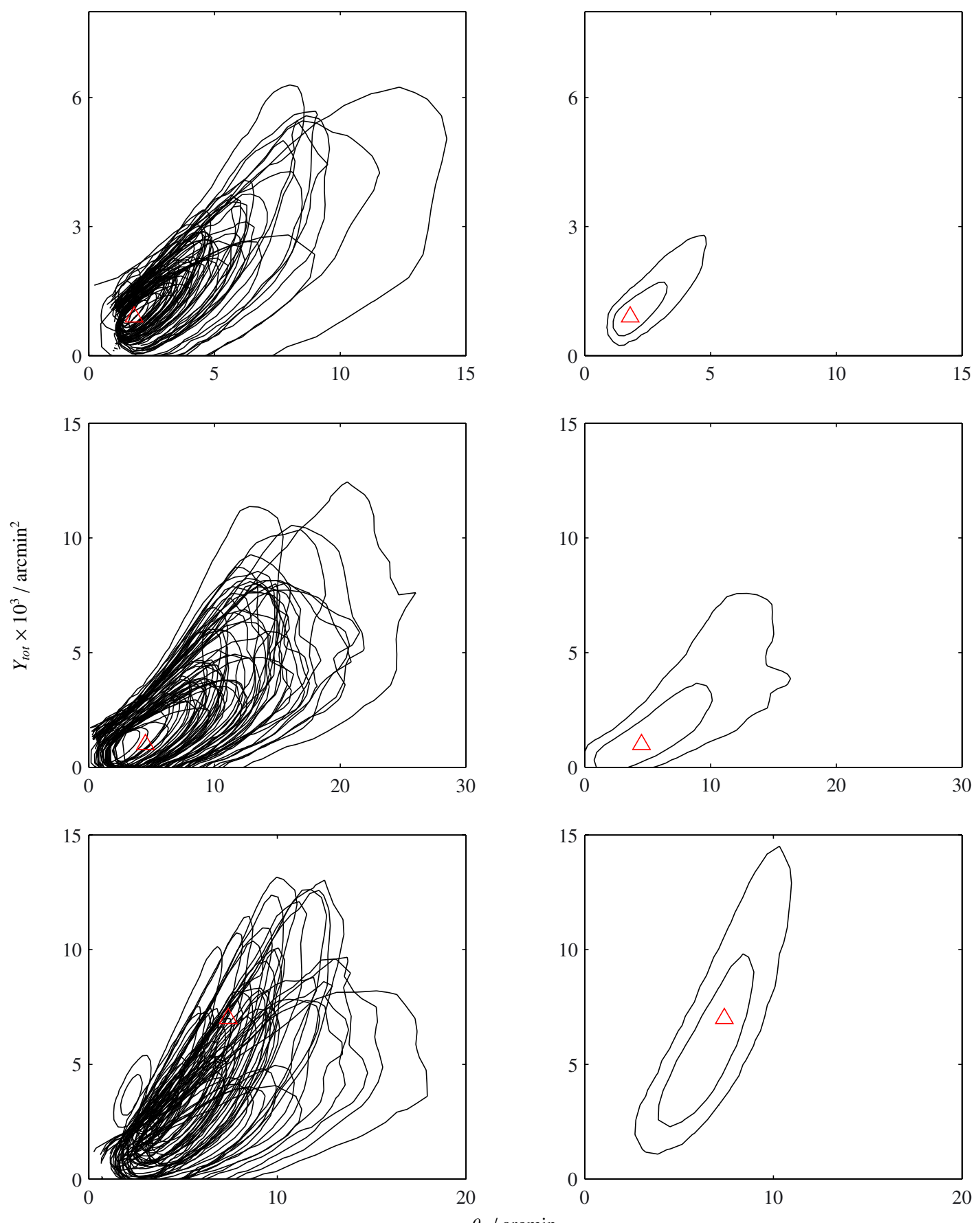

(a)

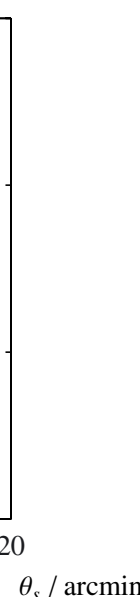

$\theta_{s} / \operatorname{arcmin}$

(b)

Fig. 23. Posterior distribution for $Y_{\mathrm{tot}}$ and $\theta_{\mathrm{s}}$ for simulated clusters with realistic CMB and noise levels (see text for details), and a) differing GNFW shape parameter values $(\gamma, \alpha, \beta)$ based on the REXCESS sample (Böhringer et al. 2007; Arnaud et al. 2010); and b) simulated with the universal values. In all cases the model used for recovering the parameters has the shape parameter values fixed to the universal values, and the joint twodimensional prior on $Y_{\mathrm{tot}}$ and $\theta_{\mathrm{s}}$ is used. Results for three different angular sizes are shown (from top to bottom, $\theta_{\mathrm{s}}=1.8,4.5$ and 7.4); the input parameter values are marked with red triangles. The contours are at the $68 \%$ and $95 \%$ confidence boundaries.

correctly, because there is not enough information available in the angular scales measured by the SA to constrain these parameters simultaneously, so the prior on $Y_{\text {tot }}$ and $\theta_{\mathrm{s}}$ biases the recovered posteriors downwards.

We check for any biases due to $\gamma$ being fixed (incorrectly) to the universal value by plotting the error in the recovered values of $\theta_{\mathrm{s}}$ and $Y_{\text {tot }}$ as a function of the true input $\gamma$ value. There is some correlation between the fractional difference in $\theta_{\mathrm{s}}$ and $\gamma$, especially for the two smaller clusters, but mostly any correlation is beneath the level of the noise (Fig. 30).

We also add point sources of varying flux densities and at varying distances from the phase centre to test for any issues in decorrelating point source flux from cluster flux when varying the shape parameters; the parameter estimation is unaffected. 


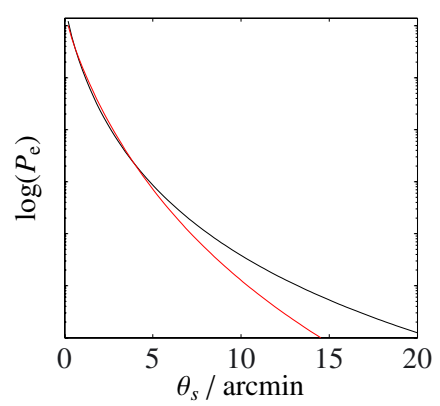

(a)

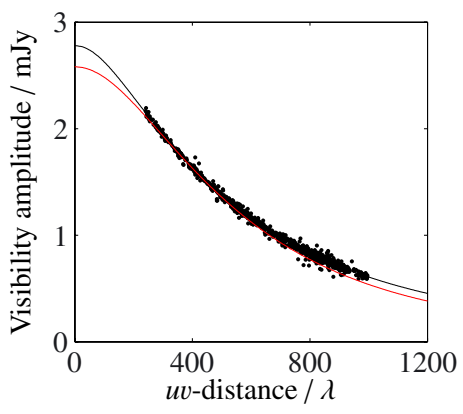

(b)
Fig. 24. A profile generated with $\beta=5.4905, \theta_{\mathrm{s}}=1.8$ (the universal profile, black lines) can be mimicked for $\beta=8.9$ using $\theta_{\mathrm{s}}=4.1$ and adjusting $Y_{\text {tot }}$ downward (red lines). The two profiles are almost identical over the AMI-SA range of baselines, while Planck would measure the zero-spacing flux which differs by $\approx 7 \%$ between the two models. a) Shows the pressure profiles in radial coordinates (note that the $y$-axis scale is $\log$ ); and b) shows the profiles in $u v$-space for channel 5, with the simulated AMI data shown as dots. Note that this simulation has been generated with an unrealistically small amount of thermal noise.
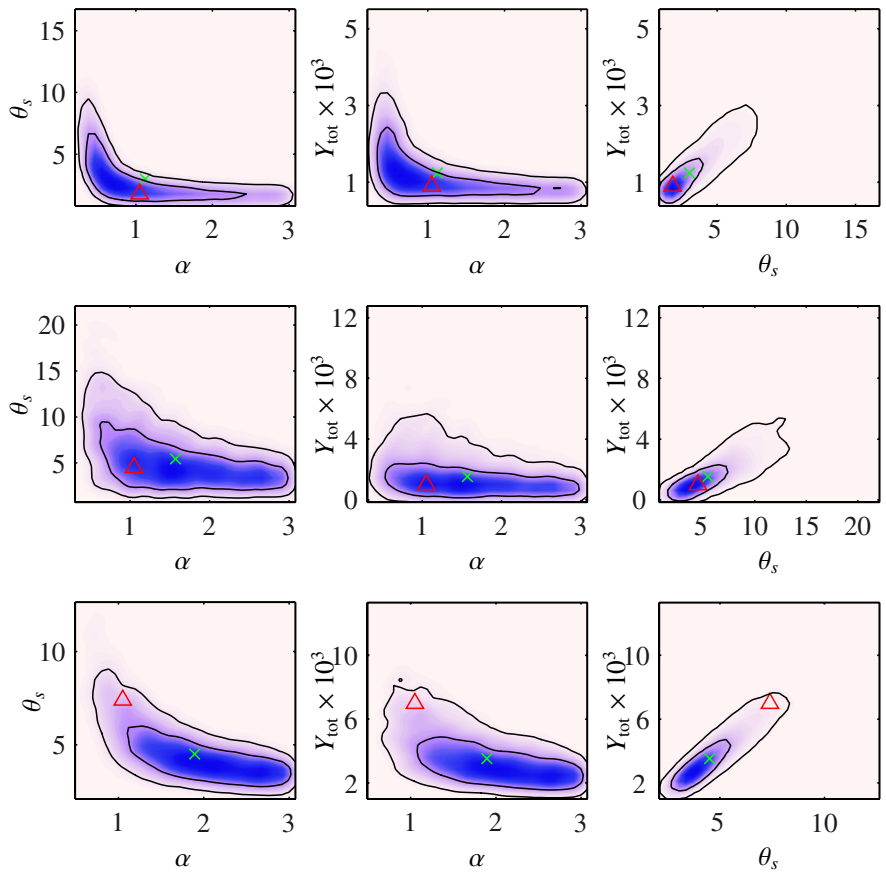

Fig. 25. Posterior distributions for $Y_{\text {tot }}, \theta_{\mathrm{s}}$ and $\alpha$ for simulated low-noise data, for clusters with $\theta_{\mathrm{s}}=1.8$ (top), 4.5 (centre) and 7.4 (bottom) arcmin and universal $(\gamma, \alpha, \beta)$, with the two-dimensional prior on $Y_{\text {tot }}$ and $\theta_{\mathrm{s}}$ and a uniform prior on $\alpha$ between 0.1 and 3.0 ( $\beta$ fixed to the correct, input value). The input values are indicated by red triangles, and the posterior means with green crosses. $\theta_{\mathrm{s}}$ is in arcmin and $Y_{\text {tot }}$ is in $\operatorname{arcmin}^{2}$.

\subsubsection{Adding Planck information}

Although the immediate issue is to check whether we can achieve consistency between AMI and Planck results, it is also interesting to consider whether we can take advantage of the complementary nature of the two instruments to derive better constraints on the behaviour of the pressure profile over a range of radii. To this end, for each of our three simulated cluster sizes we derived a Planck-like prior on $Y_{\text {tot }}$ by marginalising over the $\theta_{\mathrm{s}}$ dimension of the two-dimensional constraint produced by Planck for a cluster with similar angular size, and
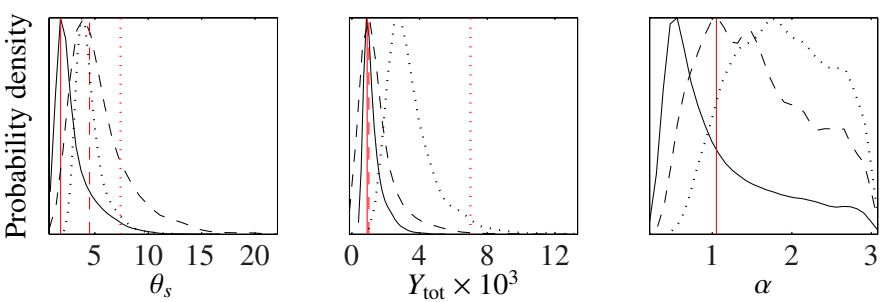

Fig. 26. One-dimensional marginal constraints on $Y_{\mathrm{tot}}, \theta_{\mathrm{s}}$ and $\alpha$ for simulated low-noise data, for clusters with $\theta_{\mathrm{s}}=1.8$ (solid lines), 4.5 (dashed lines) and 7.4 (dotted lines) arcmin and universal $(\gamma, \alpha, \beta)$, with the two-dimensional prior on $Y_{\text {tot }}$ and $\theta_{\mathrm{s}}$ and a uniform prior on $\alpha$ between 0.1 and 3.0 ( $\beta$ fixed to the correct, input value). Input values are shown as red lines. $\theta_{\mathrm{s}}$ is in $\operatorname{arcmin}$ and $Y_{\mathrm{tot}}$ is in $\operatorname{arcmin}^{2}$.
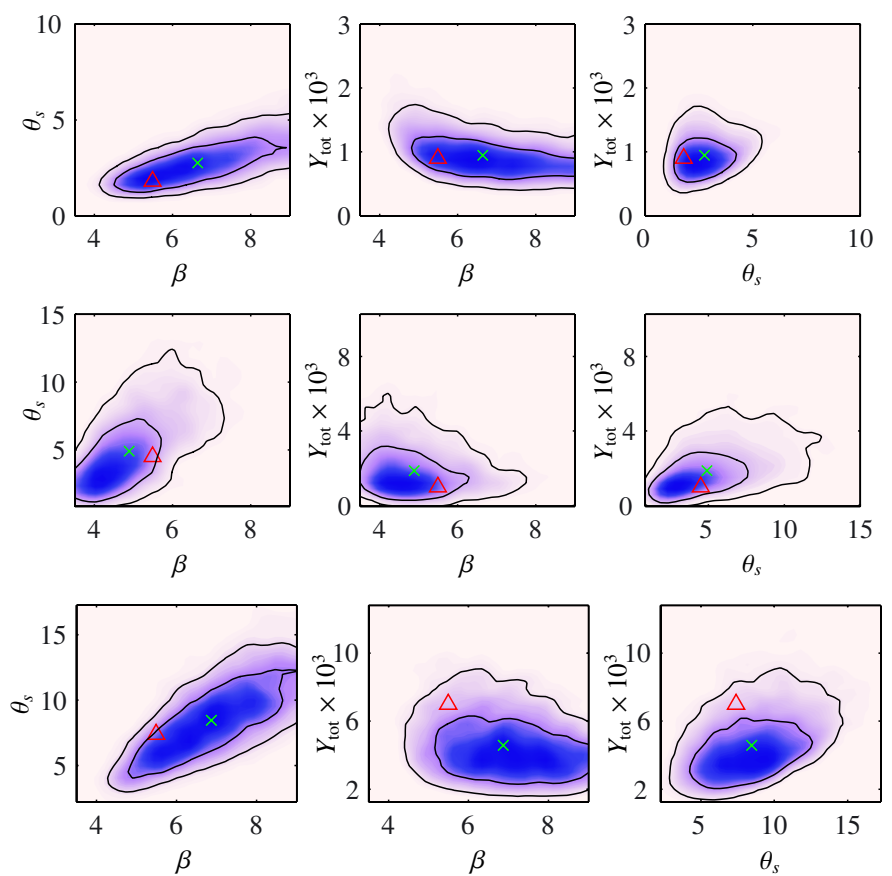

Fig. 27. Posterior distributions for $Y_{\mathrm{tot}}, \theta_{\mathrm{s}}$ and $\beta$ for simulated lownoise data, for clusters with $\theta_{\mathrm{s}}=1.8$ (top), 4.5 (centre) and 7.4 (bottom) arcmin and universal $(\gamma, \alpha, \beta)$, with the two-dimensional prior on $Y_{\mathrm{tot}}$ and $\theta_{\mathrm{s}}$ and a uniform prior on $\beta$ between 3.5 and 9.0 ( $\alpha$ fixed to the correct, input value). The input values are indicated by red triangles, and the posterior means with green crosses. $\theta_{\mathrm{s}}$ is in arcmin and $Y_{\text {tot }}$ is in $\operatorname{arcmin}^{2}$.

approximating as a Gaussian. We use this marginalised constraint as a prior rather than the full two-dimensional constraint since Planck $Y_{\text {tot }}$ estimation is more robust to changes in the profile shape parameters (Harrison et al. 2015). We then use our standard two-dimensional prior on $\theta_{\mathrm{s}}$ conditioned on values drawn from the Planck-like $Y_{\text {tot }}$ prior; priors on $\alpha$ and $\beta$ are as in the previous section. Figure 31 shows the resulting posterior distributions. For all three clusters, the constraints on $\theta_{\mathrm{s}}$ and $Y_{\text {tot }}$ are much tighter, and for the large angular-size cluster, the true values of $\theta_{\mathrm{s}}$ and $Y_{\text {tot }}$ are now recovered correctly. However, the constraints on the shape parameters are not very different.

This is a fairly crude way of including Planck information in the analysis and does not make the best use of the information available in the Planck data on the cluster shape. A full joint analysis of AMI and Planck data would fill in the gap in $u v$ coverage between the zero-spacing flux and the shortest AMISA baselines, and there would be some overlap with the shortest baselines since the resolution of Planck is $\approx 5$ arcmin; this should 

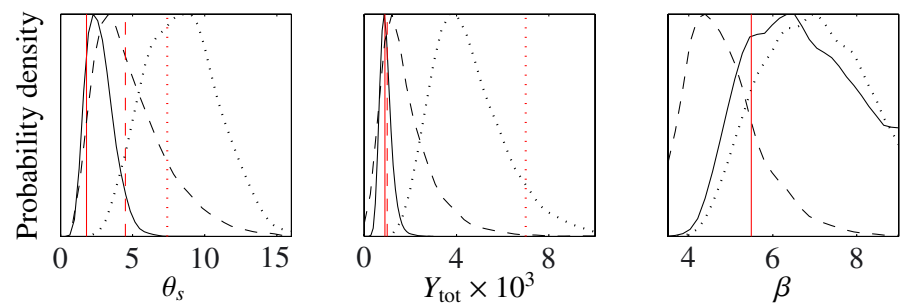

Fig. 28. One-dimensional marginal constraints on $Y_{\mathrm{tot}}, \theta_{\mathrm{s}}$ and $\beta$ for simulated low-noise data, for clusters with $\theta_{\mathrm{s}}=1.8$ (solid lines), 4.5 (dashed lines) and 7.4 (dotted lines) arcmin and universal $(\gamma, \alpha, \beta)$, with the twodimensional prior on $Y_{\mathrm{tot}}$ and $\theta_{\mathrm{s}}$ and a uniform prior on $\beta$ between 3.5 and 9.0 ( $\alpha$ fixed to the correct, input value). Input values are shown as red lines. $\theta_{\mathrm{s}}$ is in arcmin and $Y_{\mathrm{tot}}$ is in $\operatorname{arcmin}^{2}$.

produce better constraints on the profile shape parameters. This will be addressed in a future paper.

\subsection{Summary of simulation results}

We have shown with the simulated bank of clusters based on the REXCESS sample, that when a cluster has an angular size $\theta_{\mathrm{s}} \gtrsim 5$ arcmin, the true input values of $\theta_{\mathrm{s}}$ and $Y_{\text {tot }}$ can only be recovered correctly using AMI data when the model for the pressure profile used for parameter extraction is a good match to the actual pressure profile of the cluster. This is not surprising since, as we have mentioned, an interferometer does not measure zerospacing flux directly and so the $Y_{\text {tot }}$ value measured by AMI is actually an extrapolation based on the assumed profile. This is also consistent with what we observe in the real sample; clusters with high Planck $\mathrm{S} / \mathrm{N}$ (and therefore large $\theta_{\mathrm{s}}$ ) are consistently measured to be smaller and fainter by AMI.

When attempting to vary the GNFW shape parameters, we must be careful to avoid over-interpretation of apparent constraints on parameters which are actually just caused by the shape of the two-dimensional degeneracies. Reducing the range of $\beta$ and imposing a prior based on the REXCESS sample on $\alpha$ reduces these problems significantly. However, it is clear from Fig. 29 that in some cases these spurious constraints still do occur, particularly in $\alpha$ for small angular-size clusters, and $\beta$ for medium angular-size clusters. Surprisingly, $\beta$ is often recovered correctly for large angular-size clusters - this is due to the intersection of the physically motivated prior on $\theta_{\mathrm{s}}$ and $Y_{\text {tot }}$ and the degeneracy direction between $\theta_{\mathrm{s}}$ and $\beta$.

It is also clear from Fig. 29 that varying the shape parameters does not aid in recovering the correct $\theta_{\mathrm{s}}$ and $Y_{\text {tot }}$ values for large angular-size clusters; joint analysis of Planck and AMI data is required to achieve this. As a first approximation, using a Planck-derived prior on $Y_{\text {tot }}$ can help, but does not improve the constraints on $\alpha$ and $\beta$.

It is also interesting to note that our parameter constraints are not very reliant on noise level. Our initial tests were made on simulated data with unrealistically small noise levels of $100 \mu \mathrm{Jy}$ per visibility; when we moved to simulations with more realistic noise levels (of $\approx 120 \mu \mathrm{Jy}_{\text {beam }}{ }^{-1}$ across the channel-averaged map), the constraints changed very little. As long as one has a good detection, it seems that the limiting factor on our parameter constraints is very much the range of angular scales present in the data with respect to the size of the cluster, rather than the detection significance.

\subsection{Analysis of real data}

For all the clear detections in the sample, we re-extract the cluster parameters allowing $\alpha$ and $\beta$ to vary as described in Sect. 5.1. The constraints on $Y_{\text {tot }}$ and $\theta_{\mathrm{s}}$ are on the whole broader but the positions of the maxima are unchanged. The full twodimensional constraints for the whole sample are available online ${ }^{8}$; here we present a few examples.

\subsubsection{Abell 1413 (PSZ1 G226.19+76.78)}

Abell 1413 is well-detected by AMI, with an evidence ratio of $\Delta \ln (\mathcal{Z})=26$, and Planck, with a PwS S/N of 9.8 and detections by all three algorithms. It is at redshift $z=0.143$ (e.g. Struble $\&$ Rood 1987) so could be expected to have a large angular size; the $\theta_{500}$ value inferred from the X-ray luminosity is $\approx 7.9$ arcmin (Böhringer et al. 2000; Piffaretti et al. 2011), corresponding to $\theta_{\mathrm{s}} \approx 6.7$ arcmin for $c_{500}=1.177$. The AMI constraints on $\theta_{\mathrm{s}}$ and $Y_{\text {tot }}$ could therefore be expected to be biased downward if the profile differs from the universal profile. Indeed, the Planck constraints indicate much higher values of both (see Fig. B.1 under the Planck name of PSZ1 G226.19+76.78). From the simulation results we can therefore expect to produce some constraints on $\alpha$ and $\beta$ from the AMI data, although not to recover the correct values of $\theta_{\mathrm{s}}$ and $Y_{\text {tot }}$; the posterior distributions for the real data are shown in Fig. 32.

In Planck Collaboration Int. V (2013), Planck and XMMNewton data were used to produce fitted values for $\alpha$ and $\beta$ for a sample of high-S/N Planck clusters. The sample includes seven of the clear detections in our SZ sample (however we note that for three of these, Planck Collaboration Int. V 2013 report non-physical values for $(\gamma, \alpha, \beta)$ producing negative values of $Y_{\text {tot }}$ because of the $\Gamma$ functions in Eq. (3)). Their reported values for Abell 1413 are $\alpha=0.83$ and $\beta=4.31$ ( $\gamma$ fixed at 0.31 ), which are plotted for comparison in Fig. 32. The AMI analysis produces a somewhat higher (but consistent) value for $\alpha$; although the Planck $\beta$ estimate is outside our prior range for $\beta$, our analysis shows no tendency to push toward the lower limit, toward the Planck value. However, assuming the shape of the Planck $\alpha-\beta$ degeneracy for the individual clusters is similar to that for their stacked profile (reproduced in Fig. 33), the AMI and Planck constraints on $\beta$ could be consistent.

\subsubsection{RXC J2228.6+2036 (PSZ1 G083.30-31.01)}

Similarly to Abell 1413, RXC J2228.6+2036 is well-detected by AMI $(\Delta \ln (\mathcal{Z})=28)$ and Planck $(S / N=7.3$, detected by all algorithms). It is at higher redshift, $z=0.412$ (Böhringer et al. 2000) so the large value for $\theta_{\mathrm{s}}$ of $\approx 4.5 \mathrm{arcmin}$ preferred by Planck is slightly surprising. Figure 34 shows the posteriors on $\theta_{\mathrm{s}}$ and $Y_{\text {tot }}$ produced by AMI and Planck, as well as the region of the space predicted by the physical model described in Olamaie et al. (2012) assuming the universal pressure profile for the gas, and the Tinker mass function (Tinker et al. 2008). The AMI posterior is much more consistent with the prediction than the Planck posterior; also our simulations have shown that if the correct value were $\theta_{\mathrm{s}} \approx 4.5$, we should recover it even if the profile deviates from the universal profile. In addition, $\theta_{500}$ determined from the X-ray luminosity is 3.9 arcmin (Böhringer et al. 2000; Piffaretti et al. 2011), corresponding to $\theta_{\mathrm{s}}=3.3 \mathrm{arcmin}$ for $c_{500}=1.177$ is consistent with the AMI

8 http://www.astro.phy.cam.ac.uk/surveys/ami-planck/ 

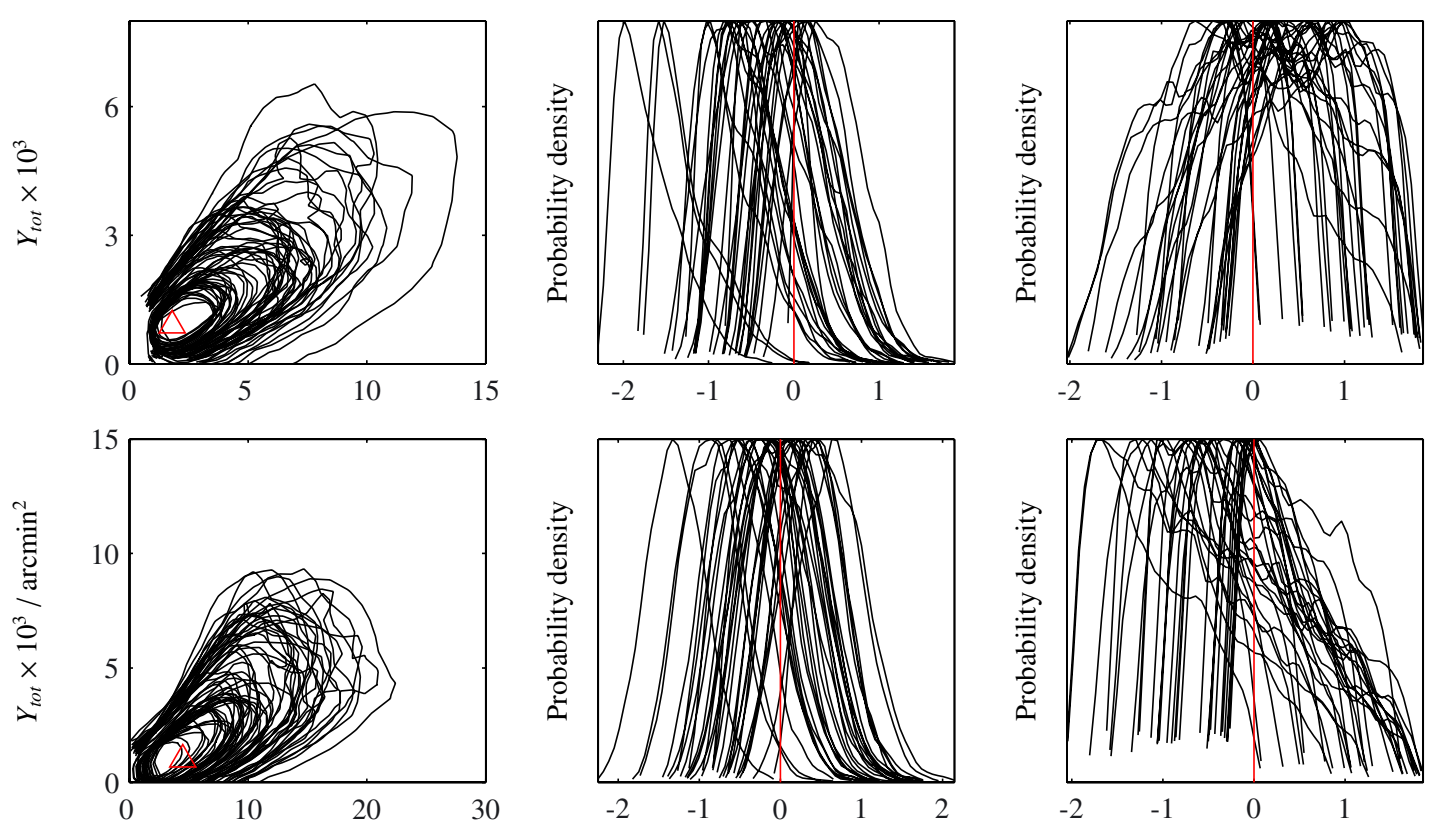

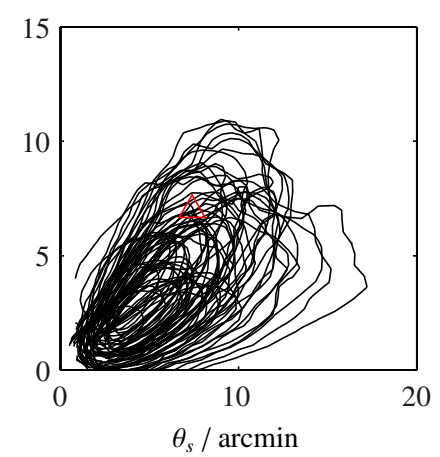

(a)

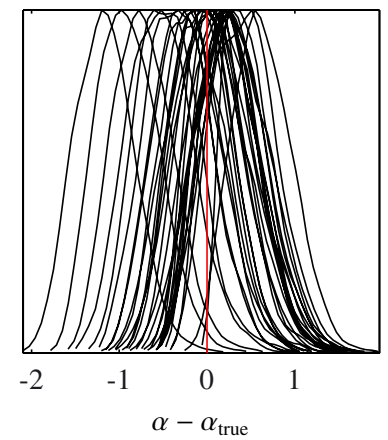

(b)

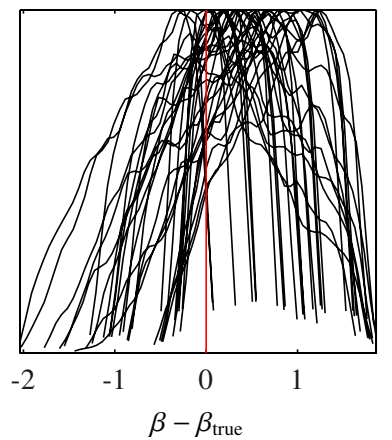

(c)

Fig. 29. Posterior distributions for simulated clusters with realistic noise levels (see text for details), and varying GNFW shape parameter values based on the REXCESS sample (Böhringer et al. 2007; Arnaud et al. 2010). a) shows the two-dimensional $\theta_{\mathrm{s}}$ and $Y_{\text {tot }}$ posterior, and b) and c) show the one-dimensional posteriors for $\alpha$ and $\beta$, shifted to be centred on the appropriate true value. In all cases $\gamma$ is fixed to the universal value, $\alpha$ has a truncated Gaussian prior based on the REXCESS sample, $\beta$ is varied uniformly between 4.5 and 6.5 , and the joint two-dimensional prior on $Y_{\text {tot }}$ and $\theta_{\mathrm{s}}$ is used. Results for three different angular sizes are shown (from top to bottom, $\theta_{\mathrm{s}}=1.8,4.5$ and 7.4 arcmin); the input parameter values are marked with red triangles and lines.

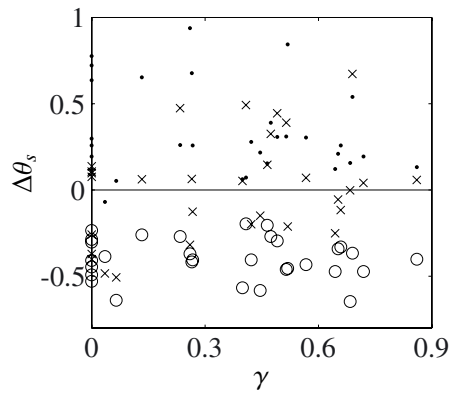

(a) (b)

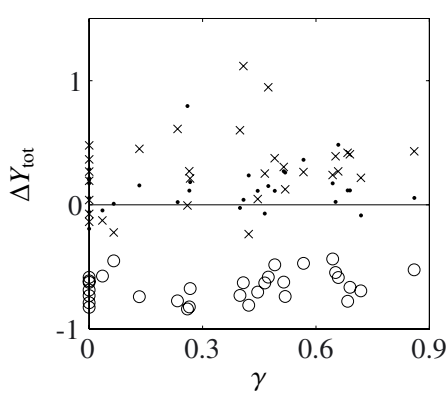

Fig. 30. Fractional difference [(MAP value - true value)/(true value)] in $\theta_{\mathrm{s}}$ a) and $\left.Y_{\mathrm{tot}} \mathbf{b}\right)$ as a function of the input value of $\gamma$. Clusters with $\theta_{\mathrm{s}}=1.8$ are plotted as dots, $\theta_{\mathrm{s}}=4.5$ as crosses and $\theta_{\mathrm{s}}=7.4$ as open circles.

mean value of $2.3 \mathrm{arcmin}$. We therefore conclude that in this case the Planck $\theta_{\mathrm{s}}$ estimate is likely to be an over-estimate.
Figure 34 also shows the posteriors on $\alpha$ and $\beta$ resulting from the AMI analysis. Assuming the AMI value of $\theta_{\mathrm{s}}$ is correct, we should be able to produce some constraint on $\beta$; indeed, there is a weak preference for higher values of $\beta$, while the posterior distribution for $\alpha$ mostly recovers the prior. The fitted $\alpha$ and $\beta$ values from Planck Collaboration Int. V (2013) are also shown and in this case are very consistent with the AMI constraints.

\subsection{PSZ1 G134.31-06.57}

PSZ1 G134.31-06.57 is a new Planck cluster at unknown redshift, with Planck $S / N=5.4$ and AMI $\Delta \ln (\mathcal{Z})=31$. The Planck and AMI constraints for this cluster overlap, and the different degeneracy directions result in a considerably tighter joint constraint, giving $\theta_{\mathrm{s}} \approx 4.5 \mathrm{arcmin}$ (see Fig. B.1). At this angular size, AMI data should produce constraints on $\alpha$. Figure 35 shows the parameter constraints $-\alpha$ moves away from the prior to a higher value of $\approx 1.5$, while $\beta$ also shows a weak constraint to values higher than the universal value. 

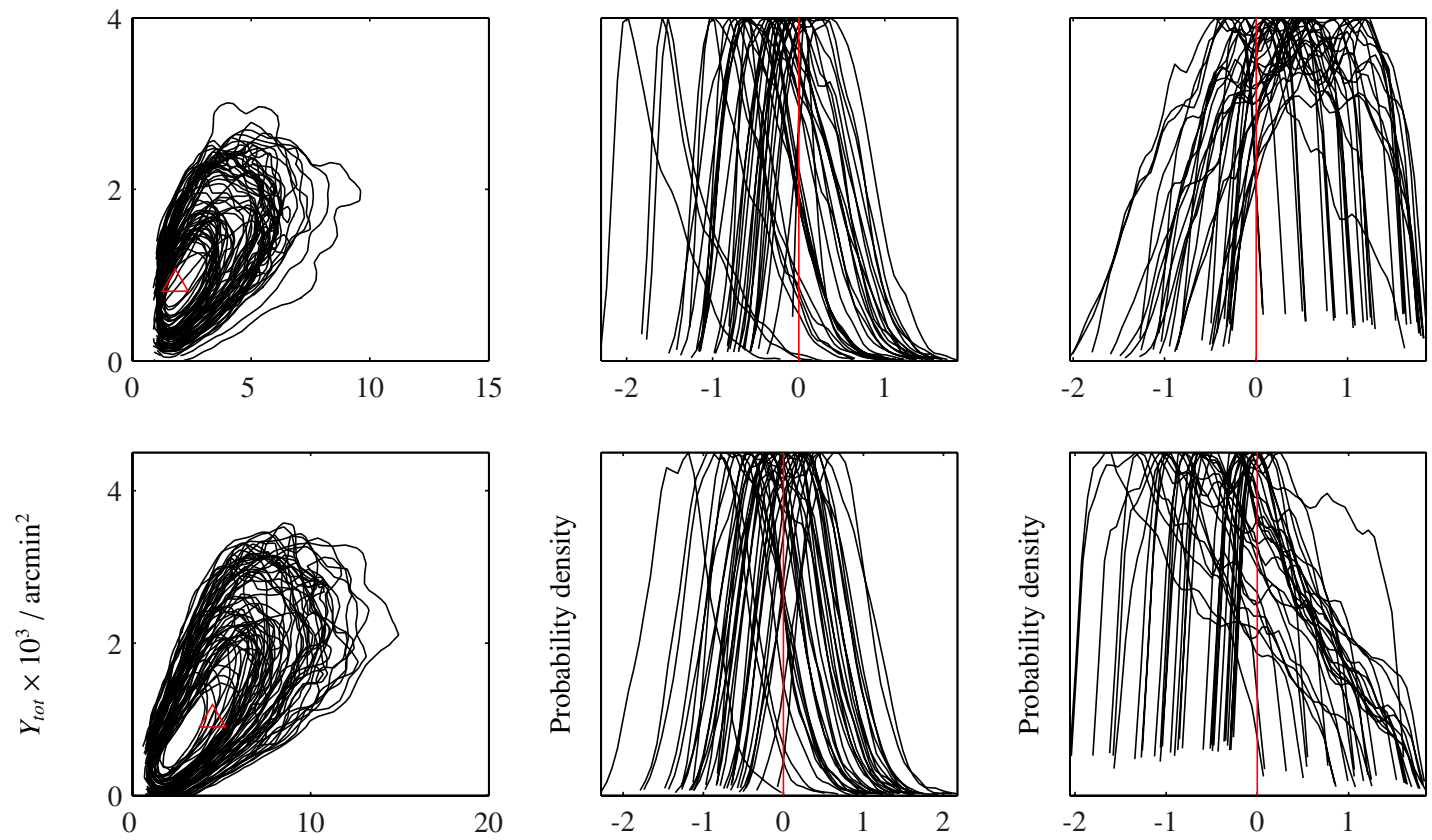

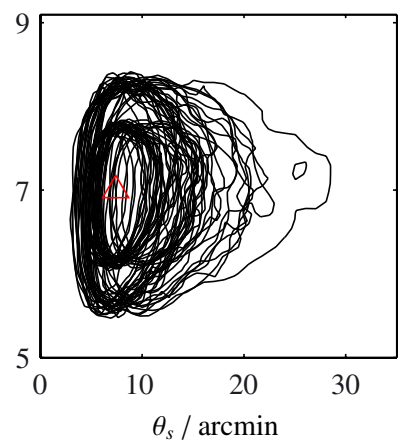

(a)

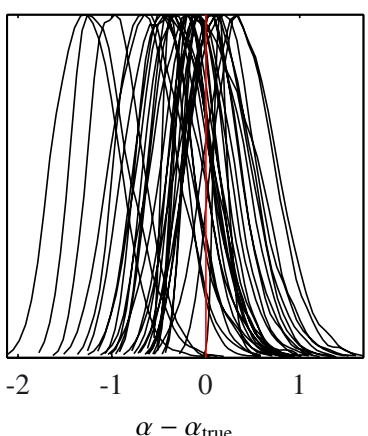

(b)

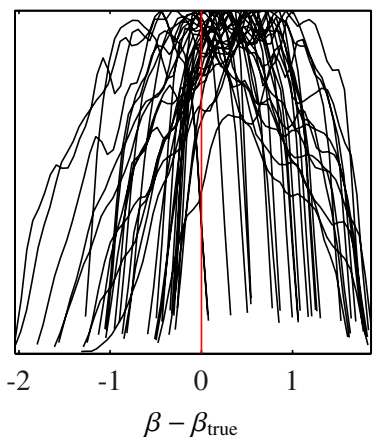

(c)

Fig. 31. Posterior distributions for simulated clusters with realistic noise levels (see text for details), and varying GNFW shape parameter values based on the REXCESS sample (Böhringer et al. 2007; Arnaud et al. 2010). a) shows the two-dimensional $\theta_{\mathrm{s}}$ and $Y_{\text {tot }}$ posterior, and b) and c) show the one-dimensional posteriors for $\alpha$ and $\beta$, shifted to be centred on the appropriate true value. In all cases $\gamma$ is fixed to the universal value, $\alpha$ has a truncated Gaussian prior based on the REXCESS sample, $\beta$ is varied uniformly between 4.5 and 6.5, a Planck-like Gaussian prior is used on $Y_{\mathrm{tot}}$ and $\theta_{\mathrm{S}}$ has the conditional prior drawn from the two-dimensional prior. Results for three different angular sizes are shown (from top to bottom, $\theta_{\mathrm{s}}=1.8,4.5$ and 7.4$)$; the input parameter values are marked with red triangles and lines.

\subsubsection{Properties of $\alpha$ and $\beta$ in the sample}

Figure 36 shows a histogram of the recovered mean $\alpha$ and $\beta$ values for all the clear detections in the sample.

Planck Collaboration Int. V (2013) and Sayers et al. (2013) both derive average pressure profiles for smaller samples of clusters using SZ data from Planck and BOLOCAM respectively. In both analyses, the radial profiles derived from the SZ maps are scaled by X-ray-determined $r_{500}$ values and then stacked; a GNFW model is fitted to the stacked profiles (+ X-ray points for the inner part of the profile in Planck Collaboration Int. V 2013). Their final best fit parameters are given by $\left(c_{500}, \gamma, \alpha, \beta\right)=$ $(1.81,0.31,1.33,4.1)$ and $(1.18,0.67,0.86,3.67)$ respectively. In contrast, the AMI analysis does not rely on X-ray estimates of $r_{500}$, being based purely on the AMI SZ data. The AMI preferred values for $\beta$ are on the whole centred around the universal value predicted by simulations, and do not show a trend towards the lower values derived from the Planck and BOLOCAM analyses. The AMI mean $\alpha$ estimates show a slight trend toward higher values, in agreement with the Planck value and in disagreement with the BOLOCAM value. However, since there are large (and different) degeneracies between the GNFW model parameters in the three analyses it is difficult to judge whether the analyses truly disagree (see Fig. 33, where it is clear that higher $\beta$ values are not ruled out by the Planck likelihood).

The AMI data for these clusters therefore indicate that although individual clusters do not necessarily conform to the universal profile and it is important to take this into account when analysing AMI data, on the whole the average profile for the sample remains close to the universal profile shape even though these clusters are selected via a very different selection function compared to the REXCESS sample.

\section{Conclusions}

We have followed up the 195 clusters from the Planck union catalogue that are visible to AMI, lie at $z>0.100$ and have Planck $S / N \geq 5$. Of these, we reject 72 due to difficult radio 

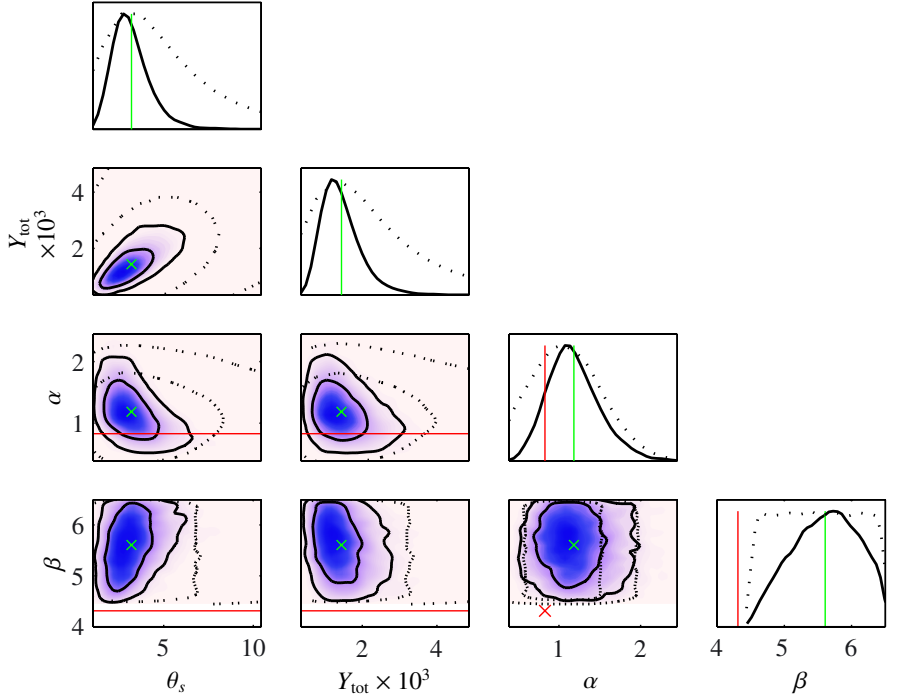

Fig. 32. AMI posterior distributions for A1413, allowing $\alpha$ and $\beta$ to vary. Posterior means are indicated with green lines and crosses, and the Planck + XMM-Newton estimates of $\alpha$ and $\beta$ from Planck Collaboration Int. V (2013) are shown with red lines and crosses. The priors on the parameters in the AMI analysis are shown as black dashed lines. $\theta_{\mathrm{s}}$ is in arcmin and $Y_{\text {tot }}$ is in $\operatorname{arcmin}^{2}$.
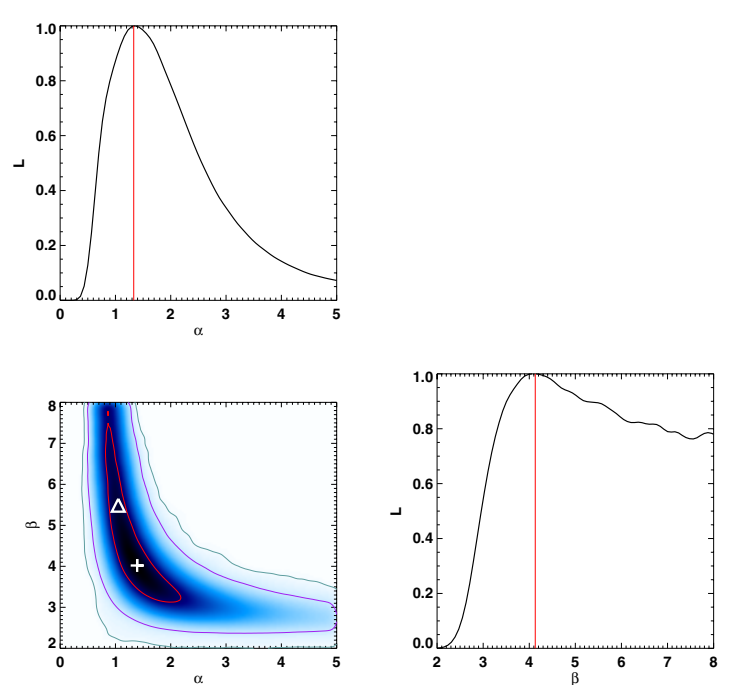

Fig. 33. Marginalised posterior likelihood distribution for $\alpha$ and $\beta$ based on stacked Planck and XMM-Newton data for a sample of high-S/N Planck clusters (from Planck Collaboration Int. V 2013). The white cross marks the position of the best-fit value, and the white triangle marks the universal values.

source environment, leaving a total SZ sample of 123 . We find that:

1. We detect 99 of the clusters, including 79 very good detections.

2. We re-confirm 14 of 16 new clusters already confirmed by other observations, and validate 14 of 25 new clusters which were not confirmed at the time the Planck catalogue was published.

3. We do not detect 24 of the clusters, which may be too extended for AMI to detect, be significantly offset from the phase centre, have a gas pressure profile deviating significantly from the universal profile, or be spurious detections
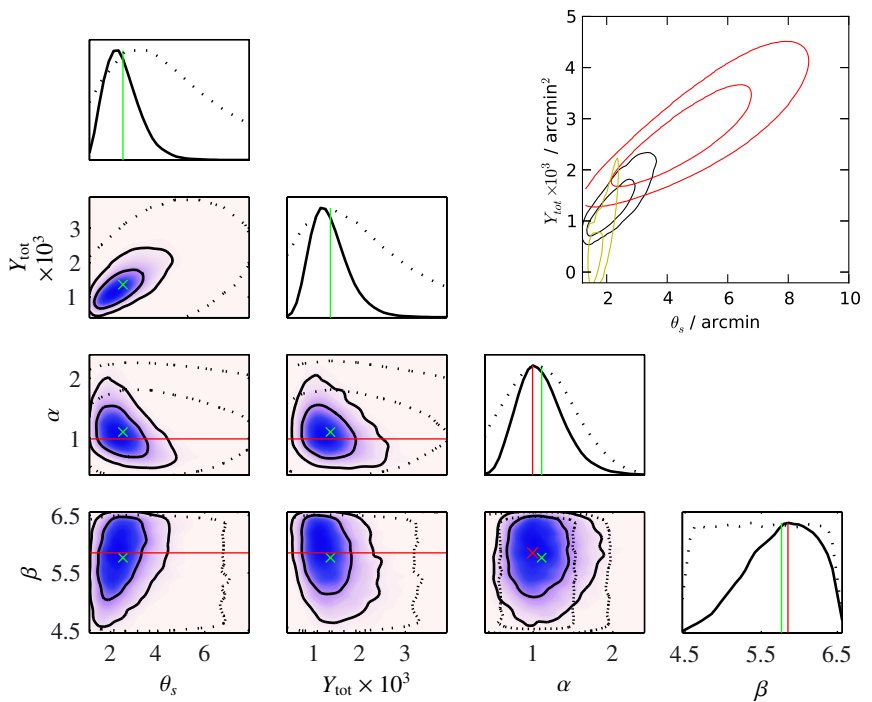

Fig. 34. AMI posterior distributions for RXC J2228.6+2036, allowing $\alpha$ and $\beta$ to vary. Posterior means are indicated with green lines and crosses, and the Planck values for $\alpha$ and $\beta$ from Planck Collaboration Int. V (2013) are shown with red lines and crosses. The priors on the parameters in the AMI analysis are shown as black dashed lines. $\theta_{\mathrm{s}}$ is in arcmin and $Y_{\text {tot }}$ is in $\operatorname{arcmin}^{2}$. Also shown in the upper right hand corner are the posteriors produced by AMI (black) and Planck (red) using the universal profile, and a prediction produced by the physical model described in Olamaie et al. (2012) based on a redshift of $z=$ 0.412 (yellow).
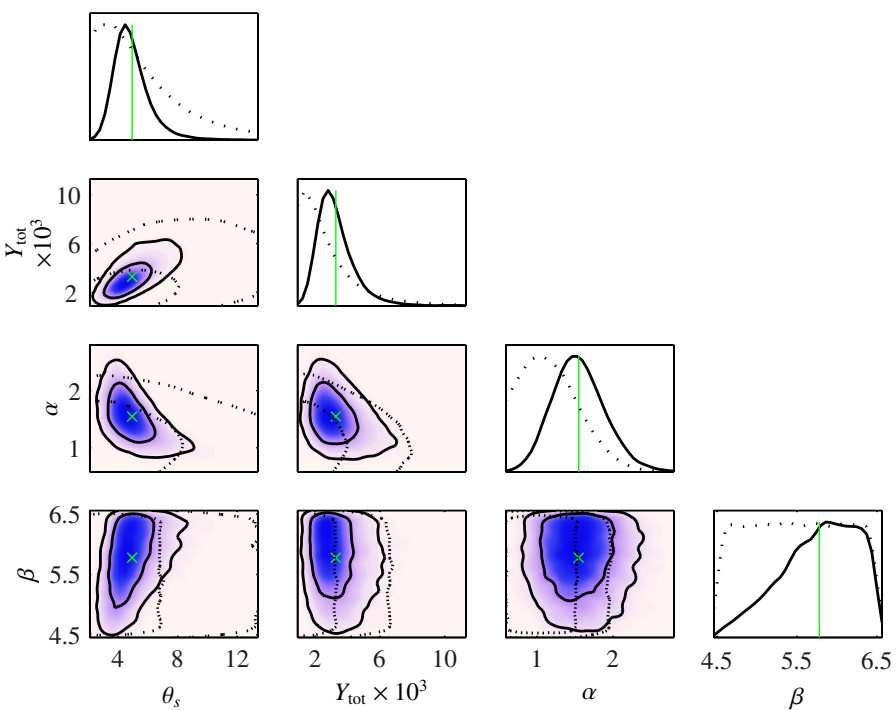

Fig. 35. AMI posterior distributions for PSZ1 G134.31-06.57, allowing $\alpha$ and $\beta$ to vary. Posterior means are indicated with green lines and crosses. The priors on the parameters in the AMI analysis are shown as black dashed lines. $\theta_{\mathrm{s}}$ is in arcmin and $Y_{\mathrm{tot}}$ is in $\operatorname{arcmin}^{2}$.

by Planck. $75 \%$ of the AMI non-detections are detected by $<3$ Planck algorithms, as opposed to $18 \%$ of the AMI detections; none of the AMI non-detections have quality flag values of 1 . These correlations indicate that an AMI nondetection is a good indicator for a spurious Planck detection.

4. Comparing the AMI positional estimates to those produced by $\mathrm{PwS}$ and the MMF algorithms shows that PwS positional estimates are generally more accurate, a more reliable 


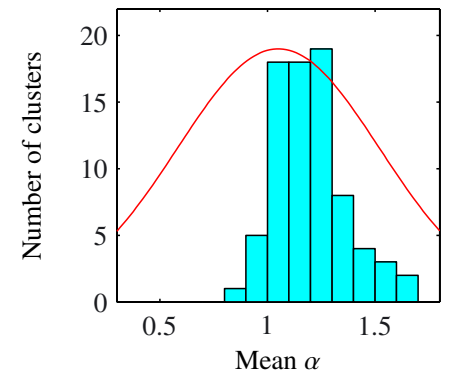

(a)

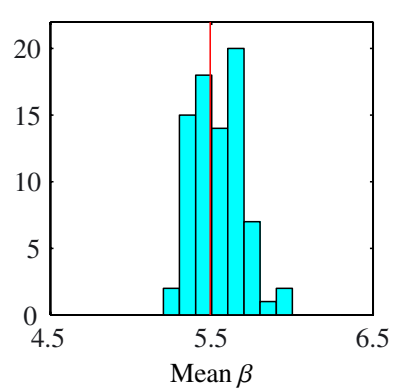

(b)
Fig. 36. The distribution of mean values of $\alpha$ and $\beta$ obtained for all the clear detections in the SZ sample. For comparison, the REXCESSbased prior on $\alpha$ (scaled arbitrarily) is also plotted in red, and the universal value of $\beta$ predicted from numerical simulations is indicated with a red line.

function of $\mathrm{S} / \mathrm{N}$, and have a positional error estimate consistent with the true uncertainty in the positions; in contrast, the MMF3 positional errors are over-estimated by a factor of $\approx 3$.

5. The trend seen in AP2013 where Planck consistently characterises clusters to be of larger angular size and brighter is continued in the larger sample, particularly for high Planck $\mathrm{S} / \mathrm{N}$ clusters; our simulation results suggest that this may be caused by deviation from the universal profile used for parameter recovery.

6. We can generalise the model used for parameter extraction from AMI data to consider variation in $\alpha$ and $\beta$, however the priors on the shape parameters must be considered carefully since degeneracies with $\theta_{\mathrm{s}}$ and $Y_{\text {tot }}$ can produce spurious onedimensional constraints on the shape parameters.

7. AMI data alone cannot reliably constrain $\theta_{\mathrm{s}}$ and $Y_{\text {tot }}$ for clusters of angular size $\theta_{\mathrm{s}} \gtrsim 5 \operatorname{arcmin}$ when there is uncertainty in the pressure profile of the cluster; it can however be used to constrain $\alpha$ and $\beta$.

8. AMI data can be used to constrain $\theta_{\mathrm{s}}, Y_{\text {tot }}$ and $\beta(\alpha)$ simultaneously for clusters of angular size $\approx 3 \operatorname{arcmin}(\approx 5 \operatorname{arcmin})$, given a careful choice of priors on $\alpha$ and $\beta$.

9. While deviation from the universal profile has been shown to be important for analysing AMI data on a cluster-by-cluster basis, overall the $\beta$ values obtained by re-analysing all of the clear detections from the Planck sample with varying $\alpha$ and $\beta$ do not show support for deviation from the universal $\beta$ value derived from numerical simulations.

Acknowledgements. We are grateful to a substitute referee who responded rapidly, with useful comments, to our paper. The AMI telescope is supported by Cambridge University. Y.C.P. and C.R. acknowledge support from CCT/Cavendish Laboratory and STFC studentships, respectively. Y.C.P. and M.O. acknowledge support from Research Fellowships from Trinity College and Sidney Sussex College, Cambridge, respectively. This work was partially undertaken on the COSMOS Shared Memory system at DAMTP, University of Cambridge operated on behalf of the STFC DiRAC HPC Facility. This equipment is funded by BIS National E-infrastructure capital grant ST/J005673/1 and STFC grants ST/H008586/1, ST/K00333X/1. C.M. acknowledges her KICC Fellowship grant funding for the procurement of the cluster used for computational work. In addition, we would like to thank the IOA computing support team for maintaining the cluster.

\section{References}

Abell, G. O. 1958, ApJS, 3, 211

Aghanim, N., da Silva, A. C., \& Nunes, N. J. 2009, A\&A, 496, 637 Albert, C. E., White, R. A., \& Morgan, W. W. 1977, ApJ, 211, 309 Allen, S. W., Edge, A. C., Fabian, A. C., et al. 1992, MNRAS, 259, 67 Angulo, R. E., Springel, V., White, S. D. M., et al. 2012, MNRAS, 426, 2046
Appenzeller, I., Thiering, I., Zickgraf, F.-J., et al. 1998, ApJS, 117, 319 Arnaud, M., Pratt, G. W., Piffaretti, R., et al. 2010, A\&A, 517, A92 Barker, R., Biddulph, P., Bly, D., et al. 2006, MNRAS, 369, L1

Birkinshaw, M., Gull, S. F., \& Northover, K. J. E. 1978, Nature, 275, 40

Birkinshaw, M., Gull, S. F., \& Hardebeck, H. 1984, Nature, 309, 34

Böhringer, H., Voges, W., Huchra, J. P., et al. 2000, ApJS, 129, 435

Böhringer, H., Schuecker, P., Pratt, G. W., et al. 2007, A\&A, 469, 363

Carvalho, P., Rocha, G., \& Hobson, M. P. 2009, MNRAS, 393, 681

Carvalho, P., Rocha, G., Hobson, M. P., \& Lasenby, A. 2012, MNRAS, 427, 1384

da Silva, A. C., Kay, S. T., Liddle, A. R., \& Thomas, P. A. 2004, MNRAS, 348, 1401

Davies, M. L., Franzen, T. M. O., Waldram, E. M., et al. 2011, MNRAS, 415, 2708

Ebeling, H., Edge, A. C., \& Henry, J. P. 2001, ApJ, 553, 668

Ebeling, H., Mullis, C. R., \& Tully, R. B. 2002, ApJ, 580, 774

Feroz, F., \& Hobson, M. P. 2008, MNRAS, 384, 449

Feroz, F., Hobson, M. P., \& Bridges, M. 2009a, MNRAS, 398, 1601

Feroz, F., Hobson, M. P., Zwart, J. T. L., Saunders, R. D. E., \& Grainge, K. J. B. 2009b, MNRAS, 398, 2049

Fetisova, T. S. 1981, Sov. Astron., 25, 647

Franzen, T. M. O., Davies, M. L., Waldram, E. M., et al. 2011, MNRAS, 415, 2699

Gower, J. F. R., Scott, P. F., \& Wills, D. 1967, MmRAS, 71, 49

Harrison, D., Sutton, D., Carvalho, P., \& Hobson, M. 2015, MNRAS, 451, 2610 Hurley-Walker, N., Brown, M. L., Davies, M. L., et al. 2011, MNRAS, 414, L75 Hurley-Walker, N., Bridle, S., Cypriano, E. S., et al. 2012, MNRAS, 419, 2921 Jeffreys, H. 1961, Theory of Probability, 3rd edn. (Oxford, England: Oxford) Jenkins, A., Frenk, C. S., White, S. D. M., et al. 2001, MNRAS, 321, 372 Jones, M., Saunders, R., Alexander, P., et al. 1993, Nature, 365, 320 Kay, S. T., Peel, M. W., Short, C. J., et al. 2012, MNRAS, 422, 1999 Kristian, J., Sandage, A., \& Westphal, J. A. 1978, ApJ, 221, 383 Mehrtens, N., Romer, A. K., Hilton, M., et al. 2012, MNRAS, 423, 1024

Melin, J., Bartlett, J. G., \& Delabrouille, J. 2006, A\&A, 459, 341

Melin, J.-B., Aghanim, N., Bartelmann, M., et al. 2012, A\&A, 548, A51

Motl, P. M., Hallman, E. J., Burns, J. O., \& Norman, M. L. 2005, ApJ, 623, L63

Nagai, D. 2006, ApJ, 650, 538

Nagai, D., Kravtsov, A. V., \& Vikhlinin, A. 2007, ApJ, 668, 1

Navarro, J. F., Frenk, C. S., \& White, S. D. M. 1997, ApJ, 490, 493

Olamaie, M., Hobson, M. P., \& Grainge, K. J. B. 2012, MNRAS, 423, 1534

Perley, R. A., \& Butler, B. J. 2013, ApJS, 204, 19

Petrov, L., Kovalev, Y. Y., Fomalont, E. B., \& Gordon, D. 2008, AJ, 136, 580

Piffaretti, R., Arnaud, M., Pratt, G. W., Pointecouteau, E., \& Melin, J.-B. 2011, A\&A, 534, A109

Planck and AMI Collaborations. 2013, A\&A, 550, A128

Planck Collaboration Int. V. 2013, A\&A, 550, A131

Planck Collaboration VIII. 2011, A\&A, 536, A8

Planck Collaboration X. 2011, A\&A, 536, A10

Planck Collaboration XX. 2014, A\&A, 571, A20

Planck Collaboration XXVIII. 2014, A\&A, 571, A28

Planck Collaboration XXIX. 2014, A\&A, 571, A29

Pravdo, S. H., \& Marshall, F. E. 1984, ApJ, 281, 570

Rodríguez-Gonzálvez, C., Shimwell, T. W., Davies, M. L., et al. 2012, MNRAS, 425,162

Rudy, D. J., Muhleman, D. O., Berge, G. L., Jakosky, B. M., \& Christensen, P. R. 1987, Icarus, 71, 159

Sayers, J., Czakon, N. G., Mantz, A., et al. 2013, ApJ, 768, 177

Shimwell, T. W., Rodríguez-Gonzálvez, C., Feroz, F., et al. 2013, MNRAS, 433, 2920

Struble, M. F., \& Rood, H. J. 1987, ApJS, 63, 543

Sunyaev, R. A., \& Zel'dovich, Y. B. 1972, Comments on Astrophysics and Space Physics, 4, 173

Tinker, J., Kravtsov, A. V., Klypin, A., et al. 2008, ApJ, 688, 709

Voges, W., Aschenbach, B., Boller, T., et al. 1999, A\&A, 349, 389

Wen, Z. L., Han, J. L., \& Liu, F. S. 2009, ApJS, 183, 197

Wen, Z. L., Han, J. L., \& Liu, F. S. 2012, ApJS, 199, 34

Zwart, J. T. L., Barker, R. W., Biddulph, P., et al. 2008, MNRAS, 391, 1545

Zwart, J. T. L., Feroz, F., Davies, M. L., et al. 2011, MNRAS, 418, 2754

Zwicky, F. 1937, ApJ, 86, 217

Zwicky, F., \& Kowal, C. T. 1968, Catalogue of Galaxies and of Clusters of Galaxies, Vol. VI

Zwicky, F., Herzog, E., Wild, P., Karpowicz, M., \& Kowal, C. T. 1961, Catalogue of galaxies and of clusters of galaxies, Vol. I

1 Academy of Sciences of Tatarstan, Bauman Str., 20, 420111 Kazan, Republic of Tatarstan, Russia 
2 Astrophysics Group, Cavendish Laboratory, University of Cambridge, J J Thomson Avenue, Cambridge CB3 OHE, UK e-mail: ycp21@mrao.cam.ac.uk

3 Atacama Large Millimeter/submillimeter Array, ALMA Santiago Central Offices, Alonso de Cordova 3107, Vitacura, Casilla 763 0355, Santiago, Chile

4 CNRS, IRAP, 9 Av. colonel Roche, BP 44346, 31028 Toulouse Cedex 4, France

5 CSIRO Astronomy \& Space Science, Australia Telescope National Facility, PO Box 76, Epping, NSW 1710, Australia

6 DSM/Irfu/SPP, CEA-Saclay, 91191 Gif-sur-Yvette Cedex, France

7 Department of Astronomy and Geodesy, Kazan Federal University, Kremlevskaya Str., 18, 420008 Kazan, Russia

8 Dpto. Astrofísica, Universidad de La Laguna (ULL), 38206 La Laguna, Tenerife, Spain

9 European Southern Observatory, ESO Vitacura, Alonso de Cordova 3107, Vitacura, Casilla 19001, Santiago, Chile

10 INAF-Osservatorio Astronomico di Roma, via di Frascati 33, 00040 Monte Porzio Catone, Italy

11 Institut d'Astrophysique Spatiale, CNRS (UMR 8617) Université Paris-Sud 11, Bâtiment 121, 91405 Orsay, France

12 Institute of Astronomy, University of Cambridge, Madingley Road, Cambridge CB3 OHA, UK

13 Institute of Theoretical Astrophysics, University of Oslo, Blindern, 0371 Oslo, Norway

14 Instituto de Astrofísica de Canarias, C/Vía Láctea s/n, 38205 La Laguna, Tenerife, Spain
15 Jodrell Bank Centre for Astrophysics, Alan Turing Building, School of Physics and Astronomy, The University of Manchester, Oxford Road, Manchester, M13 9PL, UK

16 Kavli Institute for Cosmology Cambridge, Madingley Road, Cambridge, CB3 OHA, UK

17 Laboratoire AIM, IRFU/Service d'Astrophysique - CEA/DSM CNRS - Université Paris Diderot, Bât. 709, CEA-Saclay, 91191 Gif-sur-Yvette Cedex, France

18 Laboratoire de Physique Subatomique et de Cosmologie, Université Joseph Fourier Grenoble I, CNRS/IN2P3, Institut National Polytechnique de Grenoble, 53 rue des Martyrs, 38026 Grenoble Cedex, France

19 Max-Planck-Institut für Extraterrestrische Physik, Giessenbachstraße, 85748 Garching, Germany

${ }^{20}$ Moscow Institute of Physics and Technology, Dolgoprudny, Institutsky per., 9, 141700 Moscow, Russia

21 Space Research Institute (IKI), Russian Academy of Sciences, Profsoyuznaya Str, 84/32, 117997 Moscow, Russia

22 Tübitak National Observatory, Akdeniz University Campus, 07058 Antalya, Turkey

${ }^{23}$ Universidad Andrés Bello, Dpto. de Ciencias Físicas, Facultad de Ciencias Exactas, Astronomía, Campus Casona de Las Condes, Fernández Concha 700, 7591538 Santiago/Las Condes, Chile

${ }^{24}$ Université de Toulouse, UPS-OMP, IRAP, 31028 Toulouse Cedex 4, France 
Y. C. Perrott et al.: Planck and AMI SZ measurements for 99 galaxy clusters

\section{Appendix A: Results table}

Table A.1. Summary of results for all clusters between $20^{\circ} \leq \delta<87^{\circ}$ with Planck $S / N>5$.

\begin{tabular}{|c|c|c|c|c|c|c|c|c|}
\hline Cluster name & $\begin{array}{c}\text { Planck } \\
S / N\end{array}$ & $\begin{array}{c}\text { Planck } \\
\text { det. }\end{array}$ & Category & $\Delta \ln (\mathcal{Z})$ & Aliases & $\begin{array}{c}\text { Previous } \\
\text { AMI }\end{array}$ & Redshift & Notes \\
\hline PSZ1 G075.71+13.51 & 25.96 & 111 & $\mathrm{LZ}$ & & $\begin{array}{l}\text { RXC J1921.1+4357, } \\
\text { A2319 }\end{array}$ & & 0.0557 & \\
\hline PSZ1 G110.99+31.74 & 22.70 & 111 & $\mathrm{LZ}$ & & $\begin{array}{l}\text { RXC J1703.8+7838, } \\
\text { A2256 }\end{array}$ & & 0.0581 & \\
\hline PSZ1 G044.24+48.66 & 19.56 & 111 & $\mathrm{LZ}$ & & $\begin{array}{l}\text { RXC J1558.3+2713, } \\
\text { A2142 }\end{array}$ & & 0.0894 & \\
\hline PSZ1 G072.61+41.47 & 19.42 & 111 & $\mathrm{R}$ & & $\begin{array}{l}\text { RXC J1640.3+4642, } \\
\text { A2219 }\end{array}$ & & 0.228 & \\
\hline PSZ1 G093.93+34.92 & 18.07 & 111 & $\mathrm{LZ}$ & & $\begin{array}{l}\text { RXC J1712.7+6403, } \\
\text { A2255 }\end{array}$ & & 0.0809 & \\
\hline PSZ1 G097.72+38.13 & 17.21 & 111 & $\mathrm{Y}$ & 33.77 & $\begin{array}{l}\text { RXC J1635.8+6612, } \\
\text { A2218 }\end{array}$ & $2,5,6$ & 0.1709 & \\
\hline PSZ1 G186.37+37.26 & 15.51 & 111 & $\mathrm{R}$ & & $\begin{array}{l}\text { RXC J0842.9+3621, } \\
\text { A697 }\end{array}$ & & 0.282 & \\
\hline PSZ1 G057.84+87.98 & 15.25 & 111 & $\mathrm{LZ}$ & & $\begin{array}{l}\text { RXC J1259.7+2756, } \\
\text { Coma, A1656 }\end{array}$ & & 0.0231 & \\
\hline PSZ1 G086.47+15.31 & 14.97 & 111 & $\mathrm{Y}$ & 14.58 & RXC J1938.3+5409 & & 0.26 & \\
\hline PSZ1 G033.84+77.17 & 14.20 & 111 & $\mathrm{LZ}$ & & $\begin{array}{l}\text { RXC J1348.8+2635, } \\
\text { A1795 }\end{array}$ & & 0.0622 & \\
\hline PSZ1 G170.22+09.74 & 14.12 & 111 & $\mathrm{R}$ & & 1RXS J060313.4+42 & 1231 & & \\
\hline PSZ1 G149.21+54.17 & 13.60 & 111 & $\mathrm{R}$ & & $\begin{array}{l}\text { RXC J1058.4+5647, } \\
\text { A1132 }\end{array}$ & & 0.1369 & \\
\hline PSZ1 G092.67+73.44 & 13.41 & 111 & $\mathrm{R}$ & & $\begin{array}{l}\text { RXC J1335.3+4059, } \\
\text { A1763 }\end{array}$ & & 0.2279 & \\
\hline PSZ1 G072.78-18.70 & 13.09 & 111 & M & 1.78 & $\begin{array}{l}\mathrm{ZwCl} 2120+2256, \\
\mathrm{ZwCl} 8503\end{array}$ & & 0.143 & \\
\hline PSZ1 G149.75+34.68 & 12.97 & 111 & $\mathrm{Y}$ & 46.38 & $\begin{array}{l}\text { RXC J0830.9+6551, } \\
\text { A665 }\end{array}$ & & 0.1818 & \\
\hline PSZ1 G191.00+06.65 & 12.44 & 111 & $\mathrm{LZ}$ & & RXC J0635.0+2231 & & 0.068 & \\
\hline PSZ1 G058.29+18.57 & 11.78 & 111 & $\mathrm{LZ}$ & & $\begin{array}{l}\text { RXC J1825.3+3026, } \\
\text { CIZA J1825.3+3026 }\end{array}$ & & 0.065 & \\
\hline PSZ1 G067.19+67.44 & 11.76 & 111 & $\mathrm{Y}$ & 28.83 & $\begin{array}{l}\text { RXC J1426.0+3749, } \\
\text { A1914 }\end{array}$ & $1,2,4,6$ & 0.1712 & \\
\hline PSZ1 G107.14+65.29 & 11.20 & 111 & $\mathrm{R}$ & & $\begin{array}{l}\text { RXC J1332.7+5032, } \\
\text { A1758 }\end{array}$ & 5 & 0.2799 & \\
\hline PSZ1 G055.58+31.87 & 10.83 & 111 & $\mathrm{R}$ & & $\begin{array}{l}\text { RXC J1722.4+3208, } \\
\text { A2261 }\end{array}$ & & 0.224 & \\
\hline PSZ1 G062.94+43.69 & 10.78 & 111 & $\mathrm{LZ}$ & & $\begin{array}{l}\text { RXC J1628.6+3932, } \\
\text { A2199 }\end{array}$ & & 0.0299 & \\
\hline PSZ1 G042.85+56.63 & 10.67 & 111 & $\mathrm{LZ}$ & & $\begin{array}{l}\text { RXC J1522.4+2742, } \\
\text { A2065 }\end{array}$ & & 0.0723 & \\
\hline PSZ1 G094.00+27.41 & 10.56 & 111 & $\mathrm{R}$ & & $\mathrm{H} 1821+643$ & & 0.3315 & \\
\hline
\end{tabular}

Notes. The rejection reason $(\mathrm{LZ}=$ low redshift, $\mathrm{R}=$ rejected by automated point-source criteria, $\mathrm{SE}=$ rejected manually for difficult source environment) or detection category $(\mathrm{Y}=$ clear detection, $\mathrm{M}=$ moderate detection, $\mathrm{N}=$ non-detection, $\mathrm{NN}=$ clear non-detection) is given in each case. Also given is the Planck S/N and the pipelines detecting the cluster (e.g. 110 indicates that the cluster was detected by MMF3, MMF1 but not PWS). Redshifts are taken from the Planck 2013 SZ catalogue. Some aliases for previously-known clusters are given from: Zwicky (1937, and references therein), Abell (1958), Zwicky et al. (1961), Gower et al. (1967), Albert et al. (1977), Fetisova (1981), Pravdo \& Marshall (1984), Appenzeller et al. (1998), Voges et al. (1999), Ebeling et al. (2001), Ebeling et al. (2002), Wen et al. (2009), Piffaretti et al. (2011, and references therein), Mehrtens et al. (2012), Wen et al. (2012), Planck Collaboration VIII (2011). Reference numbers refer to previously published AMI analyses, (1) Barker et al. (2006); (2) Hurley-Walker et al. (2011); (3) Zwart et al. (2011); (4) Hurley-Walker et al. (2012); (5) RodríguezGonzálvez et al. (2012); (6) AP2013; (7) Shimwell et al. (2013). $\Delta \ln (\mathcal{Z})$ is the Bayesian evidence difference. For non-detections, predicted $\mathrm{S} / \mathrm{N}$ ratios in the naturally-weighted $\left(\sigma_{\mathrm{NW}}\right)$ and $u v$-tapered $\left(\sigma_{\text {tap }}\right)$ maps are also given based on the Planck mean posterior parameter values. 
Table A.1. continued.

\begin{tabular}{|c|c|c|c|c|c|c|c|c|}
\hline Cluster name & $\begin{array}{c}\text { Planck } \\
S / N\end{array}$ & $\begin{array}{c}\text { Planck } \\
\text { det. }\end{array}$ & Category & $\Delta \ln (\mathcal{Z})$ & Aliases & $\begin{array}{c}\text { Previous } \\
\text { AMI }\end{array}$ & Redshift & Notes \\
\hline PSZ1 G180.25+21.03 & 10.54 & 111 & $\mathrm{R}$ & & $\begin{array}{l}\text { RXC J0717.5+3745, } \\
\text { MCS J0717.5+3745 }\end{array}$ & 2 & 0.546 & \\
\hline PSZ1 G053.52+59.52 & 10.46 & 111 & $\mathrm{Y}$ & 31.24 & $\begin{array}{l}\text { RXC J1510.1+3330, } \\
\text { A2034 }\end{array}$ & 6 & 0.113 & \\
\hline PSZ1 G125.34-08.65 & 10.22 & 111 & $\mathrm{Y}$ & 12.26 & $\begin{array}{l}\text { RXC J0107.7+5408, } \\
\text { ZwCl 0104+5350 }\end{array}$ & & 0.1066 & \\
\hline PSZ1 G124.20-36.47 & 10.13 & 111 & $\mathrm{R}$ & & $\begin{array}{l}\text { RXC J0055.9+2622, } \\
\text { A115 }\end{array}$ & 4 & 0.1971 & \\
\hline PSZ1 G112.48+57.02 & 9.97 & 111 & $\mathrm{LZ}$ & & $\begin{array}{l}\text { RXC J1336.1+5912, } \\
\text { A1767 }\end{array}$ & & 0.0701 & \\
\hline PSZ1 G049.22+30.84 & 9.90 & 111 & M & 1.61 & RXC J1720.1+2637 & 5 & 0.1644 & \\
\hline PSZ1 G226.19+76.78 & 9.79 & 111 & $\mathrm{Y}$ & 25.52 & $\begin{array}{l}\text { RXC J1155.3+2324, } \\
\text { A1413 }\end{array}$ & 5,6 & 0.1427 & \\
\hline PSZ1 G067.36+10.74 & 9.61 & 111 & $\mathrm{Y}$ & 10.47 & RXC J1916.1+3525 & & 0.209 & \\
\hline PSZ1 G056.79+36.30 & 9.58 & 111 & $\mathrm{LZ}$ & & $\begin{array}{l}\text { RXC J1702.7+3403, } \\
\text { A2244 }\end{array}$ & & 0.0953 & \\
\hline PSZ1 G084.47+12.63 & 9.54 & 111 & $\mathrm{Y}$ & 4.75 & RXC J1948.3+5113 & & 0.185 & \\
\hline PSZ1 G166.11+43.40 & 9.53 & 111 & $\mathrm{Y}$ & 27.21 & $\begin{array}{l}\text { RXC J0917.8+5143, } \\
\text { A773 }\end{array}$ & $2,5,6$ & 0.2172 & \\
\hline PSZ1 G139.17+56.37 & 9.48 & 111 & $\mathrm{R}$ & & $\begin{array}{l}\text { RXC J1142.5+5832, } \\
\text { A1351, } \\
\text { MCS J1142.4+5831 }\end{array}$ & & 0.322 & \\
\hline PSZ1 G167.64+17.63 & 9.43 & 111 & $\mathrm{Y}$ & 4.74 & $\begin{array}{l}\text { RXC J0638.1+4747, } \\
\text { ZwCl 0634+4750, } \\
\text { ZwCl } 1133\end{array}$ & & 0.174 & \\
\hline PSZ1 G057.63+34.92 & 9.03 & 111 & $\mathrm{LZ}$ & & $\begin{array}{l}\text { RXC J1709.8+3426, } \\
\text { A2249 }\end{array}$ & & 0.0802 & \\
\hline PSZ1 G113.84+44.33 & 8.98 & 111 & $\mathrm{Y}$ & 3.10 & $\begin{array}{l}\text { RXC J1414.2+7115, } \\
\text { A1895 }\end{array}$ & & 0.225 & \\
\hline PSZ1 G046.90+56.48 & 8.96 & 111 & M & 0.88 & $\begin{array}{l}\text { RXC J1524.1+2955, } \\
\text { A2069 }\end{array}$ & & 0.1145 & \\
\hline PSZ1 G077.89-26.62 & 8.74 & 111 & Y & 35.33 & $\begin{array}{l}\text { RXC J2200.8+2058, } \\
\text { A2409 }\end{array}$ & 5,6 & 0.147 & \\
\hline PSZ1 G139.61+24.20 & 8.66 & 111 & $\mathrm{Y}$ & 27.08 & & 6 & 0.2671 & \\
\hline PSZ1 G118.58+28.57 & 8.57 & 111 & $\mathrm{Y}$ & 4.83 & $\begin{array}{l}\text { RXC J1723.7+8553, } \\
\text { A2294 }\end{array}$ & & 0.178 & \\
\hline PSZ1 G071.21+28.86 & 8.46 & 011 & $\mathrm{Y}$ & 12.60 & $\begin{array}{l}\text { RXC J1752.0+4440, } \\
\text { MCS J1752.0+4440 }\end{array}$ & & 0.366 & \\
\hline PSZ1 G125.72+53.87 & 8.45 & 111 & $\mathrm{R}$ & & $\begin{array}{l}\text { RXC J1236.9+6311, } \\
\text { A1576, } \\
\text { MCS J1236.9+6311 }\end{array}$ & & 0.3019 & \\
\hline PSZ1 G098.12+30.30 & 8.45 & 111 & $\mathrm{LZ}$ & & $\begin{array}{l}\text { RXC J1754.6+6803, } \\
\text { ZwCl 1754+680 }\end{array}$ & & 0.077 & \\
\hline PSZ1 G165.06+54.13 & 8.44 & 111 & $\mathrm{Y}$ & 16.86 & $\begin{array}{l}\text { RXC J1023.6+4907, } \\
\text { A990 }\end{array}$ & 5,6 & 0.144 & \\
\hline PSZ1 G180.56+76.66 & 8.43 & 111 & $\mathrm{Y}$ & 7.13 & $\begin{array}{l}\text { RXC J1157.3+3336, } \\
\text { A1423 }\end{array}$ & 5 & 0.2138 & \\
\hline PSZ1 G048.08+57.17 & 8.36 & 101 & $\mathrm{LZ}$ & & $\begin{array}{l}\text { RXC J1521.2+3038, } \\
\text { A2061 }\end{array}$ & & 0.0777 & \\
\hline PSZ1 G157.32-26.77 & 8.35 & 111 & $\mathrm{Y}$ & 25.87 & $\begin{array}{l}\text { RXC J0308.9+2645, } \\
\text { MCS J0308.9+2645 }\end{array}$ & 2 & 0.356 & \\
\hline PSZ1 G163.69+53.52 & 8.26 & 111 & $\mathrm{Y}$ & 5.10 & $\begin{array}{l}\text { RXC J1022.5+5006, } \\
\text { A980 }\end{array}$ & & 0.158 & \\
\hline PSZ1 G157.44+30.34 & 8.19 & 011 & Y & 32.51 & $\begin{array}{l}\text { [ATZ98] B100, } \\
\text { RXC J0748.7+5941 }\end{array}$ & 6 & & \\
\hline
\end{tabular}


Y. C. Perrott et al.: Planck and AMI SZ measurements for 99 galaxy clusters

Table A.1. continued.

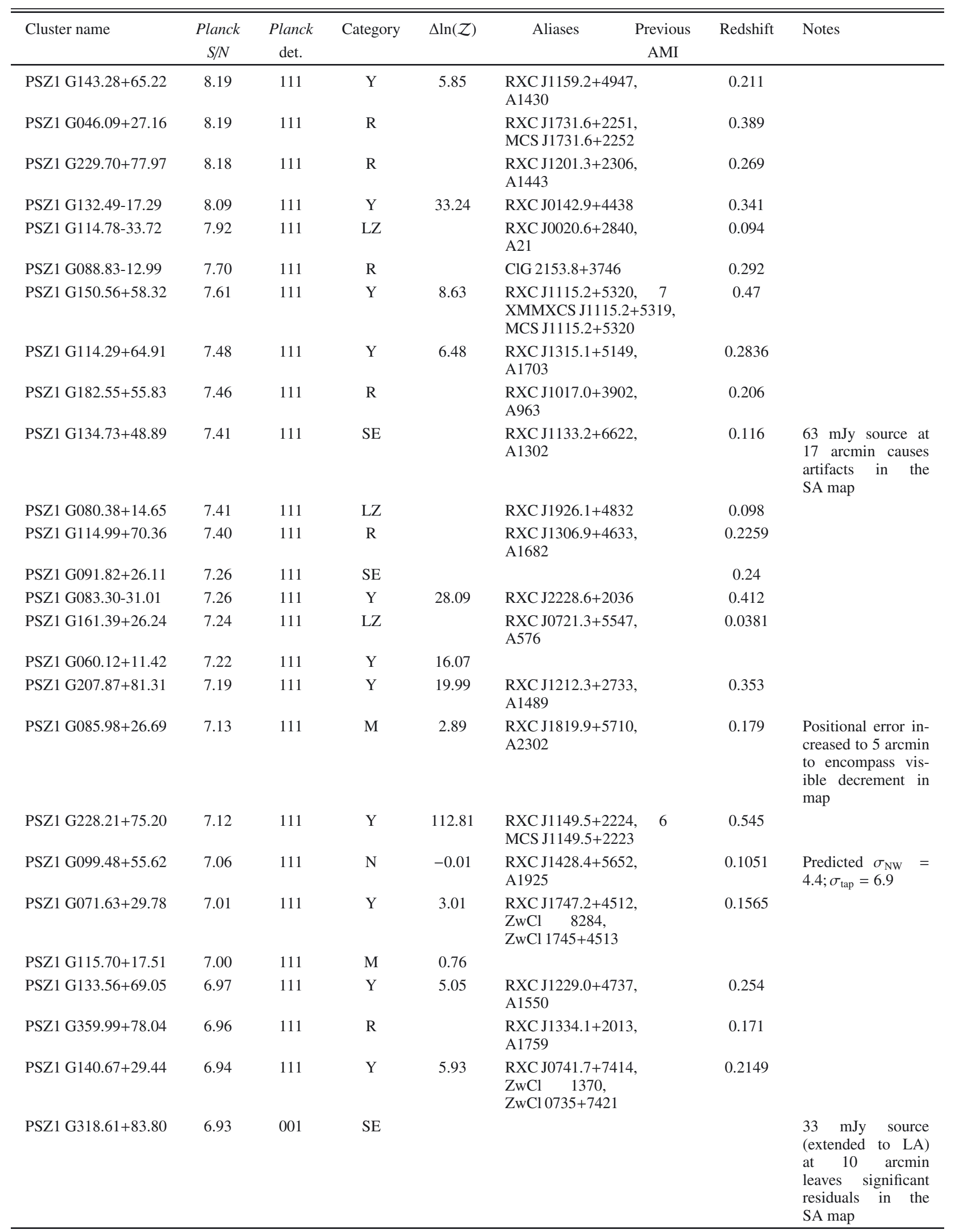


Table A.1. continued.

\begin{tabular}{|c|c|c|c|c|c|c|c|}
\hline Cluster name & $\begin{array}{c}\text { Planck } \\
S / N\end{array}$ & $\begin{array}{c}\text { Planck } \\
\text { det. }\end{array}$ & Category & $\Delta \ln (\mathcal{Z})$ & $\begin{array}{c}\text { Previous } \\
\text { AMI }\end{array}$ & Redshift & Notes \\
\hline PSZ1 G067.52+34.75 & 6.92 & 111 & $\mathrm{R}$ & & $\begin{array}{l}\text { RXC J1717.3+4226, } \\
\text { ZwCl 8193, } \\
\text { ZwCl 1715+4229 }\end{array}$ & 0.1754 & \\
\hline PSZ1 G113.26-29.69 & 6.91 & 111 & $\mathrm{R}$ & & $\begin{array}{l}\text { RXC J0011.7+3225, } \\
\text { A7 }\end{array}$ & 0.1073 & \\
\hline PSZ1 G098.85-07.27 & 6.89 & 011 & SE & & & & \\
\hline PSZ1 G096.89+24.17 & 6.89 & 111 & $\mathrm{Y}$ & 3.54 & $\begin{array}{l}\text { ZwCl 1856+6616, } \\
\text { PLCKESZ G096.87+24.21 }\end{array}$ & 0.3 & \\
\hline PSZ1 G138.60-10.85 & 6.86 & 111 & $\mathrm{Y}$ & 6.15 & & & \\
\hline PSZ1 G153.41+36.58 & 6.85 & 010 & $\mathrm{~N}$ & -2.70 & & & $\begin{array}{l}\text { Predicted } \sigma_{\mathrm{NW}} \\
3.0 ; \sigma_{\text {tap }}=3.7\end{array}$ \\
\hline PSZ1 G146.37-15.57 & 6.83 & 111 & $\mathrm{LZ}$ & & $\begin{array}{l}\text { RXC J0254.4+4134, } \\
\text { AWM7 }\end{array}$ & 0.0172 & \\
\hline PSZ1 G148.20+23.49 & 6.77 & 111 & $\mathrm{Y}$ & 3.19 & & & \\
\hline PSZ1 G121.09+57.02 & 6.72 & 111 & $\mathrm{Y}$ & 10.37 & 3,6 & 0.3436 & \\
\hline PSZ1 G118.46+39.31 & 6.67 & 111 & $\mathrm{Y}$ & 4.73 & RXC J1354.6+7715 & 0.3967 & \\
\hline PSZ1 G094.69+26.34 & 6.66 & 111 & $\mathrm{~N}$ & -0.26 & RXC J1832.5+6449 & 0.1623 & $\begin{array}{l}\text { Predicted } \sigma_{\mathrm{NW}} \\
4.1 ; \sigma_{\text {tap }}=5.3\end{array}$ \\
\hline PSZ1 G084.41-12.43 & 6.59 & 011 & $\mathrm{Y}$ & 15.82 & & & \\
\hline PSZ1 G102.97-04.77 & 6.56 & 011 & $\mathrm{Y}$ & 4.27 & & & \\
\hline PSZ1 G162.30-26.92 & 6.56 & 100 & $\mathrm{R}$ & & & & \\
\hline PSZ1 G109.14-28.02 & 6.56 & 111 & SE & & WHL J358.303+33.2696 & 0.4709 & \\
\hline PSZ1 G127.55+20.84 & 6.55 & 011 & $\mathrm{R}$ & & & & \\
\hline PSZ1 G100.18-29.68 & 6.54 & 111 & $\mathrm{R}$ & & & 0.485 & \\
\hline PSZ1 G049.35+44.36 & 6.53 & 111 & $\mathrm{LZ}$ & & $\begin{array}{l}\text { RXC J1620.5+2953, } \\
\text { A2175 }\end{array}$ & 0.0972 & \\
\hline PSZ1 G063.80+11.42 & 6.53 & 111 & $\mathrm{Y}$ & 3.78 & & & \\
\hline PSZ1 G098.96+24.87 & 6.52 & 111 & $\mathrm{LZ}$ & & RXC J1853.9+6822 & 0.0928 & \\
\hline PSZ1 G108.18-11.53 & 6.49 & 111 & $\mathrm{Y}$ & 16.62 & & & \\
\hline PSZ1 G066.41+27.03 & 6.48 & 111 & $\mathrm{Y}$ & 16.80 & WHL J269.219+40.1353 & 0.5699 & \\
\hline PSZ1 G100.16+41.66 & 6.43 & 111 & $\mathrm{R}$ & & $\begin{array}{l}\text { RXC J1556.1+6621, } \\
\text { A2146 }\end{array}$ & 0.2339 & \\
\hline PSZ1 G068.23+15.20 & 6.42 & 011 & $\mathrm{LZ}$ & & RXC J1857.6+3800 & 0.0567 & \\
\hline PSZ1 G166.61+42.12 & 6.38 & 111 & $\mathrm{Y}$ & 3.79 & $\begin{array}{l}\text { RXC J0909.3+5133, } \\
\text { A746 }\end{array}$ & 0.23225 & \\
\hline PSZ1 G099.84+58.45 & 6.35 & 111 & $\mathrm{Y}$ & 29.56 & WHL J213.697+54.7844 & 0.6305 & \\
\hline PSZ1 G054.99+53.42 & 6.31 & 111 & Y & 16.98 & $\begin{array}{l}\text { RXC J1539.7+3424, } 4,5 \\
\text { A2111 }\end{array}$ & 0.229 & \\
\hline PSZ1 G136.94+59.46 & 6.31 & 111 & $\mathrm{LZ}$ & & $\begin{array}{l}\text { RXC J1200.3+5613, } \\
\text { A1436 }\end{array}$ & 0.065 & \\
\hline PSZ1 G057.91+27.62 & 6.30 & 111 & $\mathrm{LZ}$ & & $\begin{array}{l}\text { RXC J1744.2+3259, } \\
\text { ZwCl } 8276\end{array}$ & 0.0757 & \\
\hline PSZ1 G105.25-17.96 & 6.29 & 111 & $\mathrm{R}$ & & RXC J2320.2+4146 & 0.14 & \\
\hline PSZ1 G195.60+44.03 & 6.27 & 111 & $\mathrm{R}$ & & $\begin{array}{l}\text { RXC J0920.4+3030, } \\
\text { A781 }\end{array}$ & 0.2952 & \\
\hline PSZ1 G068.32+81.81 & 6.27 & 111 & $\mathrm{SE}$ & & RXC J1322.8+3138 & 0.3083 & $\begin{array}{l}\text { Extended source to } \\
\text { south-east }\end{array}$ \\
\hline PSZ1 G118.88+52.40 & 6.25 & 111 & Y & 21.80 & $\begin{array}{l}\text { RXC J1314.4+6434, } \\
\text { A1704 }\end{array}$ & 0.22 & \\
\hline PSZ1 G186.98+38.66 & 6.22 & 111 & Y & 4.68 & $\begin{array}{l}\text { RXC J0850.2+3603, } \\
\text { ZwCl } 1953\end{array}$ & 0.378 & \\
\hline PSZ1 G083.62+85.08 & 6.17 & 111 & $\mathrm{R}$ & & $\begin{array}{l}\text { RXC J1305.9+3054, } \\
\text { A1677 }\end{array}$ & 0.1832 & \\
\hline
\end{tabular}


Y. C. Perrott et al.: Planck and AMI SZ measurements for 99 galaxy clusters

Table A.1. continued.

\begin{tabular}{|c|c|c|c|c|c|c|c|}
\hline Cluster name & $\begin{array}{l}\text { Planck } \\
\text { S/N }\end{array}$ & $\begin{array}{c}\text { Planck } \\
\text { det. }\end{array}$ & Category & $\Delta \ln (\mathcal{Z})$ & $\begin{array}{c}\text { Previous } \\
\text { AMI }\end{array}$ & Redshift & Notes \\
\hline PSZ1 G143.67+42.63 & 6.16 & 111 & $\mathrm{R}$ & & $\begin{array}{l}\text { RXC J1003.1+6709, } \\
\text { A910 }\end{array}$ & 0.206 & \\
\hline PSZ1 G192.19+56.12 & 6.14 & 111 & M & 0.22 & $\begin{array}{l}\text { RXC J1016.3+3338, } \\
\text { A961 }\end{array}$ & 0.124 & \\
\hline PSZ1 G135.03+36.03 & 6.12 & 111 & $\mathrm{Y}$ & 6.11 & $\begin{array}{l}\text { RXC J0947.2+7623, } \\
\text { MCS J0947.2+7623 }\end{array}$ & 0.345 & \\
\hline PSZ1 G074.75-24.59 & 6.10 & 111 & $\mathrm{~N}$ & -2.58 & $\mathrm{ZwCl} 2143+2014$ & 0.25 & $\begin{array}{l}\text { Predicted } \sigma_{\mathrm{NW}} \\
7.7 ; \sigma_{\text {tap }}=9.2\end{array}$ \\
\hline PSZ1 G152.68+25.43 & 6.10 & 111 & $\mathrm{LZ}$ & & $\begin{array}{l}\text { RXC J0704.4+6318, } \\
\text { A566 }\end{array}$ & 0.098 & \\
\hline PSZ1 G223.97+69.31 & 6.09 & 111 & M & 1.34 & $\begin{array}{l}\text { RXC J1123.9+2129, } \\
\text { A1246 }\end{array}$ & 0.1904 & \\
\hline PSZ1 G184.70+28.92 & 6.06 & 101 & $\mathrm{Y}$ & 20.64 & $\begin{array}{l}\text { RXC J0800.9+3602, 2, 4, } 5 \\
\text { A611 }\end{array}$ & 0.288 & \\
\hline PSZ1 G040.63+77.13 & 6.05 & 111 & $\mathrm{LZ}$ & & $\begin{array}{l}\text { RXC J1349.3+2806, } \\
\text { A1800 }\end{array}$ & 0.0748 & \\
\hline PSZ1 G131.02+29.98 & 6.02 & 111 & M & 2.98 & $\begin{array}{l}\text { RXC J0825.7+8218, } \\
\text { A625 }\end{array}$ & 0.2 & \\
\hline PSZ1 G171.01+39.44 & 6.01 & 111 & $\mathrm{Y}$ & 27.90 & & 0.5131 & \\
\hline PSZ1 G050.41+31.18 & 5.98 & 111 & $\mathrm{Y}$ & 10.30 & $\begin{array}{l}\text { RXC J1720.1+2740, } \\
\text { A2259 }\end{array}$ & 0.164 & \\
\hline PSZ1 G153.56+36.23 & 5.96 & 110 & M & 0.64 & & & \\
\hline PSZ1 G205.85+73.77 & 5.96 & 111 & $\mathrm{Y}$ & 17.84 & WHL J174.518+27.9773 & 0.4474 & \\
\hline PSZ1 G031.94+78.71 & 5.95 & 111 & $\mathrm{LZ}$ & & RXC J1341.8+2622 & 0.0724 & \\
\hline PSZ1 G187.53+21.92 & 5.88 & 111 & $\mathrm{Y}$ & 12.52 & $\begin{array}{l}\text { RXC J0732.3+3137, } \\
\text { A586 }\end{array}$ & 0.171 & \\
\hline PSZ1 G201.50+30.63 & 5.87 & 111 & $\mathrm{Y}$ & 15.32 & $\mathrm{ZwCl} 0824+2244$ & 0.287 & \\
\hline PSZ1 G096.87+52.48 & 5.85 & 111 & M & 1.25 & $\begin{array}{l}\text { RXC J1452.9+5802, } \\
\text { A1995 }\end{array}$ & 0.3179 & \\
\hline PSZ1 G078.67+20.06 & 5.84 & 011 & $\mathrm{R}$ & & & 0.45 & \\
\hline PSZ1 G040.06+74.94 & 5.84 & 111 & $\mathrm{LZ}$ & & $\begin{array}{l}\text { RXC J1359.2+2758, } \\
\text { A1831 }\end{array}$ & 0.0612 & \\
\hline PSZ1 G142.38+22.82 & 5.81 & 110 & $\mathrm{Y}$ & 7.22 & & & \\
\hline PSZ1 G142.17+37.28 & 5.79 & 100 & $\mathrm{NN}$ & -5.05 & & & $\begin{array}{l}\text { Predicted } \sigma_{\mathrm{NW}}= \\
6.5 ; \sigma_{\text {tap }}=8.6\end{array}$ \\
\hline PSZ1 G186.81+07.31 & 5.79 & 001 & $\mathrm{R}$ & & WHL J97.3409+26.5054 & 0.2577 & \\
\hline PSZ1 G105.91-38.39 & 5.77 & 111 & $\mathrm{Y}$ & 13.03 & & & $\begin{array}{l}\text { Positional uncer- } \\
\text { tainty increased } \\
\text { to } 5 \text { arcmin to } \\
\text { encompass large } \\
\text { decrement visible } \\
\text { in map }\end{array}$ \\
\hline PSZ1 G099.31+20.89 & 5.75 & 111 & $\mathrm{Y}$ & 7.41 & RXC J1935.3+6734 & 0.1706 & \\
\hline PSZ1 G137.56+53.88 & 5.73 & 001 & NN & -4.14 & & & $\begin{array}{l}\text { Predicted } \sigma_{\mathrm{NW}}= \\
17.3 ; \sigma_{\text {tap }}=17.6\end{array}=$ \\
\hline PSZ1 G189.27+59.24 & 5.73 & 111 & $\mathrm{R}$ & & $\begin{array}{l}\text { RXC J1031.7+3502, } \\
\text { A1033 }\end{array}$ & 0.1259 & \\
\hline PSZ1 G095.37+14.42 & 5.72 & 011 & $\mathrm{R}$ & & & 0.1188 & \\
\hline PSZ1 G183.27+34.97 & 5.69 & 111 & $\mathrm{Y}$ & 9.64 & WHL J127.437+38.4651 & 0.3919 & \\
\hline PSZ1 G069.92-18.89 & 5.68 & 111 & $\mathrm{R}$ & & & 0.3076 & \\
\hline PSZ1 G156.88+13.48 & 5.67 & 111 & $\mathrm{Y}$ & 7.57 & & & \\
\hline PSZ1 G109.99+52.87 & 5.64 & 111 & $\mathrm{Y}$ & 17.90 & $\begin{array}{l}\text { RXC J1359.8+6231, } \\
\text { ZwCl 6429, } \\
\text { ZwCl 1358+6245 }\end{array}$ & 0.3259 & \\
\hline
\end{tabular}


Table A.1. continued.

\begin{tabular}{|c|c|c|c|c|c|c|c|}
\hline Cluster name & $\begin{array}{c}\text { Planck } \\
S / N\end{array}$ & $\begin{array}{c}\text { Planck } \\
\text { det. }\end{array}$ & Category & $\Delta \ln (\mathcal{Z})$ & $\begin{array}{c}\text { Previous } \\
\text { AMI }\end{array}$ & Redshift & Notes \\
\hline PSZ1 G179.13+60.14 & 5.61 & 111 & $\mathrm{R}$ & & $\begin{array}{l}\text { RXC J1040.7+3956, } \\
\text { A1068 }\end{array}$ & 0.1372 & \\
\hline PSZ1 G107.32-31.51 & 5.60 & 111 & $\mathrm{~N}$ & -2.03 & RXC J2350.5+2929 & 0.1498 & $\begin{array}{l}\text { Predicted } \sigma_{\mathrm{NW}}= \\
7.0 ; \sigma_{\text {tap }}=9.8\end{array}$ \\
\hline PSZ1 G084.62-15.86 & 5.59 & 111 & M & 1.47 & & & \\
\hline PSZ1 G145.19+32.14 & 5.58 & 001 & $\mathrm{R}$ & & $\begin{array}{l}\text { RXC J0811.1+7002, } \\
\text { A621 }\end{array}$ & 0.223 & \\
\hline PSZ1 G127.36-10.69 & 5.58 & 100 & $\mathrm{R}$ & & & & \\
\hline PSZ1 G097.93+19.46 & 5.54 & 111 & M & 1.30 & $4 \mathrm{C} 65.28$ & 0.25 & \\
\hline PSZ1 G136.62-25.05 & 5.52 & 111 & $\mathrm{LZ}$ & & $\begin{array}{l}\text { RXC J0152.7+3609, } \\
\text { A262 }\end{array}$ & 0.0163 & \\
\hline PSZ1 G094.54+51.01 & 5.52 & 011 & $\mathrm{Y}$ & 24.04 & WHL J227.050+57.9005 & 0.5392 & \\
\hline PSZ1 G123.55-10.34 & 5.51 & 111 & SE & & & 0.1 & $\begin{array}{l}\text { Lots of extended } \\
\text { emission across the } \\
\text { centre of the map }\end{array}$ \\
\hline PSZ1 G100.82+24.61 & 5.50 & 011 & $\mathrm{LZ}$ & & $\begin{array}{l}\text { RXC J1900.4+6958, } \\
\text { A2315 }\end{array}$ & 0.0877 & \\
\hline PSZ1 G103.58+24.78 & 5.48 & 011 & SE & & & 0.33 & $\begin{array}{l}30 \mathrm{mJy} \text { source at } \\
11 \text { arcmin leaves } \\
\text { substantial residu- } \\
\text { als at map centre }\end{array}$ \\
\hline PSZ1 G092.46-35.25 & 5.47 & 100 & SE & & & & $\begin{array}{l}\text { Large amounts of } \\
\text { extended emission } \\
\text { present on the map } \\
\text { after point source } \\
\text { subtraction }\end{array}$ \\
\hline PSZ1 G151.19+48.29 & 5.45 & 111 & $\mathrm{R}$ & & $\begin{array}{l}\text { RXC J1017.5+5934, } \\
\text { A959 }\end{array}$ & 0.353 & \\
\hline PSZ1 G109.88+27.94 & 5.44 & 111 & $\mathrm{Y}$ & 3.41 & & 0.4 & \\
\hline PSZ1 G134.31-06.57 & 5.44 & 011 & $\mathrm{Y}$ & 30.68 & & & \\
\hline PSZ1 G172.64+65.29 & 5.43 & 111 & $\mathrm{LZ}$ & & RXC J1111.6+4050 & 0.0794 & \\
\hline PSZ1 G101.52-29.96 & 5.43 & 111 & $\mathrm{R}$ & & & 0.227 & \\
\hline PSZ1 G168.34+69.73 & 5.42 & 011 & SE & & A1319 & 0.288 & $\begin{array}{l}\text { Many radio sources } \\
\text { close together and } \\
\text { unresolved on the } \\
\text { SA map, plus some } \\
\text { extended emission, } \\
\text { make source sub- } \\
\text { traction too difficult }\end{array}$ \\
\hline PSZ1 G134.59+53.41 & 5.42 & 011 & $\mathrm{~N}$ & -2.05 & WHL J177.705+62.3301 & 0.3452 & $\begin{array}{l}\text { Predicted } \sigma_{\mathrm{NW}}= \\
19.3 ; \sigma_{\text {tap }}=20.8\end{array}=$ \\
\hline PSZ1 G135.03+54.38 & 5.40 & 001 & SE & & WHL J178.058+61.3331 & 0.3169 & $\begin{array}{l}\text { Lots of extended } \\
\text { emission across the } \\
\text { centre of the map }\end{array}$ \\
\hline PSZ1 G106.49-10.43 & 5.40 & 110 & $\mathrm{R}$ & & & & \\
\hline PSZ1 G188.41+07.04 & 5.39 & 001 & $\mathrm{LZ}$ & & $\begin{array}{l}\text { RXC J0631.3+2500, } \\
\text { ZwCl 0628+2502 }\end{array}$ & 0.081 & \\
\hline PSZ1 G108.13-09.21 & 5.39 & 110 & $\mathrm{Y}$ & 29.88 & & & \\
\hline PSZ1 G090.82+44.13 & 5.37 & 110 & $\mathrm{~N}$ & -0.97 & $\mathrm{ZwCl} 1602+5917$ & 0.2544 & $\begin{array}{l}\text { Predicted } \sigma_{\mathrm{NW}} \\
2.4 ; \sigma_{\text {tap }}=2.2\end{array}$ \\
\hline PSZ1 G127.02+26.21 & 5.37 & 111 & M & 1.98 & & & \\
\hline PSZ1 G164.63+46.37 & 5.36 & 111 & M & 0.93 & $\begin{array}{l}\text { ZwCl 0934+5216, } \\
\text { PLCKESZ G164.61+46.38 }\end{array}$ & 0.3605 & \\
\hline PSZ1 G085.71+10.67 & 5.35 & 001 & $\mathrm{R}$ & & & & \\
\hline PSZ1 G050.46+67.54 & 5.35 & 111 & $\mathrm{~N}$ & -2.30 & $\begin{array}{l}\text { RXC J1432.4+3137, } \\
\text { A1930 }\end{array}$ & 0.1313 & $\begin{array}{l}\text { Predicted } \sigma_{\mathrm{NW}}= \\
11.1 ; \sigma_{\text {tap }}=15.1\end{array}$ \\
\hline
\end{tabular}


Table A.1. continued.

\begin{tabular}{|c|c|c|c|c|c|c|c|}
\hline Cluster name & $\begin{array}{c}\text { Planck } \\
S / N\end{array}$ & $\begin{array}{c}\text { Planck } \\
\text { det. }\end{array}$ & Category & $\Delta \ln (\mathcal{Z})$ & Previous & \multicolumn{2}{|c|}{$\begin{array}{c}\text { Redshitt Notes } \\
\text { AMI }\end{array}$} \\
\hline PSZ1 G137.51-10.01 & 5.33 & 010 & $\mathrm{R}$ & & & & \\
\hline PSZ1 G098.64+23.20 & 5.33 & 011 & $\mathrm{Y}$ & 5.57 & RXC J1910.4+6741 & 0.2471 & \\
\hline PSZ1 G060.50+26.94 & 5.33 & 110 & $\mathrm{R}$ & & RXC J1750.2+3504 & 0.1712 & \\
\hline PSZ1 G169.80+26.10 & 5.32 & 010 & $\mathrm{~N}$ & -1.46 & & & $\begin{array}{l}\text { Predicted } \sigma_{\mathrm{NW}}= \\
14.4 ; \sigma_{\text {tap }}=16.1\end{array}$ \\
\hline PSZ1 G135.12+57.90 & 5.29 & 010 & SE & & $\begin{array}{l}\text { RXC J1201.9+5802, } \\
\text { A1446 }\end{array}$ & 0.1031 & $\begin{array}{l}\text { Only observed on SA } \\
64 \mathrm{mJy} \text { source on } \\
\text { pointing centre }\end{array}$ \\
\hline PSZ1 G157.67+77.99 & 5.28 & 111 & $\mathrm{R}$ & & WHL J184.380+36.6865 & 0.3732 & \\
\hline PSZ1 G101.36+32.39 & 5.27 & 011 & $\mathrm{~N}$ & -1.60 & RXC J1727.4+7035 & 0.3059 & $\begin{array}{l}\text { Predicted } \sigma_{\mathrm{NW}}= \\
4.6 ; \sigma_{\text {tap }}=5.8\end{array}$ \\
\hline PSZ1 G121.75+51.81 & 5.26 & 111 & Y & 119.16 & $\mathrm{ZwCl} 1256+6537$ & 0.23765 & $\begin{array}{l}\text { Lots of unsubtracted } \\
\text { extended emission on } \\
\text { the maps; the cluster } \\
\text { is clearly detected, but } \\
\text { parameter estimation } \\
\text { may be unreliable }\end{array}$ \\
\hline PSZ1 G130.26-26.53 & 5.25 & 010 & SE & & $\mathrm{ZwCl} 0120+3538$ & 0.2159 & \\
\hline PSZ1 G084.85+20.63 & 5.25 & 111 & $\mathrm{Y}$ & 8.75 & & 0.29 & \\
\hline PSZ1 G149.38-36.86 & 5.25 & 111 & $\mathrm{Y}$ & 11.63 & A344 & 0.1696 & \\
\hline PSZ1 G138.11+42.03 & 5.24 & 011 & $\mathrm{R}$ & & & 0.4961 & \\
\hline PSZ1 G198.50+46.01 & 5.24 & 111 & M & 1.01 & $\mathrm{ZwCl} 0928+2904$ & 0.222 & \\
\hline PSZ1 G091.81-26.97 & 5.23 & 011 & $\mathrm{R}$ & & RXC J2245.4+2808 & 0.3551 & \\
\hline PSZ1 G031.91+67.94 & 5.23 & 100 & $\mathrm{~N}$ & -0.29 & & & $\begin{array}{l}\text { Predicted } \sigma_{\mathrm{NW}} \\
5.8 ; \sigma_{\text {tap }}=6.5\end{array}$ \\
\hline PSZ1 G213.37+80.60 & 5.23 & 111 & $\mathrm{Y}$ & 22.50 & WHL J182.349+26.6796 & 0.5586 & \\
\hline PSZ1 G100.03+23.73 & 5.22 & 001 & Y & 8.84 & $\begin{array}{l}\text { RXC J1908.3+6903, } \\
\text { A2317 }\end{array}$ & 0.2103 & \\
\hline PSZ1 G135.92+76.21 & 5.22 & 010 & $\mathrm{~N}$ & -2.91 & & & $\begin{array}{l}\text { Predicted } \sigma_{\mathrm{NW}} \\
2.6 ; \sigma_{\text {tap }}=4.0\end{array}$ \\
\hline PSZ1 G071.44+59.57 & 5.21 & 111 & $\mathrm{R}$ & & $\begin{array}{l}\text { RXC J1501.3+4220, } \\
\text { ZwCl 7215, } \\
\text { ZwCl 1459+4240 }\end{array}$ & 0.2917 & \\
\hline PSZ1 G164.26+08.91 & 5.21 & 111 & $\mathrm{Y}$ & 14.38 & WHL J85.8665+46.9358 & 0.2505 & \\
\hline PSZ1 G084.84+35.04 & 5.21 & 111 & $\mathrm{~N}$ & -0.66 & $\begin{array}{l}\text { RXC J1718.1+5639, } \\
\text { ZwCl } 8197\end{array}$ & 0.1138 & $\begin{array}{l}\text { Predicted } \sigma_{\mathrm{NW}}= \\
5.0 ; \sigma_{\text {tap }}=5.3\end{array}$ \\
\hline PSZ1 G119.37+46.84 & 5.21 & 111 & SE & & $\begin{array}{l}\text { RXC J1320.0+7003, } \\
\text { A1722, } \\
\text { MCS J1319.9+7003 }\end{array}$ & 0.3275 & $\begin{array}{l}\text { Extended structure to } \\
\text { the west not detected } \\
\text { in LA map }\end{array}$ \\
\hline PSZ1 G076.44+23.53 & 5.21 & 111 & SE & & & 0.1685 & \\
\hline PSZ1 G077.71+26.72 & 5.20 & 011 & LZ & & $\begin{array}{l}\text { RXC J1811.0+4954, } \\
\text { ZwCl } 8338\end{array}$ & 0.0501 & \\
\hline PSZ1 G183.26+12.25 & 5.20 & 011 & $\mathrm{~N}$ & -1.52 & & & $\begin{array}{l}\text { Predicted } \sigma_{\mathrm{NW}} \\
17.1 ; \sigma_{\text {tap }}=18.2\end{array}$ \\
\hline PSZ1 G085.85+35.45 & 5.20 & 011 & LZ & & RXC J1715.3+5724 & 0.0276 & \\
\hline PSZ1 G114.98+19.10 & 5.19 & 010 & $\mathrm{~N}$ & -0.75 & & & $\begin{array}{l}\text { Predicted } \sigma_{\mathrm{NW}} \\
14.6 ; \sigma_{\text {tap }}=17.0\end{array}$ \\
\hline PSZ1 G059.51+33.06 & 5.18 & 011 & SE & & $\begin{array}{l}\text { RXC J1720.2+3536, } \\
\text { MCS J1720.2+3536 }\end{array}$ & 0.387 & $\begin{array}{l}280 \mathrm{mJy} \text { source at } 13 \\
\text { arcmin produces arti- } \\
\text { facts on SA map }\end{array}$ \\
\hline PSZ1 G172.93+21.31 & 5.18 & 011 & $\mathrm{Y}$ & 4.86 & & 0.3309 & \\
\hline PSZ1 G091.93+35.48 & 5.18 & 100 & $\mathrm{~N}$ & -2.80 & & & $\begin{array}{l}\text { Predicted } \sigma_{\mathrm{NW}} \\
14.1 ; \sigma_{\text {tap }}=12.4\end{array}$ \\
\hline PSZ1 G075.29+26.66 & 5.17 & 100 & $\mathrm{~N}$ & -2.85 & & & $\begin{array}{l}\text { Predicted } \sigma_{\mathrm{NW}} \\
17.3 ; \sigma_{\text {tap }}=17.1\end{array}$ \\
\hline PSZ1 G175.89+24.24 & 5.16 & 010 & $\mathrm{~N}$ & -0.65 & $\mathrm{ZwCl} 0723+4239$ & 0.19175 & $\begin{array}{l}\text { Predicted } \sigma_{\mathrm{NW}} \\
2.7 ; \sigma_{\text {tap }}=2.5\end{array}$ \\
\hline
\end{tabular}


Table A.1. continued.

\begin{tabular}{|c|c|c|c|c|c|c|c|}
\hline Cluster name & $\begin{array}{c}\text { Planck } \\
S / N \\
\end{array}$ & $\begin{array}{c}\text { Planck } \\
\text { det. }\end{array}$ & Category & $\Delta \ln (\mathcal{Z})$ & $\begin{array}{c}\text { Previous } \\
\text { AMI }\end{array}$ & Redshift & Notes \\
\hline PSZ1 G144.86+25.09 & 5.15 & 111 & $\mathrm{Y}$ & 44.36 & $\begin{array}{l}\text { RXC J0647.8+7014, } \\
\text { MCS J0647.6+7015 }\end{array}$ & 0.584 & \\
\hline PSZ1 G123.72+34.65 & 5.14 & 100 & $\mathrm{R}$ & & RXC J1231.3+8225 & 0.2053 & \\
\hline PSZ1 G197.13+33.46 & 5.13 & 110 & $\mathrm{R}$ & & WHL J128.694+26.9757 & 0.4561 & \\
\hline PSZ1 G122.98-35.52 & 5.11 & 001 & $\mathrm{Y}$ & 11.74 & RXC J0051.6+2720 & 0.3615 & \\
\hline PSZ1 G053.50+09.56 & 5.11 & 101 & $\mathrm{NN}$ & -4.20 & & & $\begin{array}{l}\text { Predicted } \sigma_{\mathrm{NW}}= \\
15.9 ; \sigma_{\text {tap }}=19.3\end{array}$ \\
\hline PSZ1 G045.07+67.80 & 5.11 & 100 & $\mathrm{~N}$ & -2.05 & A1929 & 0.2191 & $\begin{array}{l}\text { Predicted } \begin{array}{l}\sigma_{\mathrm{NW}} \\
13.8 ; \sigma_{\text {tap }}=13.4\end{array}=\end{array}$ \\
\hline PSZ1 G116.79-09.82 & 5.11 & 011 & $\mathrm{R}$ & & $\mathrm{ZwCl} 0008+5215$ & 0.104 & \\
\hline PSZ1 G189.29+07.44 & 5.10 & 001 & $\mathrm{R}$ & & & & \\
\hline PSZ1 G103.16-14.95 & 5.08 & 110 & SE & & & & \\
\hline PSZ1 G157.84+21.23 & 5.08 & 111 & M & 2.15 & & & \\
\hline PSZ1 G048.09+27.18 & 5.07 & 111 & M & 1.04 & & 0.73608 & \\
\hline PSZ1 G087.47+37.65 & 5.07 & 010 & $\mathrm{R}$ & & & 0.1132 & \\
\hline PSZ1 G111.74+70.35 & 5.07 & 111 & M & 1.06 & $\begin{array}{l}\text { RXC J1313.1+4616, } \\
\text { A1697 }\end{array}$ & 0.183 & \\
\hline PSZ1 G066.20+12.87 & 5.06 & 001 & $\mathrm{~N}$ & -0.95 & & 0.23 & $\begin{array}{l}\text { Predicted } \sigma_{\mathrm{NW}}= \\
9.3 ; \sigma_{\text {tap }}=11.2\end{array}$ \\
\hline PSZ1 G045.85+57.71 & 5.06 & 111 & $\mathrm{Y}$ & 10.15 & & 0.611 & \\
\hline PSZ1 G079.33+28.33 & 5.06 & 011 & SE & & $\mathrm{ZwCl} 1801+5136$ & 0.2036 & $\begin{array}{l}\text { Too many radio } \\
\text { sources near the clus- } \\
\text { ter centre to be sure of } \\
\text { a non-detection }\end{array}$ \\
\hline PSZ1 G097.52-14.92 & 5.06 & 010 & $\mathrm{Y}$ & 35.39 & & & $\begin{array}{l}\text { Bright, extended ra- } \\
\text { dio galaxy at about } \\
10 \text { arcmin removed } \\
\text { from the SA data man- } \\
\text { ually using CLEAN } \\
\text { components leaving } \\
\text { significant residuals in } \\
\text { the source-subtracted } \\
\text { map; cluster is clearly } \\
\text { detected but parameter } \\
\text { estimation is suspect }\end{array}$ \\
\hline PSZ1 G118.06+31.10 & 5.05 & 011 & SE & & & & $\begin{array}{l}\text { Extended emission } \\
\text { near the cluster centre }\end{array}$ \\
\hline PSZ1 G056.13+28.06 & 5.05 & 011 & $\mathrm{Y}$ & 4.13 & WHL J265.066+31.6026 & 0.426 & \\
\hline PSZ1 G083.35+76.41 & 5.03 & 011 & $\mathrm{R}$ & & & & \\
\hline PSZ1 G073.64+36.49 & 5.03 & 001 & $\mathrm{~N}$ & -0.05 & & 0.56 & $\begin{array}{l}\text { Predicted } \begin{array}{l}\sigma_{\mathrm{NW}} \\
21.1 ; \sigma_{\text {tap }}=\end{array}=22.2\end{array}=$ \\
\hline PSZ1 G129.81+16.85 & 5.03 & 100 & $\mathrm{Y}$ & 3.91 & & 0.41159 & \\
\hline PSZ1 G134.64-11.77 & 5.02 & 111 & $\mathrm{Y}$ & 31.69 & & & $\begin{array}{l}66 \mathrm{mJy} \text { source at } \\
10 \text { arcmin leaves } \\
\text { residuals in the source- } \\
\text { subtracted map; cluster } \\
\text { is clearly detected bu } \\
\text { parameter estimation } \\
\text { is suspect }\end{array}$ \\
\hline PSZ1 G178.10+18.58 & 5.01 & 101 & SE & & & & \\
\hline PSZ1 G165.41+66.17 & 5.00 & 111 & M & 1.96 & WHL J170.907+43.0578 & 0.1957 & \\
\hline PSZ1 G099.48+37.72 & 5.00 & 101 & M & 0.79 & $\begin{array}{l}\text { RXC J1634.6+6738, } \\
\text { A2216 }\end{array}$ & 0.1668 & \\
\hline
\end{tabular}


Y. C. Perrott et al.: Planck and AMI SZ measurements for 99 galaxy clusters

\section{Appendix B: $Y_{\text {tot }}-\theta_{\mathrm{s}}$ posterior comparison}
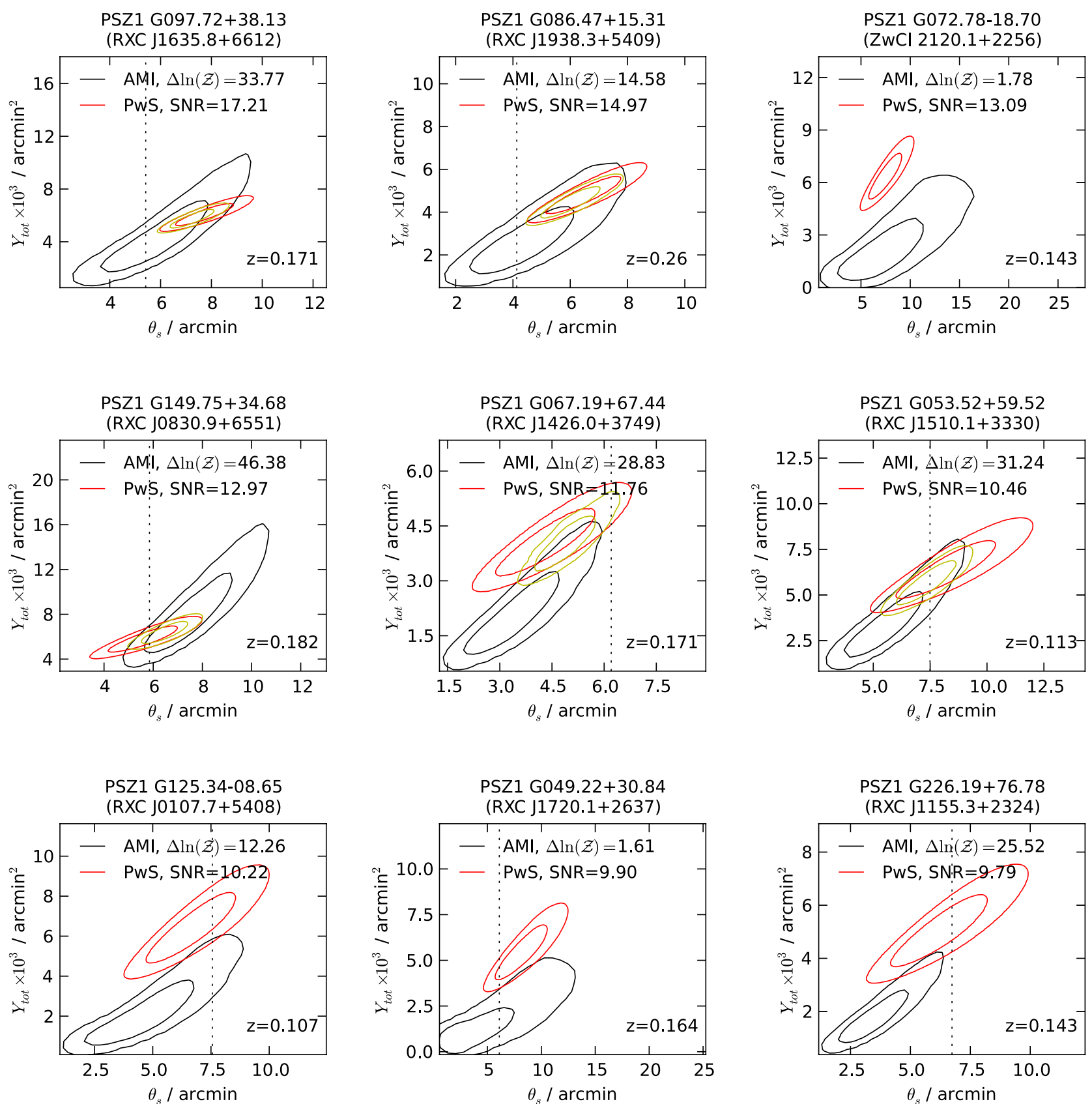

Fig. B.1. $Y_{\text {tot }}-\theta_{\mathrm{s}}$ posterior distributions for AMI and Planck, in descending Planck $\mathrm{S} / \mathrm{N}$ order (note that this is the compatibility $\mathrm{S} / \mathrm{N}$ for PWS), for all AMI detections $(\Delta \ln (\mathcal{Z}) \geq 0$ ). Contours mark the $68 \%$ and $95 \%$ confidence limits of the posterior distributions. Where available, $X$-ray values for $\theta_{\mathrm{s}}$ converted from $\theta_{500}$ values from Piffaretti et al. (2011) using the universal $c_{500}=1.177$ are shown with black dotted lines. 
A\&A 580, A95 (2015)
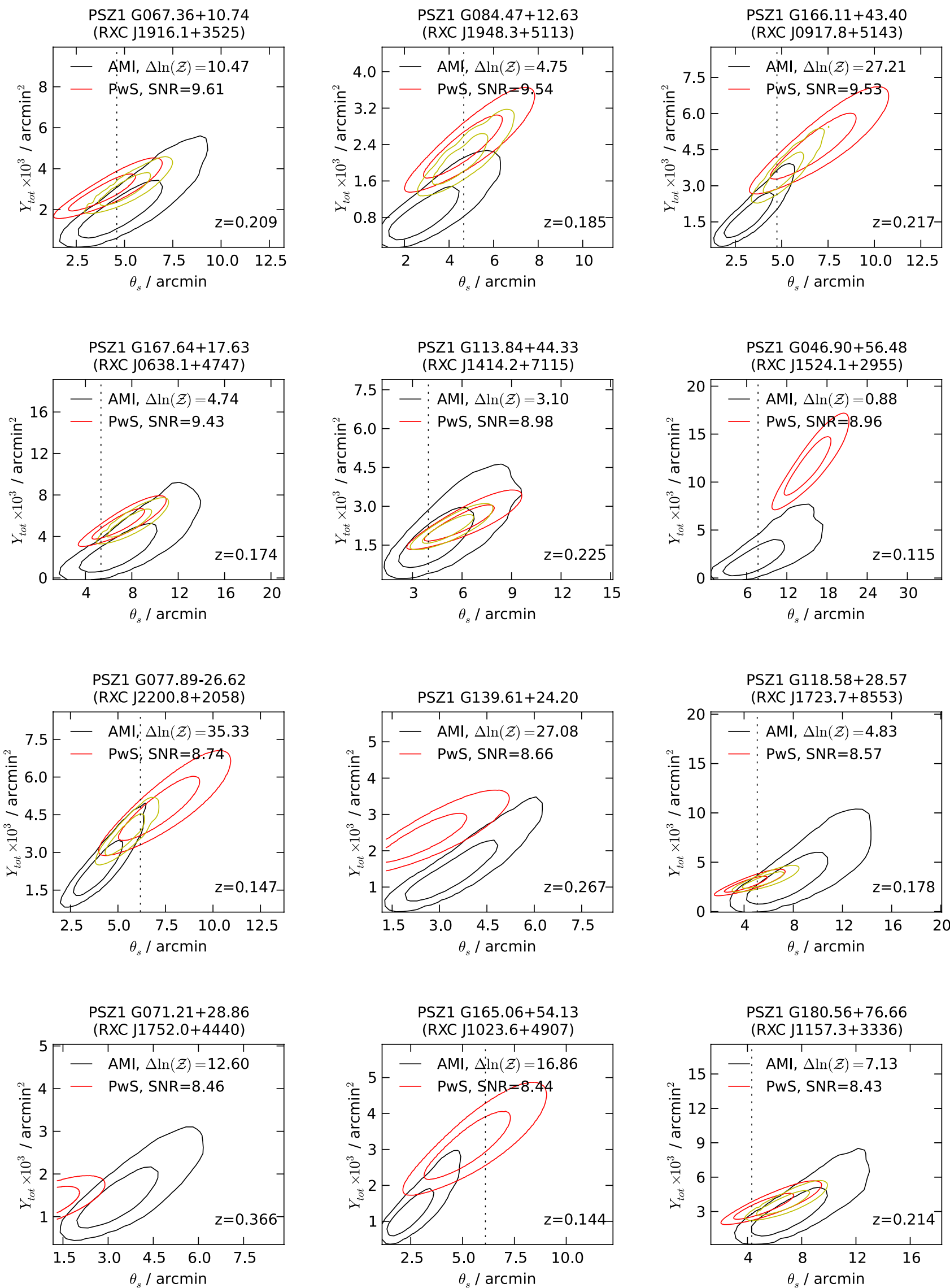

Fig. B.1. continued. 
Y. C. Perrott et al.: Planck and AMI SZ measurements for 99 galaxy clusters
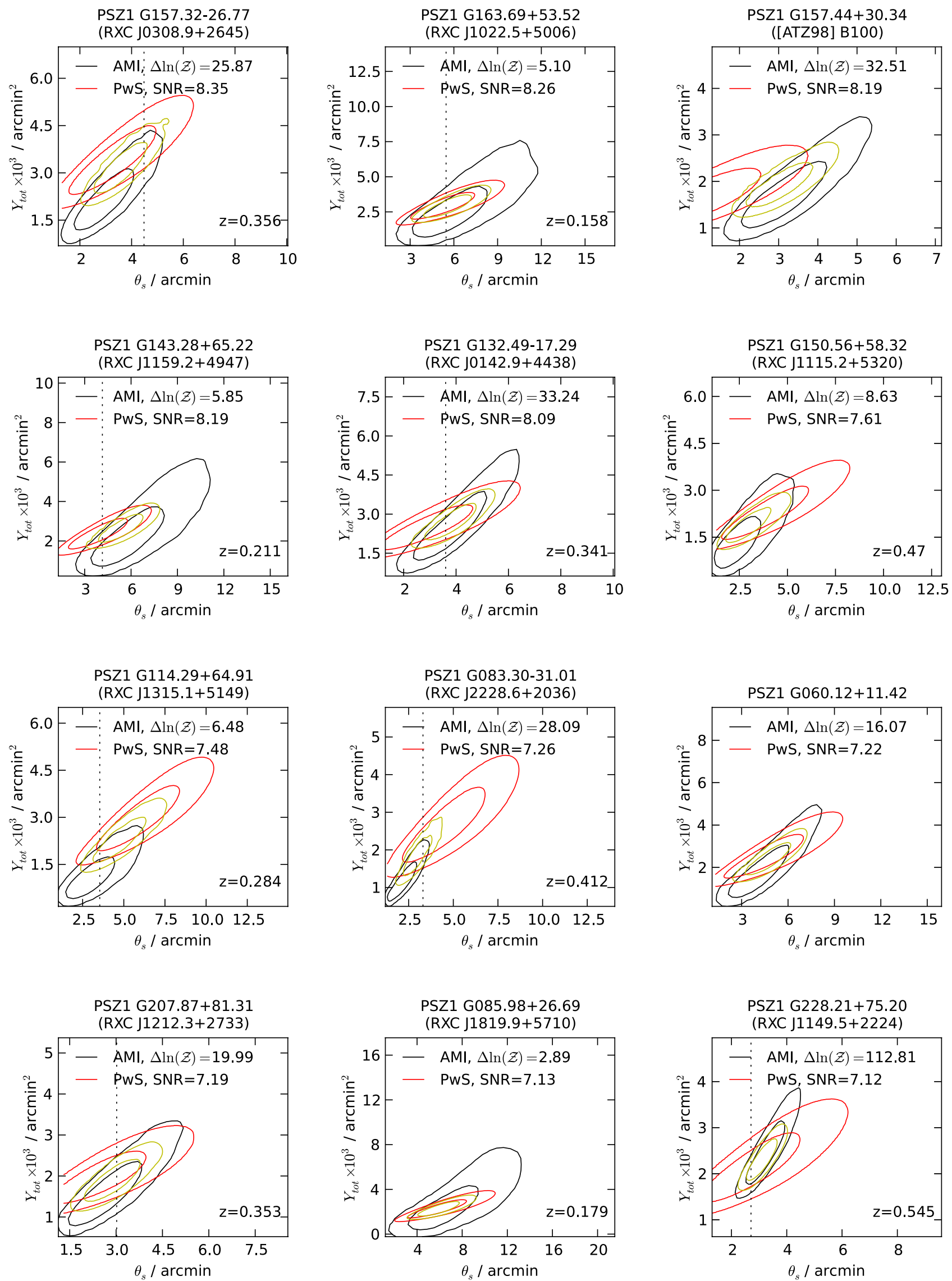

Fig. B.1. continued. 

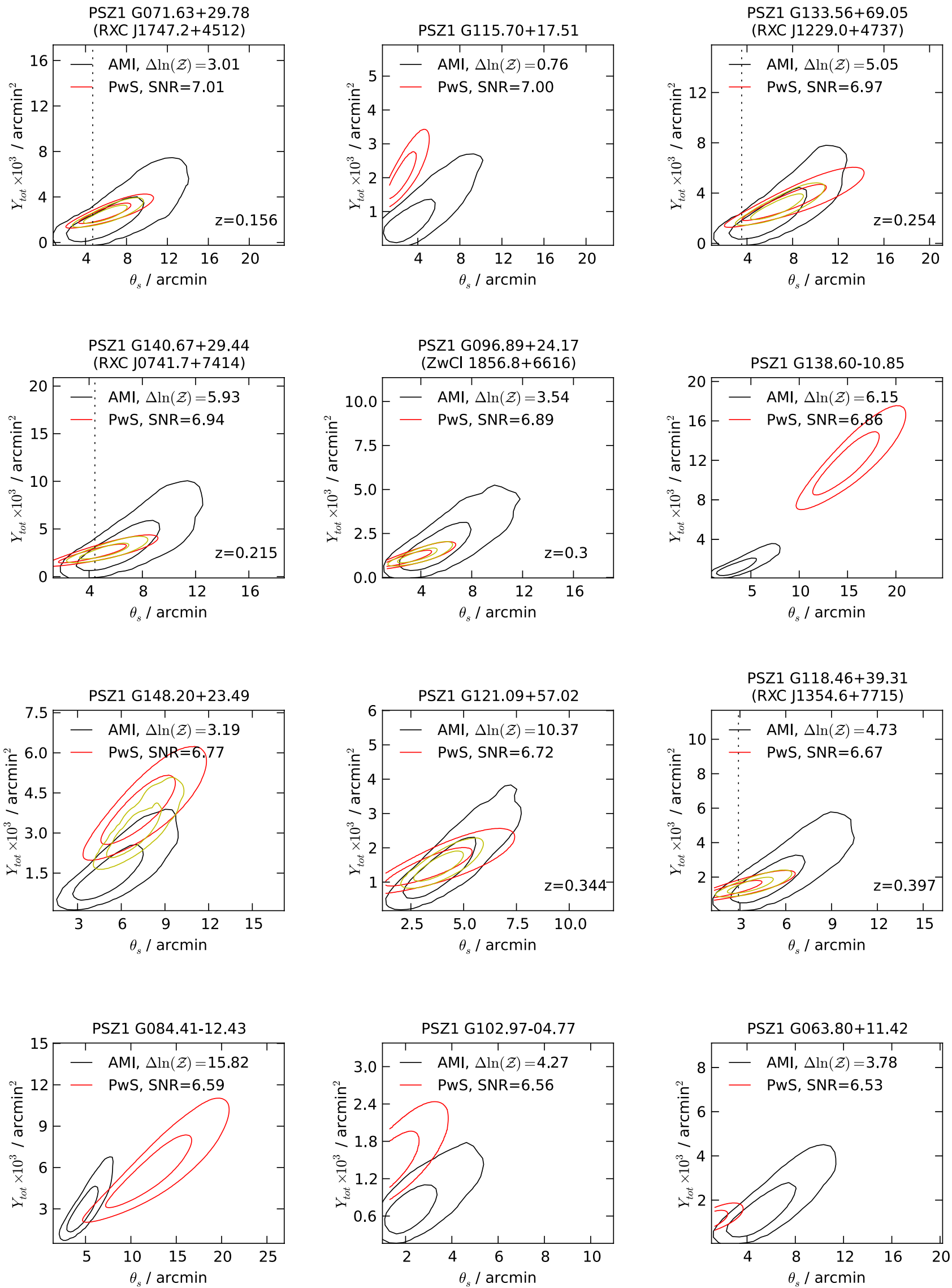

Fig. B.1. continued. 
Y. C. Perrott et al.: Planck and AMI SZ measurements for 99 galaxy clusters
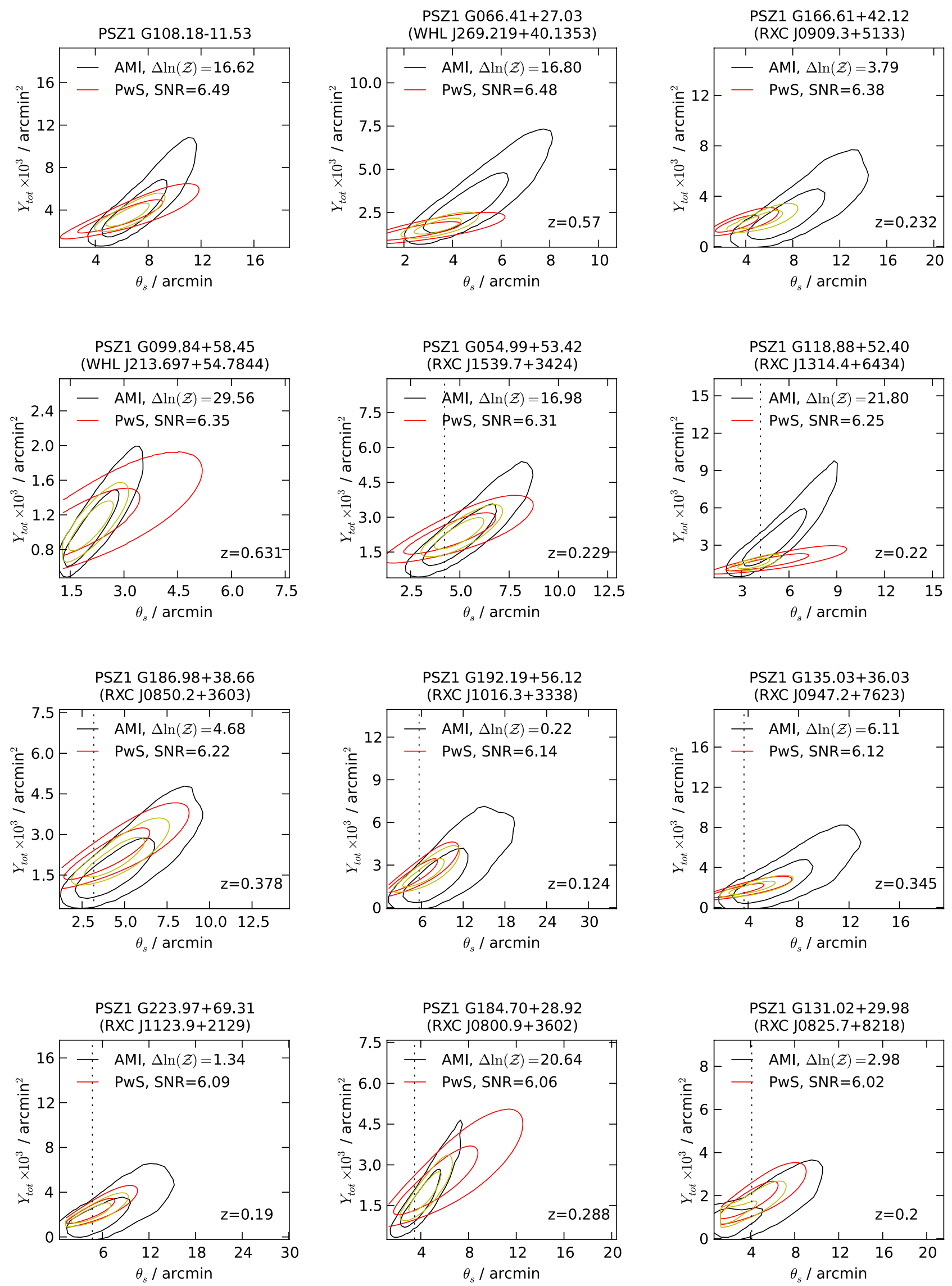

Fig. B.1. continued. 

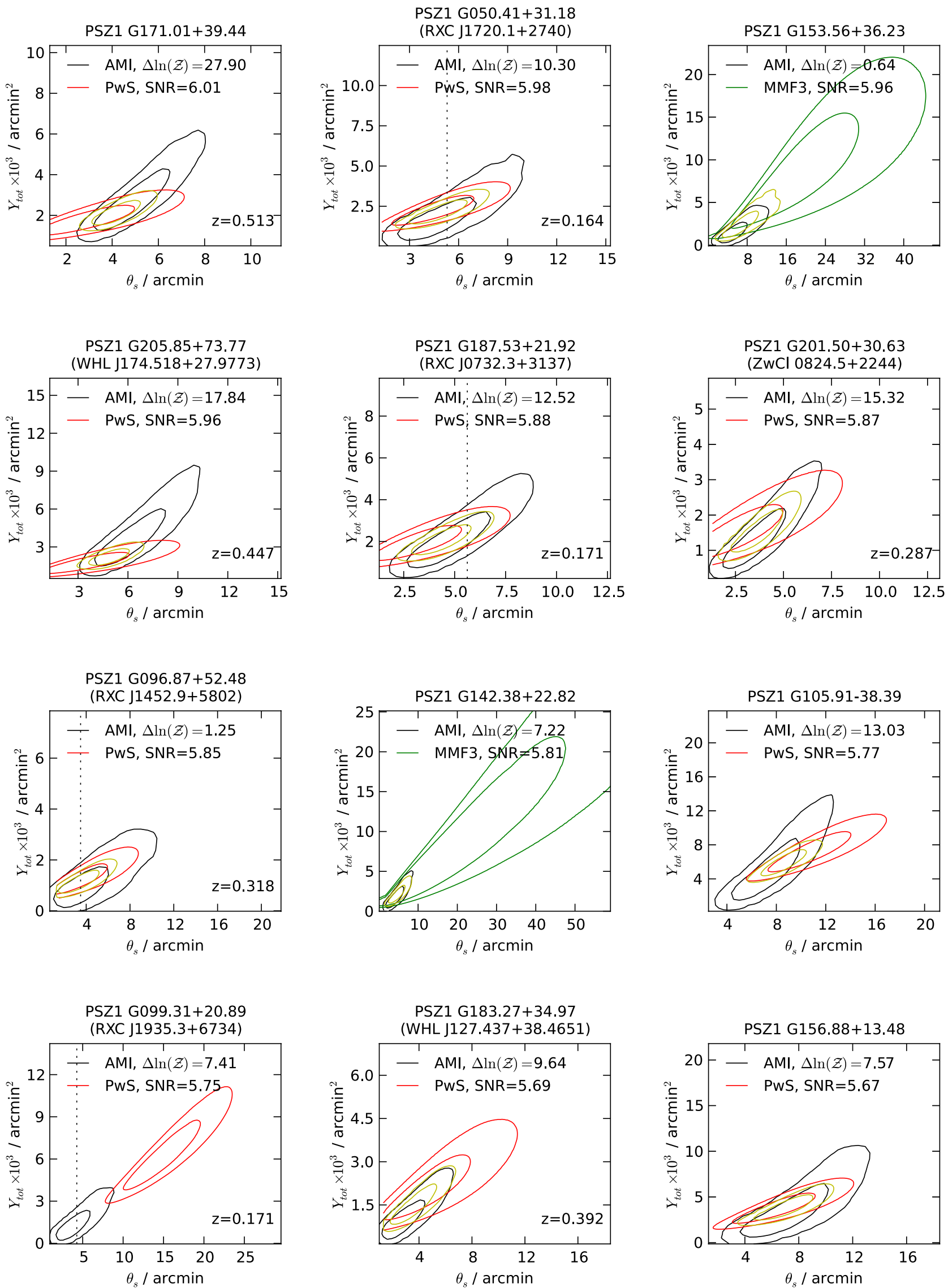

Fig. B.1. continued. 
Y. C. Perrott et al.: Planck and AMI SZ measurements for 99 galaxy clusters
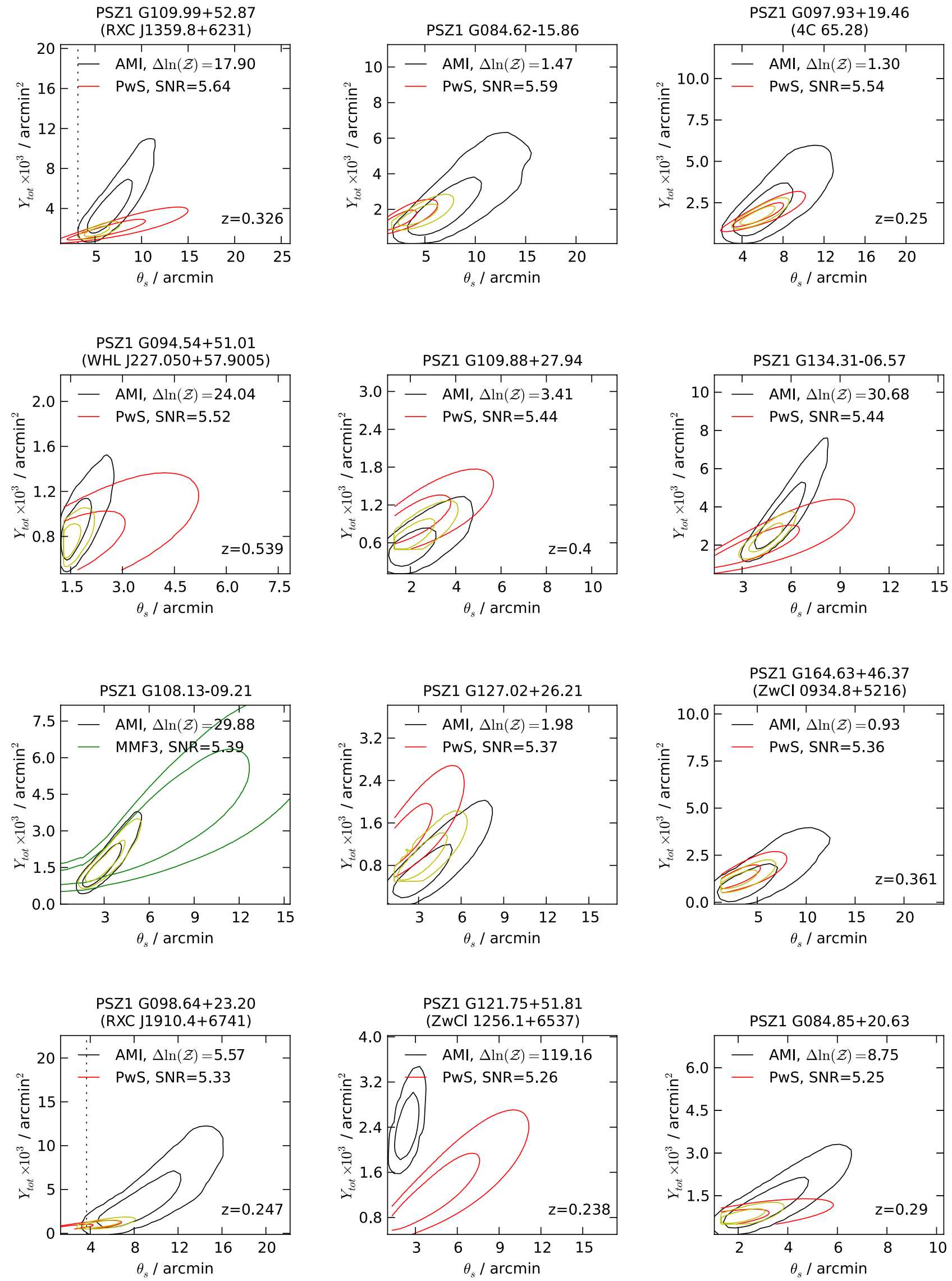

Fig. B.1. continued. 
A\&A 580, A95 (2015)
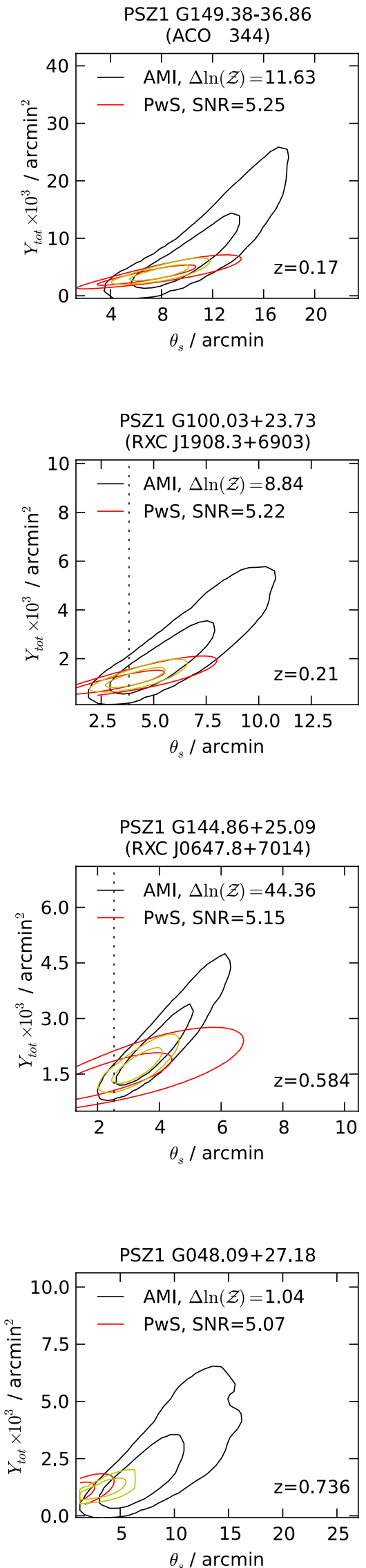

Fig. B.1. continued.
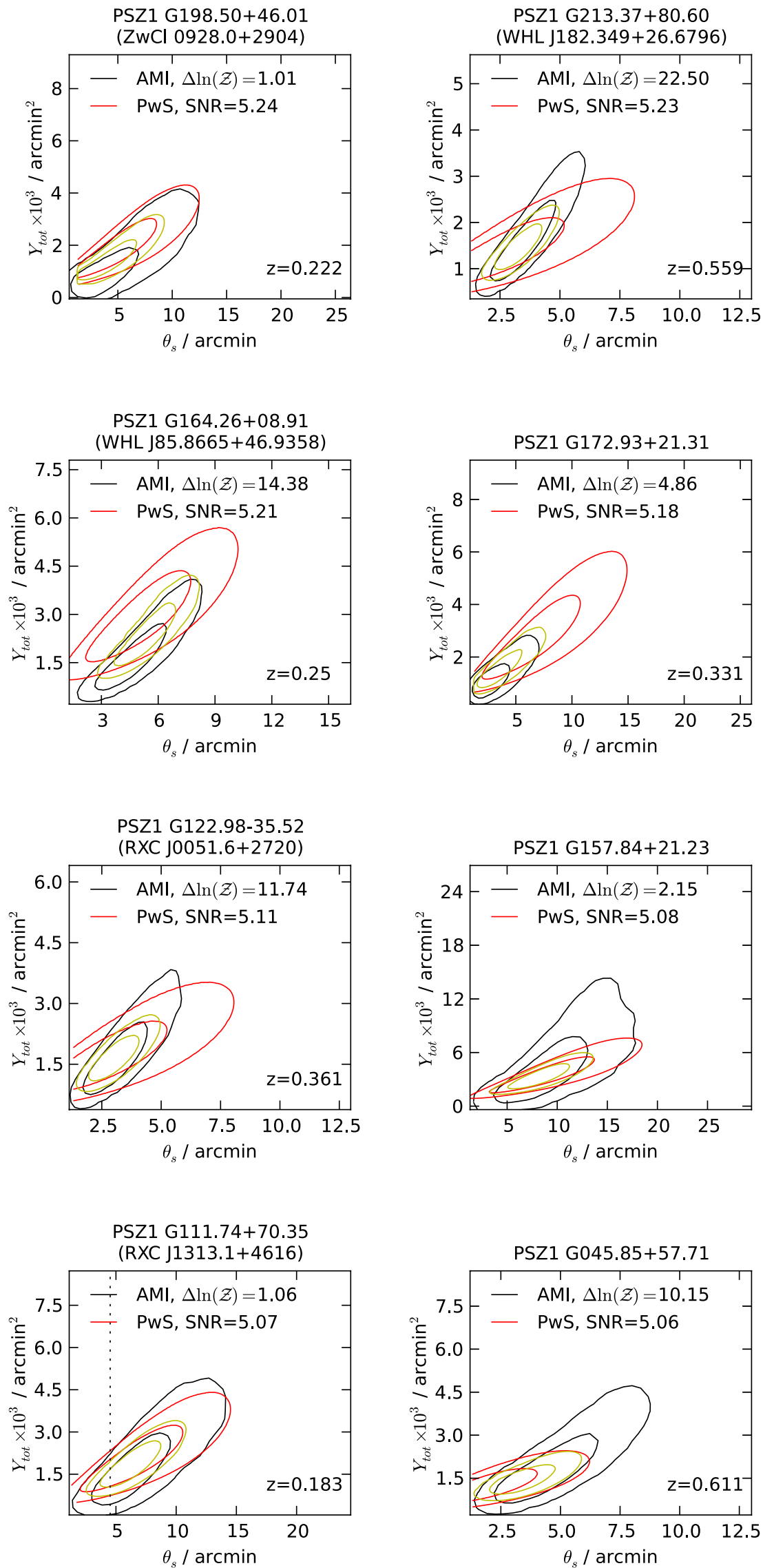
Y. C. Perrott et al.: Planck and AMI SZ measurements for 99 galaxy clusters
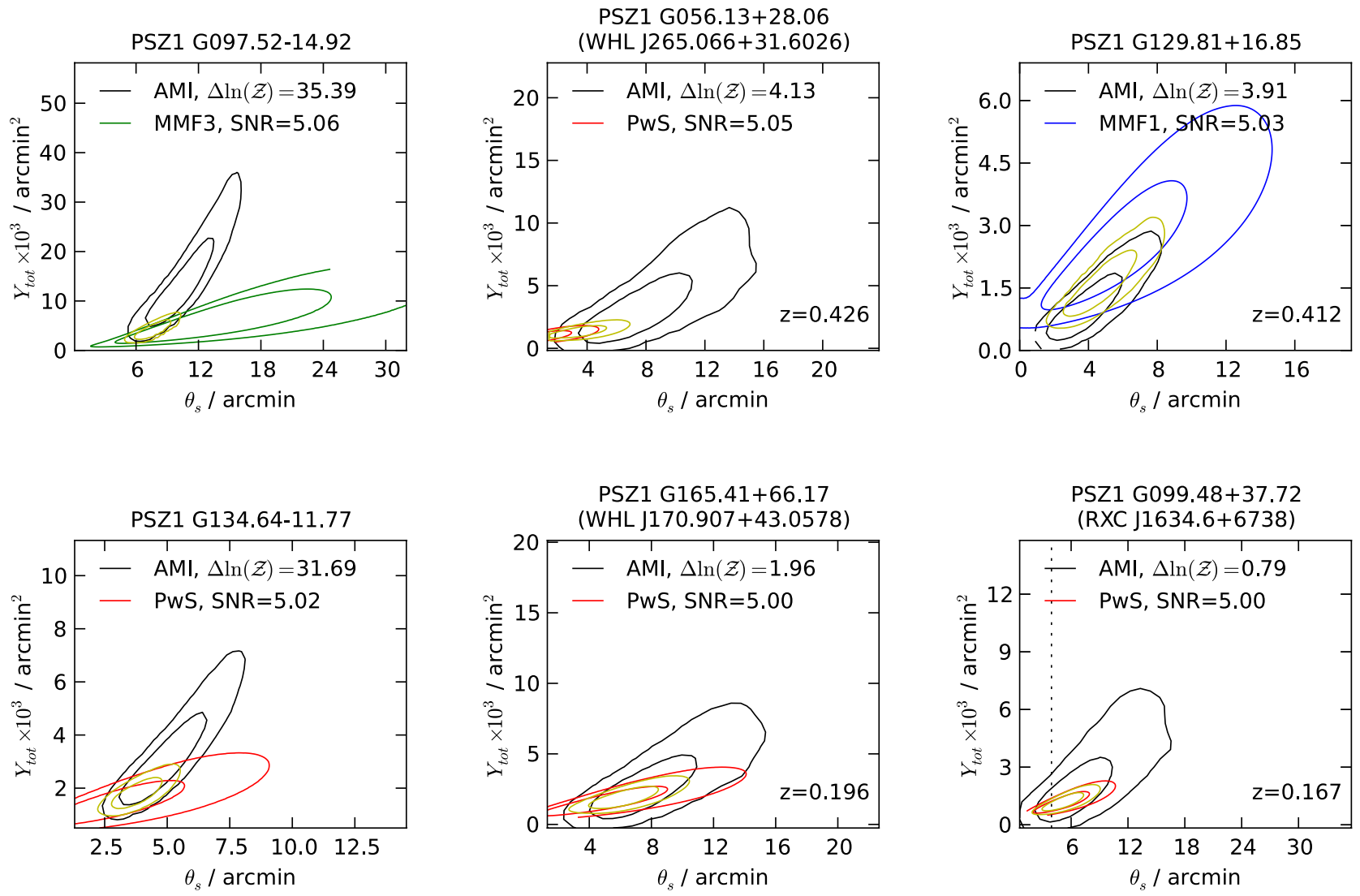

Fig. B.1. continued. 

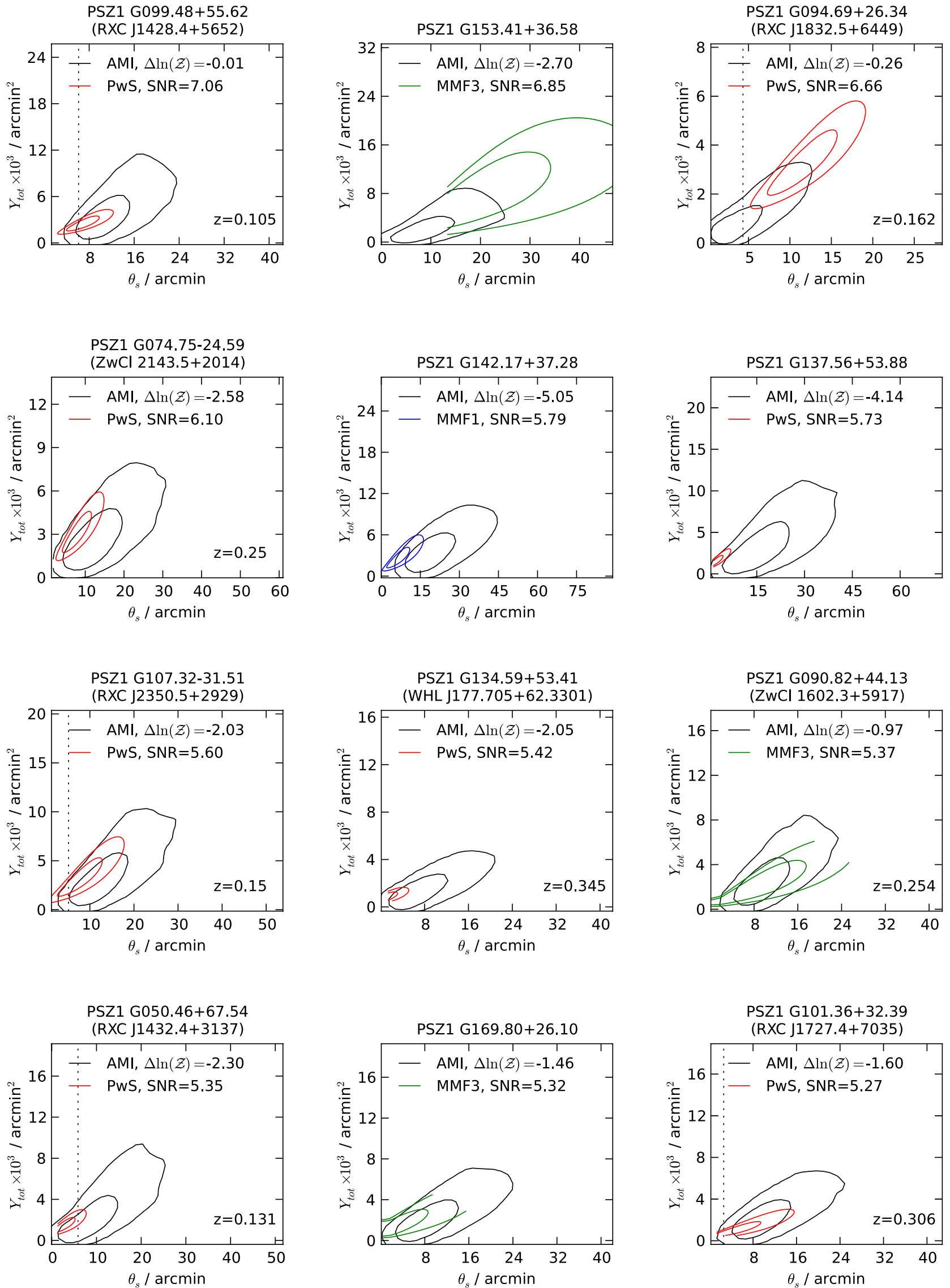

Fig. B.2. $Y_{\text {tot }}-\theta_{\mathrm{s}}$ posterior distributions for AMI and Planck, in descending Planck $\mathrm{S} / \mathrm{N}$ order (note that this is the compatibility $\mathrm{S} / \mathrm{N}$ for PWS), for all AMI non-detections $(\Delta \ln (\mathcal{Z})<0)$. Contours mark the $68 \%$ and $95 \%$ confidence limits of the posterior distributions. Where available, X-ray values for $\theta_{\mathrm{s}}$ converted from $\theta_{500}$ values from Piffaretti et al. (2011) using the universal $c_{500}=1.177$ are shown with black dotted lines. 
Y. C. Perrott et al.: Planck and AMI SZ measurements for 99 galaxy clusters
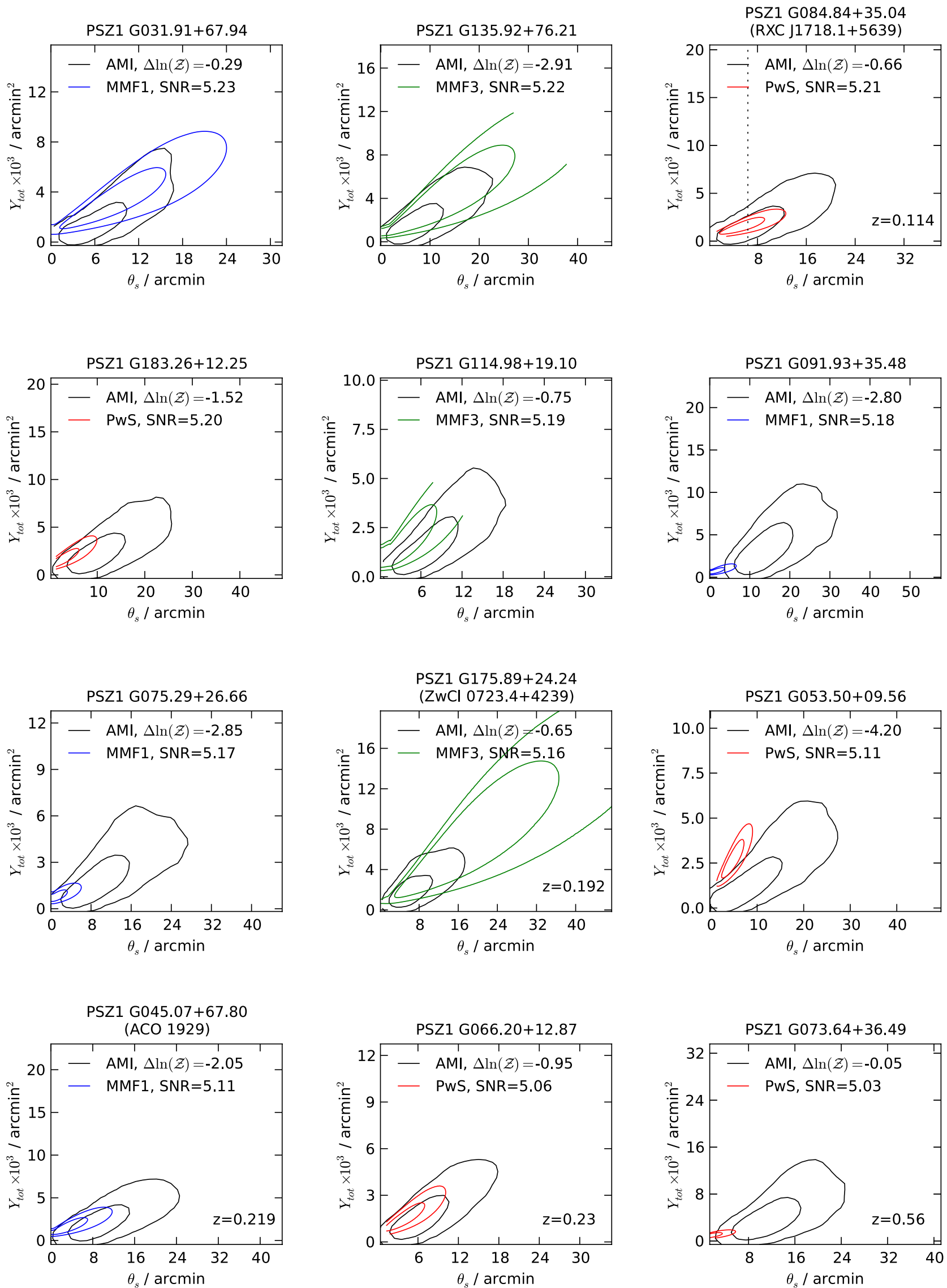

Fig. B.2. continued. 